\title{
Functional food science and defence against reactive oxidative species
}

\author{
A. T. Diplock ${ }^{1} *$ J.-L. Charleux ${ }^{2}$, G. Crozier-Willi ${ }^{3}$, F. J. Kok ${ }^{4}$, C. Rice-Evans ${ }^{1}$, M. Roberfroid ${ }^{5}$, \\ W. Stahl ${ }^{6}$ and J. Viña-Ribes ${ }^{7}$ \\ ${ }^{1}$ International Antioxidant Research Centre, UMDS, Guy's Hospital, St Thomas Street, London SE1 9RT, UK \\ ${ }^{2}$ F. Hoffmann-La Roche Ltd, Business Unit Carotenoids, Headoffice Kaiseraugst VMI Building, CH04002 Basel, Switzerland \\ ${ }^{3}$ Nestec Ltd, Nestlé Research Center, Vers-Chez-Les-Blanc, PO Box 44, CH-1000 Lausanne 26, Switzerland \\ ${ }^{4}$ Division of Human Nutrition and Epidemiology, Wageningen Agricultural University, PO Box 8129, NL 6700 EV, \\ Wageningen, The Netherlands \\ ${ }^{5}$ UCL, Ecole de Pharmacie, Tour Van Helmont, Avenue E. Mounier, B-1200 Brussels, Belgium \\ ${ }^{6}$ Heinrich-Heine-University Düsseldorf, Medizinische Einrichtungen, Institut für Physiologische Chemie I, Postfach 101007 , \\ D-40001 Düsseldorf, Germany \\ ${ }^{7}$ Universidad de Valencia, Facultad de Medicina, Departamento de Fisiologia, Avenida Blasco Ibafiez 17, E-46010 Valencia, Spain
}

\section{Contents}

1. Introduction

2. Oxidative damage, antioxidant defence and the role of prooxidants in disease

2.1. Antioxidant defence system of the human organism

2.1.1 Origins and nature of free radicals and other oxidants

2.1.2. Enzymic and non-enzymic defence systems in vivo

2.1.3. Dietary antioxidants; nutrient and nonnutrient

2.2. Oxidative damage to bodily functions and its implications in disease

2.2.1. Coronary heart disease

2.2.2. Carcinogenesis

2.2.3. Cataract and age-related macular degeneration

2.2.4. Neuronal diseases

2.3. Conclusions

3. Available methodologies for evaluating and quantifying ex vivo damage to DNA, lipids and proteins by prooxidants in vivo

3.1. Oxidative damage to DNA

3.1.1. Measurement of guanine damage products in DNA by HPLC and gas chromatographymass spectrometry ( $G C-M S)$

3.2. Oxidative damage to lipids

3.2.1 Lipid peroxidation

3.2.2. Can some measure of 'total' peroxidation be obtained?

3.2.3. LDL oxidation

3.3. Oxidative damage to proteins

3.4. Measurement of antioxidant nutrients, carotenoids and flavonoids extracted from human plasma
S79

$\mathrm{S} 80$

$\mathrm{S} 80$

S80

S80

S80

S82

S82

S83

S83

S83

S84

S84

S84

S84

S85

S85

S86

S86

S87

S87
3.5. Conclusions

S88

4. Nutritional options modulating oxidative damage and antioxidant defence systems

4.1. Introduction

4.2. Dietary antioxidants

4.2.1. Sources of dietary antioxidants

4.2.2. Antioxidant intake and status

4.2.3. Bioavailability of antioxidants

4.2.4. Fat intake and antioxidant status

4.3. Epidemiological studies on protective effects of antioxidants

4.3.1. Cardiovascular disease

S90

4.3.2. Cancer

4.3.3. Other age-related diseases

S90

S91

S91

4.4. Human intervention studies of antioxidants $\quad \mathrm{S} 92$

4.4.1. Cardiovascular disease

S92

4.4.2. Cancer

S92

4.5. Conclusions

5. Potential safety implications related to antioxidant

nutritional enhancement

S93

5.1. Introduction

S93

5.2. Vitamin $C$

5.3. Vitamin $E$

5.4. Carotenoids

5.5. Non-nutrient antioxidants (flavonoids and other related compounds)

5.5.1. Absorption

5.5.2. Possible adverse effects

S93

\$93

S94

S96

S96

S96

S97

6. Role of food technology in nutritional and safety aspects of antioxidants

S97

6.1. Introduction $\quad \mathrm{S} 97$

6.2. Physical processes

\footnotetext{
Abbreviations: AMD, age-related macular degeneration; ATBC study, $\alpha$-tocopherol $\beta$-carotene study; FOX method, ferrous oxidation in xylenol orange method; GC-MS, gas chromatography-mass spectrometry; 8-OHdG, 8-hydroxy-deoxyguanosine; 8-OHG, 8-hydroxyguanosine; 8-oxodG, 8-oxo-7,8dihydro-2'-deoxyguanine; PG, prostaglandin; PUFA, polyunsaturated fatty acids; RDA, recommended daily allowance; RNS, reactive nitrogen species; ROS, reactive oxygen species; TBA, thiobarbituric acid; TBARS, TBA-reactive substances; $\alpha$-TE, $\alpha$-tocopherol equivalents.

* Corresponding author: Professor A. T. Diplock, fax +44 (0) 171403 7195, tel +44 (0) 1719554521.
} 
6.2.1. Structural integrity $\quad \mathrm{S} 98$

6.2.2. Moisture content $\quad \mathrm{S} 98$

6.2.3. Temperature $\quad \mathrm{S} 98$

6.2.4. Minimizing oxygen $\quad 599$

6.2.5. Protection from light $\quad \mathrm{S} 99$

6.2.6. Irradiation $\quad$ S99

6.3. Chemical processes $\quad \$ 99$

6.3.1. Enzymes $\quad \$ 99$

6.3.2. Supplementation $\quad$ S99

6.4. Conclusions $\$ 100$

7. Critical assessment of the science base and
conclusions

$\begin{array}{ll}\text { 7.1. Identification of criteria } & \mathrm{S} 100\end{array}$

7.2. Critical evaluation of the present knowledge base $\mathrm{S} 101$

7.2.1. Conclusions from section $2 \quad \mathrm{~S} 101$

7.2.2. Conclusions from section $3 \quad \mathrm{~S} 101$

7.2.3. Conclusions from section $4 \quad \mathrm{~S} 102$
7.2.4. Conclusions from section $5 \quad \mathrm{~S} 102$

7.2.5. Conclusions from section $6 \quad \mathrm{~S} 102$

7.3. Evaluation of criteria $\quad \mathrm{S} 102$

7.4. Final conclusions $\quad \mathrm{S} 103$

8. Recommendations for future research S104

8.1. Introduction $\mathrm{S} 104$

8.2. Specific recommendations $\mathrm{S} 104$

8.2.1. Oxidative damage and antioxidant defence systems of the human organism

S104

8.2.2. Ex vivo methodologies for quantitating and validating damage in vivo to biological macromolecules

S104

8.2.3. Nutritional options modulating oxidative damage

8.2.4. Safety implications of nutritional enhancement of antioxidants

8.3. Priorities for the recommendations made

\section{Abstract}

This paper assesses critically the science base that underpins the argument that oxidative damage is a significant causative factor in the development of human diseases and that antioxidants are capable of preventing or ameliorating these disease processes. The assessment has been carried out under a number of headings, and some recommendations for future research are made based on the present day knowledge base.

\section{The knowledge database}

(1) Consideration of the basic science that underlies understanding of the role of free radicals in causing cellular pathologies, and the role of antioxidants in preventing this, shows that an imbalance of reactive oxygen species and antioxidant defence systems may lead to chemical modifications of biologically relevant macromolecules. This imbalance provides a logical pathobiochemical mechanism for the initiation and development of several disease states. Experimental data obtained in vivo provide evidence that antioxidants function in systems that scavenge reactive oxygen species and that these are relevant to what occurs in vivo. The relevance in vivo of these observations depends inter alia on knowledge of the uptake and distribution of the antioxidant within the human body, and on what tissue levels of the antioxidant may be expected in relation to dietary levels.

(2) There is some way to go until validated precise methods are available for measuring biomarkers of oxidative damage in human subjects in vivo under minimally invasive conditions. With respect to oxidative damage to DNA, HPLC and GC-mass spectrophotometry methods have both merits and limitations. Lipid oxidation products in plasma are best measured as isoprostanes or as lipid hydroperoxides using specific HPLC techniques. Development of isoprostane measurement will advance specificity and precision. The measurement of oxidative damage to proteins has some potential but such methods have not been effectively exploited.

(3) Epidemiological studies support the hypothesis that the major antioxidant nutrients vitamin $\mathrm{E}$ and vitamin $\mathrm{C}$, and $\beta$-carotene (which may or may not be acting as antioxidant in vivo), may play a beneficial role in prevention of several chronic disorders. More research is needed on the impact of other non-nutrient compounds, such as other carotenoids and flavonoids, on human health. In general, human intervention studies using hard end-points are the gold standard. Trials are restricted mainly to the major antioxidants and do not allow firm conclusions because of inconsistent findings, an insufficient number of studies and the use of varying doses. There is evidence that large doses of $\beta$-carotene may be deleterious to the health of certain subgroups of the population such as heavy habitual smokers.

(4) With respect to the safety of administration of supplementary vitamins, vitamin C is safe at levels of supplementation up to $600 \mathrm{mg} / \mathrm{d}$, and higher levels, up to $2000 \mathrm{mg} / \mathrm{d}$, are without risk. Vitamin $E$ has a very low human toxicity and an intake of $1000 \mathrm{mg} / \mathrm{d}$ is without risk; $3200 \mathrm{mg} / \mathrm{d}$ has been shown to be without any consistent risk. Large intakes of $\beta$-carotene must be viewed with caution because they have been shown to confer detriment to a population at high risk of lung cancer when administered after many years of high risk (smoking) behaviour. Until further work clarifies the situation in heavy smokers with respect to taking supplements, larger doses should be avoided by such individuals. There is little reliable information about the human toxicology of flavonoids and related non-nutrient antioxidant constituents of the diet. 
(5) The food industry has long experience in the control of oxidative damage in foods and this experience can be used to advantage for the protection of food antioxidants which are beneficial. Some of these, such as vitamins $\mathrm{C}$ and $\mathrm{E}$ and $\beta$-carotene, are well known, and strategies for their protection in foods are already exploited by food technologies. Food technology strategies for the preservation of those antioxidants which have been shown to be beneficial to health can be applied in a cost-effective manner.

\section{Research needs}

(1) The review of the available scientific database enables the identification of areas where further research is required. Improvement in dietary antioxidant intake in human populations is expected to result in lowering of the risk of a number of degenerative diseases. While desirable as an ultimate objective per se, the impact on public health and the resultant decrease in health-care costs make it imperative that substantial sums of money should be spent on research in this important area.

(2) Direct measurement of prooxidants in vivo is difficult or impossible. It is imperative to establish which are the critical free radical 'hits' that are the relevant ones in the aetiology of diseases. Are the processes examined really relevant to the disease causation? There is a need to identify which are the important antioxidants in terms of the maintenance of health, and what is their relationship to one another. Clarification is needed as to whether it is the antioxidant role of the substance that is important, or whether it is some other function, and the possible nonantioxidant effects of antioxidants, in particular with respect to modulation of gene expression, also need further research. The primary aim is to identify the active components in the overall system that promote health.

(3) Ex vivo methodologies for quantitating and validating damage in vivo to biological macromolecules urgently need further attention before meaningful work can proceed on providing evidence of the level of antioxidants needed to maintain health and well-being. It is necessary to refine and validate methods that are already available for measurement of oxidative damage in human subjects in a non-invasive manner. Validation in many centres by measurement of oxidative damage in the same biological material needs to be undertaken using the same methodology. There is a somewhat longer term need for development of techniques to be used $e x$ vivo as measures of protein oxidation in vivo.

(4) Nutritional options modulating oxidative damage. For proper epidemiological research, as well as for human intervention studies, it would be desirable to put special emphasis on the following. (a) Chemical analysis of the antioxidant content of foods. (b) Studies of bioavailability of antioxidants from the diet, and the factors that influence the absorption, distribution and tissue uptake of the compounds and the likely impact of the antioxidants on metabolic processes. (c) Development and validation of biomarkers of intermediate end-points, both biological response markers and early disease markers, and emphasis on the relevance of the biomarker to the disease end-point as well as the disease process. (d) Application of the validated biomarkers of intermediate end-points in randomized controlled trials testing the efficacy of antioxidants in functional foods for the maintenance of health and well-being.

(5) Safety implications of nutritional enhancement of antioxidants. The detailed evidence that is already available which demonstrates that vitamin $C$ and vitamin $E$ are safe at quite high levels of inclusion in the human diet, means that is unnecessary to recommend further work in this area. The safety of $\beta$-carotene was not questioned before the results of the Finnish and American intervention studies, which showed an apparent exacerbation in the incidence of lung cancer in heavy smokers who were given supplements of $\beta$-carotene. This observation needs urgent clarification. With respect to the flavonoids and other polyphenols, it is likely that their bioactivity will be explored, and the key question of their bioavailability clarified, in the near future. It will be necessary to examine the safety of such bioflavonoid compounds.

(6) With regard to the role of food technology, there is no particular direction that research needs to take at the present time. Developments in food technology will be based on, and adapt to, nutritional recommendations resulting from biologically driven work.

\section{Prooxidants: Antioxidants: Oxidative damage: Diet and health}

\section{'Good health is more than the mere absence of disease'. Mark Twain}

\section{Introduction}

This paper presents a comprehensive assessment of the literature to the end of December 1997 relevant to the role and importance of dietary antioxidants in human health.
Section 2 reviews the basic science concerning free-radical damage and the ameliorating role at the cellular level of antioxidants, and introduces the concept of maintenance of health, and the prevention of some major human degenerative diseases. In order to assess the extent of free-radical damage in human subjects in vivo, and the modifying effects of antioxidants, it is necessary to have valid, precise biomarkers. Present literature on this topic is reviewed in 
section 3. An account is given in section 4 of the human epidemiological and interventional evidence that links a high intake of dietary antioxidants with a low risk of degenerative disease. If it should be proven with reasonable certainty that antioxidants do indeed lower the risk of human degenerative disease, it is essential to be sure that intervention with antioxidants in the diet, by fortification or supplementation of foods, is entirely free from harmful sideeffects; this important topic is addressed in section 5, and the possible contribution of the food industry and food technologists to this enhancement is discussed in section 6. The 7th and 8th sections assess the 'state of the art' with respect to the foregoing sections and make recommendations for research in the immediate future.

\section{Oxidative damage, antioxidant defence and the role of prooxidants in disease}

\subsection{Antioxidant defence system of the human organism}

2.1.1. Origins and nature of free-radicals and other oxidants. The development and existence of an organism in the presence of $\mathrm{O}_{2}$ is associated with the generation of reactive oxygen species (ROS), even under physiological conditions. ROS are responsible for the oxidative damage of biological macromolecules such as DNA, carbohydrates and proteins (Halliwell \& Gutteridge, 1989; Sies, 1991; Halliwell, 1996). These processes are discussed as pathobiochemical mechanisms involved in the initiation or progression phase of various diseases (Diplock, 1994; Wiseman \& Halliwell, 1996). Some of the most relevant ROS are: peroxyl radicals (ROO'), the nitric oxide radical $\left(\mathrm{NO}^{\circ}\right)$, the superoxide anion radical $\left(\mathrm{O}_{2}^{-}\right)$, singlet oxygen $\left({ }^{1} \mathrm{O}_{2}\right)$, peroxynitrite $\left(\mathrm{ONOO}^{-}\right)$, and hydrogen peroxide $\left(\mathrm{H}_{2} \mathrm{O}_{2}\right)$. ROS are either radicals (molecules that contain at least one unpaired electron) or reactive nonradical compounds, capable of oxidizing biomolecules. Therefore, these intermediates are also called oxidants or prooxidants (Halliwell \& Gutteridge, 1989; Sies, 1991).

There are various sources for specific ROS in the human organism. However, the superoxide radical anion appears to play a central role, since other reactive intermediates are formed in reaction sequences starting with $\mathrm{O}_{2}^{--}$. It is generated by enzymic one-electron reduction of $\mathrm{O}$ from xanthine oxidase ( $E C$ 1.2.3.2), NADPH oxidase, or by leakage of the respiratory chain. It has been estimated that about $1-3 \%$ of the $\mathrm{O}_{2}$ we utilize is converted to $\mathrm{O}_{2}^{--}$ (Fridovich, 1986; Halliwell, 1996).

$\mathrm{H}_{2} \mathrm{O}_{2}$ is a non-radical reactive species and can easily diffuse between living cells. It is efficiently converted to water by the enzyme catalase $(E C 1.11 .1 .6)$, a process which determines its half-life. Recent evidence suggests that $\mathrm{H}_{2} \mathrm{O}_{2}$ is involved in signal transduction regulating the expression of genes through the nuclear factor $\kappa \mathrm{B}$ and apoprotein-1 pathways (Schreck \& Baeuerle, 1994; Sen \& Packer, 1996).

The most reactive species is the hydroxyl radical with an estimated half-life of about $10^{-9} \mathrm{~s}$. It might be formed in vivo on high-energy irradiation (e.g. X-rays) by homolytic cleavage of body water or from endogenous $\mathrm{H}_{2} \mathrm{O}_{2}$ in metal-catalysed processes (Fenton reaction: Fe-catalysed Haber-Weiss reaction). u.v.-Light is insufficiently energetic to split water but it can cleave $\mathrm{H}_{2} \mathrm{O}_{2}$ to yield two molecules of the hydroxyl radical. The high reactivity of this radical implies immediate reaction at the place where it is generated.

The peroxyl radical (ROO') is relatively long lived (seconds) with a considerable diffusion pathlength in biological systems. It can be generated in the process of lipid peroxidation which is initiated by the abstraction of an $\mathrm{H}$ atom from polyunsaturated fatty acids (PUFA); the hydro$\mathrm{xyl}$ radical is capable of starting this reaction sequence (Esterbauer et al. 1992; Reaven \& Witzum, 1996).

Further products generated in lipid peroxidation are alkoxyl radicals $\left(\mathrm{RO}^{\circ}\right)$ and organic hydroperoxides $(\mathrm{ROOH})$. The latter might rearrange to endoperoxide intermediates which are cleaved to yield aldehydes. The reaction of aldehydes with amine groups of proteins has been discussed as a mechanism involved in the modification of the protein part of lipoproteins.

Singlet molecular oxygen $\left({ }^{1} \mathrm{O}_{2}\right)$ is another non-radical ROS which is suggested to be formed in vivo in lightexposed tissue. Its half-life has been estimated to be $10^{-6} \mathrm{~s}$ depending on the nature of the surrounding matrix. ${ }^{1} \mathrm{O}_{2}$ can interact with other molecules either by transferring its excitation energy or by combining chemically. Preferential targets for chemical reactions are double bonds; e.g. in PUFA or guanine in DNA bases (Kanofsky, 1989; Stahl \& Sies, 1993; Cadet et al. 1994).

An interesting ROS which has attracted attention within the past few years is the nitric oxide radical (NO'). It is a signalling compound formed enzymically from arginine and relaxes smooth muscles in blood-vessel walls resulting in lowered blood pressure. It is also produced by activated macrophages contributing to the primary immune defence. An excess of $\mathrm{NO}^{-}$is cytotoxic. It might react directly with biomolecules or combine with $\mathrm{O}_{2}^{--}$to form peroxynitrite $\left(\mathrm{ONOO}^{-}\right)$. Peroxynitrite is capable of inducing lipid peroxidation in lipoproteins but might also interfere with cellular signalling by nitrating tyrosine residues in proteins (Beckman, 1996; Packer, 1996).

The ROS described here, and also the biological pathways for their endogenous formation, are examples of a whole class of reactive intermediates and their ways of generation. It should further be noted that the organism is also exposed to ROS from external sources. With the diet many compounds of prooxidant nature, such as quinones capable of redox cycling, are delivered to the organism. Also an array of radicals are inhaled with cigarette smoke; ozone, of which increasing levels are reported due to air pollution, is an ROS which can oxidize lipids (Pryor et al. 1995).

ROS are also produced in the organism as a part of the primary immune defence. Phagocytic cells such as neutrophils, monocytes, or macrophages defend against foreign organisms by synthesizing large amounts of $\mathrm{O}_{2}^{--}$or $\mathrm{NO}$ as a part of their killing mechanism. Several diseases are accompanied by excessive phagocyte activation resulting in tissue damage which is at least in part due to the activity of ROS.

2.1.2. Enzymic and non-enzymic defence systems in vivo. To counteract the prooxidant load a diversity of antioxidant defence systems are operative in biological systems including enzymic and non-enzymic antioxidants. An antioxidant has been defined as 'any substance that, when present in low concentrations compared to that of an 
oxidizable substrate, significantly delays or inhibits the oxidation of that substrate' (Halliwell \& Gutteridge, 1989; Sies, 1993; Halliwell, 1995).

The major enzymes directly involved in the detoxification of ROS are superoxide dismutase ( $E C$ 1.15.1.1), scavenging $\mathrm{O}_{2}^{-}$, as well as catalase and glutathione peroxidases $(E C$ 1.11.1.9) which reduce $\mathrm{H}_{2} \mathrm{O}_{2}$ and organic hydroperoxides respectively. Several subtypes of glutathione peroxidase are Se-dependent. In animal studies an elevated intake of Se was associated with protective effects against cancer. Its preventive effects in man are still under investigation (Levander \& Burk, 1996). Indirect antioxidant functions are mediated by enzymes that restore endogenous antioxidant levels; e.g. GSH levels are replenished on reduction of GSSG by glutathione reductase ( $E C$ 1.6.4.1). Further, reactive intermediates produced in reactions of prooxidants and biological molecules (e.g. epoxides) are conjugated by phase II detoxification enzymes such as glutathione- $S$ transferases (EC 2.5.1.18) to favour their excretion. Another strategy to prevent the formation of ROS is the control of the levels of free $\mathrm{Fe}$ or $\mathrm{Cu}$ ions. Metal-binding proteins responsible for the transport of these ions bind them tightly, thus preventing the initiation of lipid peroxidation or DNA damage. Some of the most relevant metal-binding proteins are ferritin, transferrin, and caeruloplasmin.

Various endogenous low-molecular-mass compounds are also involved in antioxidant defence. GSH, the major cytosolic thiol, serves as a cofactor for several detoxifying enzymes (glutathione peroxidases, glutathione- $S$-transferases), is involved in the reduction of protein disulfides and additionally scavenges ROS, being oxidized to GSSG. Other endogenous compounds such as ubiquinol-10, urate, or bilirubin also exhibit antioxidant activities (Jacob \& Burri, 1996).

2.1.3. Dietary antioxidants; nutrient and nonnutrient. The human diet contains an array of different compounds that possess antioxidant activities or have been suggested to scavenge ROS based on their structural properties. The most prominent representatives of dietary antioxidants are ascorbate (vitamin C), tocopherols (vitamin E), carotenoids, and flavonoids. Apart from vitamin C, each group of these antioxidants consists of a number of structurally different compounds; e.g. more than 600 different carotenoids have been identified to date and about fifty of them might occur in the human diet (Sies \& Stahl, 1995; Rice-Evans \& Miller, 1996; Rock et al. 1996). In the diet, there may be synergistic effects of these various dietary compounds which are difficult to assess at present. Indeed, the diet may be considered as an orchestra where interactions between constituents may bring about effects which are not the necessary properties of the individual constituents.

Vitamin $C$ is considered to be one of the most powerful, least toxic natural antioxidants (Bendich et al. 1986; Weber et al. 1996). It is water-soluble and is found in high concentrations in many tissues; human plasma contains about $60 \mu \mathrm{mol}$ ascorbate/l. On interaction with ROS it is oxidized to dehydro-ascorbate via the intermediate ascorbyl free radical. Dehydro-ascorbate is recycled back to ascorbic acid by the enzyme dehydro-ascorbate reductase. Thus, dehydro-ascorbate is found in only very low levels compared with ascorbate. As a scavenger of ROS ascorbate has been shown to be effective against the superoxide radical anion, $\mathrm{H}_{2} \mathrm{O}_{2}$, the hydroxyl radical, and singlet oxygen. In aqueous solutions vitamin $\mathrm{C}$ also scavenges reactive nitrogen oxide species efficiently, preventing the nitrosation of target molecules. The major sources of ascorbate in the diet are fruits, especially citrus fruits, kiwi fruit, cherries and melons, and vegetables such as tomatoes, leafy greens, broccoli, cauliflower, Brussels sprouts, and cabbage; its content might exceed $100 \mathrm{mg}$ ascorbate $/ 100 \mathrm{~g}$ fresh weight. At low dose levels $(100 \mathrm{mg})$ the bioavailability values for vitamin $\mathrm{C}$ from synthetic and food sources are very similar (Mangels et al. 1993a); the efficacy of absorption decreases with increasing dose levels (Levine et al. 1996). There is evidence from studies in vitro that vitamin $\mathrm{C}$ is capable of regenerating tocopherol from the tocopheroxyl radical which is formed on inhibition of lipid peroxidation by vitamin $\mathrm{E}$ (Niki et al. 1982, 1985). This process would allow for the transport of a radical load from a lipophilic compartment to an aqueous compartment where it is taken care of by efficient enzymic defence systems. It should be noted, however, that ascorbate might also act as a prooxidant in vivo. In the presence of free transition metal ions ( $\mathrm{Fe}$ and $\mathrm{Cu}$ ) and ascorbate the hydroxyl radical can be generated and initiation of lipid peroxidation may occur. However, the amounts of free transition metals in vivo are very small because they are efficiently bound to proteins. Vitamin $\mathrm{C}$ has additional well-established biological functions including cofactor-activity for several important enzymes (Levine et al. 1996).

The term vitamin $\mathrm{E}$ is a generic description for all tocols and tocotrienol derivatives which exhibit the biological activity of $\alpha$-tocopherol (Parker, 1989; Eldin \& Appelqvist, 1996; Sokol, 1996; Traber \& Sies, 1996). This group of compounds is highly lipophilic, and operative in membranes or lipoproteins. Their most important antioxidant function appears to be the inhibition of lipid peroxidation, scavenging lipid peroxyl radicals to yield lipid hydroperoxides and a tocopheroxyl radical. The latter is less reactive than peroxyl radicals towards neighbouring PUFA and acts as a chain-breaking antioxidant. The tocopheroxyl radical might be either reduced by ascorbate and GSH or further oxidized to the respective quinone. Since only small amounts of tocopheryl quinone are detectable in human blood and tissues, the regenerative pathway in vivo appears to be favoured. In comparison with other lipophilic antioxidants, $\alpha$-tocopherol is probably the most efficient in the lipid phase (Niki, 1987). It contains shielding methyl groups adjacent to the phenolic hydroxyl group and it is optimally positioned in membranes by its phytyl side-chain, which is located in the hydrophobic region of the membrane structure. In addition to its peroxyl-radical scavenging properties, further interactions with ROS have been described, including quenching of singlet oxygen and interaction with peroxynitrite. The richest sources of vitamin $\mathrm{E}$ in the diet are vegetable oils (soyabean, maize, cottonseed, and safflowerseed), and products made from these oils such as margarine and mayonnaise. Further, wheat germ, nuts, and some green leafy vegetables contribute considerable amounts to the vitamin E supply (Parker, 1989). Vitamin E plasma levels in man are about $22 \mu \mathrm{mol} / 1$; the compound is also found in tissues such 
as liver, kidney, fat and adrenals. In the liver the RRRisomer of $\alpha$-tocopherol is preferentially incorporated into VLDL which are further catabolized in the circulation. Thus, RRR- $\alpha$-tocopherol is the major form of vitamin E in LDL (Traber \& Sies, 1996).

Carotenoids are natural colourants with pronounced antioxidant activity (Stahl \& Sies, 1993; Olson \& Krinsky, 1995). Their chemical properties are closely related to the presence of an extended system of conjugated double bonds which is substituted with various endgroups. ROS which are efficiently scavenged by carotenoids are ${ }^{1} \mathrm{O}_{2}$ and peroxyl radicals (Palozza \& Krinsky, 1992). Two different pathways are operative with respect to the deactivation of ${ }^{1} \mathrm{O}_{2}$ : physical and chemical quenching (Truscott, 1990). Physical quenching implies the deactivation of ${ }^{1} \mathrm{O}_{2}$ by energy transfer from the excited oxygen species to the carotenoid, yielding a triplet excited carotenoid. The energy of the excited carotenoid is dissipated through vibrational interactions with the solvent to recover ground state carotenoid. The carotenoid remains intact in this process and might undergo further cycles of deactivation. Chemical quenching contributes less than $0.05 \%$ to total ${ }^{1} \mathrm{O}_{2}$-quenching by carotenoids but is responsible for the eventual destruction of the molecule. Carotenoids are the most efficient naturally occurring quenchers for ${ }^{1} \mathrm{O}_{2}$ with quenching rate constants of about $5-12 \times 10^{9} / \mathrm{mol}$ per s. Carotenoids were reported to scavenge peroxyl radicals by chemical interaction (Kennedy \& Liebler, 1992). It is suggested that carotene radical intermediates are formed in this process which finally leads to the destruction of the molecule. Like vitamin E, carotenoids belong to the group of lipophilic antioxidants present in lipoproteins such as LDL and HDL. It has been shown that they are consumed when isolated LDL is exposed to the process of lipid peroxidation. Their contribution to the antioxidant defence system of LDL is not clear, since no regeneration pathways for oxidized carotenoids are known at present. A variety of structurally different carotenoids are present in fruits and vegetables. Some of the major sources are carrots ( $\alpha$-carotene, $\beta$-carotene), tomatoes (lycopene), citrus fruits ( $\beta$-cryptoxanthin), spinach (lutein), and maize (zeaxanthin) (Mangels et al. 1993b). The absorption and transport processes of carotenoids are quite complex. Several factors influencing carotenoid bioavailability from food, such as co-ingestion of fat or fibre, cooking or food processing, have been identified (Erdman et al. 1993).

Flavonoids are a large group of polyphenolic antioxidants that occur in several fruits, vegetables, and beverages such as tea, wine and beer mainly as $O$-glycosides. They are efficient antioxidants capable of scavenging radical species (peroxyl radicals, hydroxyl radical, $\mathrm{O}_{2}^{-}$) forming a phenoxy radical (Rice-Evans et al. 1995; Rice-Evans \& Miller, 1996). The term flavonoids summarizes a number of structurally different subgroups including flavanols (catechin, epicatechin), flavonols (quercetin, myricetin, kaempherol), flavanones (naringenin, taxifolin), flavones (apigenin, hesperetin), isoflavones (genestein), or anthocyanidins (cyanidin, malvidin). Several criteria for optimal radical scavenging properties of flavonoids have been postulated based on pulse radiolysis studies. These include the presence of the $3^{\prime}, 4^{\prime}$-dihydroxy structure in ring $B$, the presence of the 2,3-double bond in conjugation with the 4oxo-group in ring $\mathrm{C}$, and the presence of a 5-hydroxyl group in ring A with a 3-hydroxyl group and a 4-oxo function in the C-ring. The antioxidant properties of flavonoids have been investigated in various studies in vivo and in vitro. It should be mentioned, however, that the bioavailability of these compounds is rather poor. They are rapidly conjugated in phase II detoxification reactions and levels of free flavonoids in human plasma are very low. Further phenolic compounds with antioxidant activity are derivatives of cinnamic acid; e.g. caffeic acid, chlorogenic acid, and ferulic acid (Rice-Evans \& Miller, 1996).

In addition to the flavonoids, a number of other phenolic compounds of potential interest occur in foods. Thus, olive oil contains a number of phenolic substances, notably the $o$ diphenol tyrosol, which may contribute to the antioxidant content of diets rich in olive oil (Kiritsakis, 1990). Similarly, plants of the Lamiaceae family, notably rosemary, oregano, sage, mint and thyme, contain a range of potential antioxidants such as carnosol, rosemanol and carvacrol, which can contribute to the antioxidant potential of the diet (Lagouri \& Boskou, 1996). As with the flavonoids, however, little is known of the human absorption and tissue distribution of these compounds.

Several other dietary constituents might also be involved in the antioxidant defence system either by direct action as antioxidants or by effects related to the induction of detoxifying enzymes. Enzymes such as glutathione peroxidase and superoxide dismutase, which require a dietary supply of $\mathrm{Se}$, and of $\mathrm{Cu}$ and $\mathrm{Zn}$ respectively, contribute to the overall oxidative defence mechanism. Some endogenous substances such as urate also add to the antioxidant potential of living cells, although their significance is only speculative. Enhancement of dietary intake of the minerals identified may be beneficial when their content in the diet is low.

\subsection{Oxidative damage to bodily functions and its implications in disease}

ROS are suggested, or known, to be involved in pathogenic processes of numerous diseases (Esterbauer et al. 1992; Luis \& Navab, 1993; Diplock, 1994; Sies, 1997) such as cardiovascular disease, some forms of cancer, cataract, age-related macular degeneration, rheumatoid arthritis and a number of neurodegenerative diseases. Oxidative damage to important biomolecules is a deleterious pathway, but also influences of ROS on gene regulation or the immune system might impair bodily functions. There is increasing evidence from clinical and intervention studies, as well as from basic research that antioxidants might prevent or delay the development of disease states. There may also be particular population groups that will benefit from enhanced antioxidant intake, such as pregnant women, neonates and children, senior citizens and, perhaps, sportspeople.

2.2.1. Coronary heart disease. The primary cause for most cardiovascular diseases is thought to be arteriosclerosis, a multifactorial disease of the artery wall. It is suggested that in the early stages of arteriosclerosis lipid deposits, so-called fatty streaks, are formed in the subendothelial space. There is increasing evidence that 
oxidative stress, particularly oxidation of LDL, is a risk factor and plays a role in the pathogenic pathway (Berliner \& Heinecke, 1996). LDL oxidation is due to a lipid peroxidation reaction initiated by free radicals. Separate investigations of the lipid and protein parts of oxidized LDL demonstrated that oxidative modifications of both contribute to the proatherogenic properties of oxidized LDL. Several biochemical mechanisms underlying this effect have been discussed. These include the formation of foam cells on the uptake of oxidized LDL via the scavenger receptor by macrophages resident in the subendothelial area, release of cytotoxic lipid peroxidation products from oxidized LDL, or chemoattractant properties of the oxidized lipoprotein. LDL oxidation is efficiently inhibited by lipophilic antioxidants of which $\alpha$-tocopherol appears to be the most important. Epidemiological studies suggest preventive effects towards atherogenic lesions to be associated with an increased uptake of lipophilic antioxidants such as vitamin $\mathrm{E}$ or carotenoids (Rimm et al. 1993).

Additional effects of RRR- $\alpha$-tocopherol, independent of its antioxidant activity, have been related to the protective properties of this compound. An early event in the onset of arteriosclerosis is the migration of smooth-muscle cells from the media to the intima of the arterial wall followed by proliferation of these cells. There is increasing evidence that RRR- $\alpha$-tocopherol acts as a negative regulator of smooth-muscle cell proliferation via modulation of protein kinase $\mathrm{C}$ activity. Protein kinase $\mathrm{C}$ is an important element in the signal transduction cascade mediated by growth factors such as platelet-derived growth factor which are involved in the control of cell proliferation. It should be noted that these effects are limited to RRR- $\alpha$-tocopherol; RRR- $\beta$-tocopherol does not inhibit protein kinase C (Azzi et al. 1995; Özer et al. 1995).

2.2.2. Carcinogenesis. Carcinogenesis is a complex multistep process including initiation, promotion and progression. The generation of ROS is thought to be linked to tumourigenesis at different levels. Oxidative damage to DNA has been demonstrated in vitro and in vivo leading to DNA single or double strand breaks and DNA cross linking, as well as to chromosomal aberrations such as breakage or rearrangement. Modified DNA bases (e.g. hydroxythymidine, or hydroxyguanine) have been determined after exposure of cells to situations of oxidative stress. The modification of DNA bases might result in point mutations, deletions, or gene amplification as a first step of carcinogenesis. Further, ROS are capable of deactivating detoxifying enzymes responsible for the scavenging of potent carcinogens. Data from epidemiological studies support the idea that antioxidants are preventive in carcinogenesis by scavenging ROS (Flagg et al. 1995).

Carotenoids exhibit further biological functions which are not related to their antioxidant activities but might be of importance with respect to their cancer preventive effects (Gerster, 1995). It has been shown that provitamin A and non-provitamin A carotenoids are capable of inhibiting the growth of transformed fibroblasts (Bertram \& Bortkiewicz, 1995). There is increasing evidence that growth arrest is due to the stimulation of gap-junctional communication between transformed and surrounding normal cells. These findings suggest that carotenoids or carotenoid-derived retinoids play a role in intercellular signalling involved in growth control. Inhibitory effects of $\beta$-carotene and lycopene on cell proliferation have also been described for several human cancer cell lines (Sharoni \& Levy, 1996).

2.2.3. Cataract and age-related macular degeneration. Oxidative damage and impaired vision have been discussed in the context of two ophthalmological diseases of the elderly, cataract and age-related macular degeneration (AMD) (Schalch, 1992; Taylor, 1993). Senile cataract indicates the opacity of ocular lenses. Lens proteins are extremely longlived and often show oxidative damage. This is not surprising, since they are subjected to chronic exposure to light and $\mathrm{O}_{2}$, which is likely to be responsible for the formation of ROS which might react with lens proteins. As a consequence, the damaged proteins may aggregate and precipitate, thus losing their regular function. Supplementation studies support the hypothesis that a higher intake of vitamins including vitamin $\mathrm{C}$ and vitamin $\mathrm{E}$ prevents or delays the development of cataracts (Seddon et al. 1994b).

AMD is the major cause of visual impairment in Western countries and affects the anatomical region of the retina with the highest degree of visual activity. The macular pigment (yellow spot) represents a colour filter through which light must pass before detection. The carotenoids lutein and zeaxanthin are the predominant pigments in this area (Landrum, 1997). Carotenes (hydrocarbon carotenoids) are not present in the yellow spot. The function of the macular pigment has not been unequivocally identified but it might protect against photo-oxidation by blue light, mediated by excited triplet state molecules, ${ }^{1} \mathrm{O}_{2}$, or superoxide. There are hints from food-frequency questionnaires that an increased consumption of food rich in lutein and zeaxanthin is associated with a diminished risk of AMD (Seddon et al. 1994a). Carotenoids are the most efficient natural compounds scavenging ${ }^{1} \mathrm{O}_{2}$ and excited triplet state molecules.

2.2.4. Neuronal diseases. Growing data from experimental models and human brain studies add evidence that oxidative stress might play a role in the development of neuronal degeneration related to diseases such as Parkinson's disease, amyotrophic lateral sclerosis, and Alzheimer's disease (Kondo, 1996; Simonian \& Coyle, 1996). ROS are capable of inducing both necrosis and apoptosis. As a consequence of lipid peroxidation membrane rupture might occur or ion gradients, operative over compartments which are separated by membranes, might be disturbed. Neurons might undergo necrotic cell death as has been demonstrated in cell culture following depletion of intracellular GSH, the major endogenous antioxidant thiol. NO has been hypothesized to be an important mediator of neuronal death under pathological conditions. The ultimate species responsible for NO toxicity may be peroxynitrite which is formed by the reaction of the NO-radical with the superoxide radical.

Beyond the classical aspects of oxidative damage to biologically relevant molecules as pathological mechanisms underlying several diseases, and the protective effects of antioxidants, new fields of research in this area are rapidly developing. This includes effects of prooxidants and antioxidants on immune functions (Bendich, 1990) and antioxidant and redox regulatory properties on gene expression. Both mechanisms may be involved in the development of 
disease states while protection might be provided by antioxidants via these pathways.

\subsection{Conclusions}

An imbalance between ROS and antioxidant defence systems may lead to chemical modifications of biologically relevant macromolecules like DNA, proteins or lipids which are possible pathobiochemical mechanisms in the initiation or development of several disease states. Experimental data provide evidence that dietary antioxidants scavenge ROS and are useful in the prevention of these diseases. Epidemiological studies clearly show a correlation between the increased consumption of food rich in antioxidants and a decreased risk of several diseases. Thus, an increased intake of fruits and vegetables can be recommended. Data on antioxidant supplementation are contradictory. Further research is necessary to establish whether supplementation beyond dietary intake levels is of benefit.

\section{Available methodologies for evaluating and quantifying ex vivo damage to DNA, lipids and proteins by prooxidants in vivo}

\subsection{Oxidative damage to DNA}

The most abundant base alteration induced in DNA by ROS is the formation of 8-oxo-7,8-dihydro-2'-deoxyguanine (8oxodG). In vivo this DNA base alteration is repaired by excision and the resulting product 8-oxodG is excreted unchanged, and independently of diet, into the urine. Thus, the rate of excretion of 8-oxodG (as given by the appearance of the metabolite in urine with time) serves as a biological marker of the integrated rate of oxidative DNA damage in the whole body.

DNA damage is usually measured in lymphocytes isolated from blood or in urine. Baseline levels of DNA damage are considered to be important because repair may be incomplete (one damaged base per $10^{6}$ bases); the actual measurement may therefore provide an estimate of the balance between damage and repair, so the time window is a crucial consideration here. From studies in vitro it is known that when DNA is exposed to the activated oxygen species the product is specific to the oxygen species involved, thus:

$$
\begin{gathered}
{ }^{1} \mathrm{O}_{2}, \mathrm{ROO}^{-} \rightarrow \text { guanine oxidized } \\
\mathrm{OH} \rightarrow \text { multiplicity of changes to all four bases } \\
\mathrm{O}_{2}^{--}, \mathrm{H}_{2} \mathrm{O}_{2} \rightarrow \text { no base changes } \\
\mathrm{ONOO}^{-} \rightarrow \text { xanthine, hypoxanthine, 8-nitroguanine. }
\end{gathered}
$$

There are two types of measurement of oxidative DNA damage. First, steady-state damage can be measured when DNA is isolated from human cells and tissues and analysed for base damage products: it presumably reflects the balance between damage and DNA repair. Hence a rise in steadystate oxidative DNA damage (e.g. as has been reported in some human cancerous tissues; Malins \& Haimonot, 1991; Olinski et al. 1992) could be due to increased damage and/or decreased repair. Second, several DNA base damage products are excreted in human urine, including the nucleoside 8-hydroxy-deoxyguanosine (8-OHdG), 8-hydroxy-adenine and 7-methyl-8-hydroxyguanine (Ames, 1989; Stillwell et al. 1989) but the one most exploited is $8-\mathrm{OHdG}$, usually measured by a method involving HPLC with electrochemical detection (Ames, 1989; Shigenaga et al. 1994).

The validity of these urinary measurements of oxidative DNA damage must be considered. The level of 8-OHdG in urine is presumably unaffected by the diet since nucleosides are not absorbed from the gut. The question of whether any 8-OHdG is metabolized to other products in man has not been rigorously addressed. Additionally, it is possible that some or all of the 8-OHdG excreted in urine may arise not from DNA, but from deoxyGTP in the DNA precursor pool of nucleotides. An enzyme has been described which hydrolyses deoxyGTP containing oxidized guanine to prevent its incorporation into DNA (Mo et al. 1992; Sakumi et al. 1993). These uncertainties require clarification.

3.1.1. Measurement of guanine damage products in DNA by HPLC and gas chromatography-mass spectrometry $(G C-M S)$. As mentioned earlier, 8-hydroxyguanine (8$\mathrm{OHG}$ ) and $8-\mathrm{OHdG}$ are the products most frequently used as indicators of oxidative DNA damage. Analysis of $8-\mathrm{OHdG}$ using HPLC coupled to electrochemical detection (Floyd $e t$ al. 1986), is a highly sensitive technique that is frequently used after release of 8-OHdG from DNA, usually by enzymic hydrolysis. GC-MS with selective ion monitoring has also been used to characterize oxidative DNA base damage by the identification of a spectrum of products (Dizdaroglu, 1993a), including 8-OHG, after formic acid hydrolysis of DNA and derivatization (often by trimethylsilylation) to generate volatile products. When GC-MS is used to measure modified DNA bases, a quantitative analysis of these bases in a DNA sample can be achieved by adding a suitable internal standard to that sample at an early stage of the analysis, such as before the hydrolysis of the DNA (Dizdaroglu, 1993b). Stable-isotope-labelled analogues of the modified bases can also be used as internal standards (Dizdaroglu, 1993b).

One advantage of the GC-MS approach is that measurement of a wide range of base damage products allows more accurate quantification of DNA damage and can help to identify the ROS and reactive nitrogen species (RNS) that caused the damage (Malins \& Haimonot, 1991); $\mathrm{O}_{2}$ selectively attacks guanine whereas $\mathrm{OH}^{-}$attacks all four DNA bases. However, the levels of 8-OHdG measured in DNA by HPLC with electrochemical detection are often (Halliwell \& Dizdaroglu, 1992) (but not always; Lunec et al. 1994; Herbert et al. 1996) less than the levels of 8-OHG measured by GC-MS with selective ion monitoring. HPLC could underestimate the real amount of 8OHdG in DNA if the enzymic hydrolysis was incomplete; the action of the exonucleases and endonucleases used to hydrolyse the DNA may be affected by the modification of the bases (Halliwell \& Dizdaroglu, 1992; Turk \& Weitzman, 1995) and the acid $\mathrm{pH}$ often used for nuclease digestions might induce hydrolysis of 8-OHdG to 8-OHG, resulting in the loss of HPLC-detectable material. In contrast, GC-MS might overestimate 8-OHG (and perhaps other base damage products) as a result of their artifactual formation during the heating step involved in classical silylation-based derivatization procedures (Halliwell \& 
Dizdaroglu, 1992; Ravanat et al. 1995). A 'cold' derivatization procedure has been developed that should avoid this problem (Hamberg \& Zhang, 1995). The important factor is that any necessary heating stages should be done anoxically: heating DNA bases in the presence of $\mathrm{O}_{2}$ inevitably results in oxidation. Hence some of the claimed artifacts (Hamberg \& Zhang, 1995; Ravanat et al. 1995), are possibly due to failure to remove $\mathrm{O}_{2}$. However, it is difficult to remove $\mathrm{O}_{2}$ completely. Indeed, a major problem to be considered in all these techniques is the possibility that DNA is oxidatively damaged during its isolation from cells and tissues, particularly if phenol-based methods are used, since oxidizing phenols generate ROS (Claycamp, 1992; Finnegan et al. 1996). However, rigorous control of isolation procedures and avoidance of phenol in many laboratories (e.g. by studying isolated chromatin or by using different DNA isolation methods) does not abolish oxidative damage detected in isolated DNA (Halliwell \& Dizdaroglu, 1992; Dizdaroglu, 1993a; Harris et al. 1994; Shigenaga et al. 1994; Finnegan et al. 1996), strongly supporting the view that there is a low steady-state DNA damage in vivo. Indeed the presence of a DNA repair enzyme system and the excretion of base damage products support the view that oxidative damage really does occur in vivo.

As an alternative means of avoiding possible problems with derivatization an HPLC method with electrochemical detection has been developed that allows measurement of 8OHG and three of the other oxidized base products in acidhydrolysed DNA, thus avoiding the need for derivatization. Liquid chromatography-mass spectrometry techniques are under development in several laboratories: this is another approach to avoiding derivatization problems if sufficient sensitivity can be achieved.

\subsection{Oxidative damage to lipids}

3.2.1. Lipid peroxidation. There is a range of methods available for measurement of markers of lipid peroxidation and products of peroxidation in vivo that can be measured in blood and urine as indicators of oxidative stress. There are, however, a number of problems that need to be resolved before it is possible to be confident that measurements made are valid and reproducible, and inter-laboratory studies using the same reference material are urgently needed to resolve the remaining difficulties. These include variability of the standards used, and small differences in the technique employed which can have a marked effect on the result achieved; furthermore, no single method can, by itself, provide an unambiguous indicator of levels of lipid peroxidation, whether by measuring lipid hydroperoxides, or degradation products therefrom.

Evidence for damage to lipids in vivo is derived from measurement of peroxides or isoprostanes in blood and urine. Such indicators of peroxides in vivo are important $v i s-a ̀-v i s$, for example, the relationship of plasma peroxides to vessel wall oxidation of LDL in the context of atherosclerosis. The major problem which has yet to be addressed with some consolidated approach is the differentiation in identification of peroxides formed as a consequence of in vivo oxidative stress and those ingested from dietary sources.
Peroxide levels in cells and tissues present a balance between peroxide formation and peroxide metabolism or decomposition, i.e. they are essentially a 'steady-state' measurement.

With respect to measurement of levels of lipid hydroperoxides, a number of methods are available. Ex vivo measurement of the lipid hydroperoxide products directly is best achieved by HPLC determination following partitioning of the hydroperoxide into a polar solvent, which achieves a primary separation between less polar triacylglycerol and cholesterol hydroperoxides and the more polar free fatty acids and phospholipid hydroperoxides (RiceEvans et al. 1991). Chemiluminescence-based detection has proved very satisfactory in providing a reliable assay procedure (Yamamoto, 1994); an alternative is luminolchemiluminescence.

The steady-state levels of peroxides in human body fluids, such as blood plasma, appear very low, usually $<100 \mathrm{nmol} / \mathrm{l}$. These data come from assays that measure 'real' lipid peroxides (Holley \& Slater, 1991; Akasaka et al. 1995) viz by HPLC with chemiluminescence detection rather than notoriously-unspecific methods such as diene conjugation or the simple thiobarbituric acid (TBA) test (Halliwell \& Chirico, 1993). HPLC-based TBA tests can, however, record comparably-low values, provided that butylated hydroxytoluene is added with the TBA reagents (Halliwell \& Chirico, 1993).

Several other different approaches have been used for measurements of the lipid hydroperoxide products of peroxidation of PUFA. The most simple method conceptually involves direct iodometric determination of lipid hydroperoxide. A further alternative is provided by the ferrous oxidation in xylenol orange (FOX) method in its two variants which provide methods for measuring low levels of soluble hydroperoxides in the aqueous phase, or lipid hydroperoxides derived from membranes of lipoproteins in the lipid phase (Wolff, 1994). Assays of human tissues and body fluids by simple 'peroxide-determinations' such as those involving xylenol orange (Jiang et al. 1992) or iodometric methods (Thomas et al. 1989) could measure protein peroxides; this could conceivably explain why levels of alleged 'lipid peroxides' measured by such techniques in human body fluids tend to be higher (often in the $\mu \mathrm{M}$ range) than those revealed by the more-specific techniques for measuring lipid peroxides that were discussed earlier (see section 3.2.3).

A more recently introduced type of assay concerns measurement of isoprostanes which are derived from PUFA by a non-cyclooxygenase-mediated free-radicalcatalysed mechanism. Formation of the arachidonic acidderived compounds involves formation of four positional peroxyl radical isomers of the fatty acid which undergo endocyclization to prostaglandin ( $\mathrm{PG} \mathrm{G}_{2}$-like compounds that are then reduced to $\mathrm{PGF}_{2}$-like compounds. Four $\mathrm{F}_{2}$ isoprostane isomers are formed, each of which can, in theory, comprise eight diastereoisomers. Quantification of $F_{2}$-isoprostanes represents a reliable and useful approach to assessment of lipid peroxidation and oxidant stress in vivo (Morrow \& Roberts, 1994).

Human body fluids also contain low levels of $F_{2}$-isoprostanes, compounds isomeric to prostaglandins that appear to 
arise by free-radical oxidation of phospholipids containing arachidonic acid (Morrow \& Roberts, 1994; Morrow et al. 1995). Isoprostanes appear to exist in human plasma largely esterified to phospholipids rather than 'free', and sensitive assays to measure them have been described (Morrow \& Roberts, 1994; Wang et al. 1995). Isoprostanes and their metabolites can be measured in human urine (Morrow \& Roberts, 1994; Morrow et al. 1995) by GC-MS and this may prove a valuable assay of whole-body lipid peroxidation if a confounding effect of diet can be ruled out. These compounds are useful 'markers' of lipid peroxidation and can be measured in plasma (35 (SD 6) $\mathrm{pg} / \mathrm{ml}$ ) and urine (1600 (SD 600) pg/mg creatinine) of healthy volunteers, indicative of ongoing lipid peroxidation even in healthy human subjects (Halliwell, 1996).

3.2.2. Can some measure of 'total' peroxidation be obtained? Approaches to measurements of 'total-body' lipid peroxidation have been by measurements of urinary TBA-reactive substances (TBARS), using the HPLC TBA method (Chirico \& Halliwell, 1994), measurements of hydrocarbon gas excretion and by measurements of $\mathrm{F}_{2}$ isoprostanes in urine. Urinary TBARS measurements have been found to be confounded by a multiplicity of urinary constituents that react with TBA, and this problem is further complicated by contributions from dietary constituents, particularly cooked meats. Most of the lipid-related TBARS appearing in urine seem to arise from lipid peroxides or aldehydes in ingested food, which are presumably largely generated during cooking (Dhanakoti \& Draper, 1987; Brown et al. 1995). Hence urinary TBARS is not a suitable assay to assess whole-body lipid peroxidation in response to changes in dietary composition, although it could theoretically be used to look at effects of supplementary antioxidants in individuals on a standardized 'fixed diet' (Dhanakoti \& Draper, 1987). In any case, HPLC must be used to separate the real (TBA) ${ }_{2}$ malondialdehyde adduct since the majority of the TBARS in urine are not even lipidderived (Gutteridge \& Tickner, 1978) or derive from a wide variety of aldehydes other than malondialdehyde.

Measurement of hydrocarbon gases (alkanes and alkenes), degradation products of lipid peroxidation in vivo (Burk \& Ludden, 1989; Springfield \& Levitt, 1994), can be confounded by interference from air pollutants and from the products of gastrointestinal bacterial metabolism. They are unreliable also because of the low level of the metabolites to be measured, which challenges the sensitivity of the assay, and the possibility of metabolism of the metabolite before excretion, so that the measured amount represents only a portion of the true level; co-elution of other metabolites which are indistinguishable from the products of interest is a further problem; the method is therefore not considered further here.

There appears to be a general consensus that the most reliable assay procedures available are the chemiluminescence-linked HPLC determination of lipid hydroperoxides (Yamamoto, 1994) and the HPLC-linked TBA measurement (Yamamoto, 1994). The isoprostane assay is gaining momentum and has been excellently reviewed recently (Morrow \& Roberts, 1996).

3.2.3. LDL oxidation. Particular techniques have been developed to determine the oxidation of LDL and a wide range of methods is now available (Esterbauer et al. 1992). A critical review of practical approaches to LDL oxidation has also appeared recently (Rice-Evans et al. 1996).

The xylenol orange assay or FOX assay describes a sensitive spectrophotometric system for detecting authentic peroxides in LDL and has been successfully applied to the measurement of lipid hydroperoxides in LDL. Hydroperoxides oxidize ferrous to ferric ions in dilute acid and the resultant ferric ions are determined using ferric sensitive dyes as an indirect measure of hydroperoxide concentration. Xylenol orange [o-cresolsulfonephthalein $3^{\prime} 3^{\prime \prime}$-bis (methylimino) diacetic acid sodium salt) binds ferric ions with high selectivity to produce a coloured (blue-purple) complex with an extinction coefficient of $1.5 \times 10^{4} / \mathrm{mol}$ per $\mathrm{cm}$ and absorbance maximum of $560 \mathrm{~nm}$. The method compares favourably with the iodometric assay, TBA assay and conjugated diene measurement. No extraction step is required for analysis of lipoprotein in the $900 \mathrm{ml} / \mathrm{l}$ methanol-25 mM- $\mathrm{H}_{2} \mathrm{SO}_{4}$ environment in which the assay is performed.

Triphenylphosphine is used as a specific reductant of hydroperoxides, converting them to the corresponding alcohol. This allows the measurement of authentic hydroperoxides reacting in the assay and removes any background signal generated. Each sample is therefore measured with and without triphenylphosphine, the difference between the two being the lipid hydroperoxides in the sample. Background values for plasma have been reported to be high. The mean value obtained for native LDL is reported to be 13.3 (SD 8.8) $\mathrm{nmol} / \mathrm{mg}$ LDL (Rice-Evans et al. 1996). Lipid hydroperoxides can also be measured easily in LDL using the tri-iodide assay (El-Sadaani, 1989). The lipids of LDL are dispersed by the detergent used in an enzymic cholesterol assay kit and the hydroperoxides oxidize $\mathrm{I}^{-}$to $\mathrm{I}_{2}$ which is detected spectrophotometrically. The values given for native LDL for the iodometric method correspond to $25 \mathrm{nmol} / \mathrm{mg}$ LDL protein (El-Sadaani, 1989), $18 \cdot 6$ (SD $9 \cdot 4) \mathrm{nmol} / \mathrm{mg}$ LDL protein (Esterbauer et al. 1992) and in the range of $10-20 \mathrm{nmol} / \mathrm{mg}$ LDL protein (O'Leary et al. 1992). This sort of range was deemed to be at the borderline of the detection limit for the iodometric assay (Esterbauer $e t$ al. 1992).

The HPLC procedure for detecting lipid hydroperoxides has the advantage that the identity and mass of specific lipid peroxides may be determined down to very low concentrations, well below that available by the colorimetric methods. This sensitivity is dependent on the availability of chemiluminescence detection (Kritharides et al. 1994; Stocker et al. 1991). It has recently been reported (Kritharides et al. 1994) that LDL freshly isolated from healthy subjects was free from detectable amounts of cholesterol-ester hydroperoxides and phospholipid hydroperoxides as measured by HPLC with post-column chemiluminescence detection, suggesting that if lipid hydroperoxides are present at all, the levels must be below $1 \mathrm{nmol} / \mathrm{mg}$ LDL protein. This method of detection does require considerable dedication of technical resources and is unlikely ever to be suitable for any routine assays in large-scale clinical research. In some cases, u.v. detection can be used but with lower sensitivity. A comment should be made on the wide-ranging differences between the detected endogenous peroxide levels in LDL 
applying the HPLC-chemiluminescence method compared with the spectrophotometric assays. (A direct comparison of the methods has yet to be carried out in a single laboratory on the same LDL samples.) No one is sure whether the FOX and El-Sadaani (1989) methods are determining additional unidentified components (which might include protein hydroperoxides) or whether the HPLC assay is missing some contributing features. On the other hand, it may relate to the methods applied for isolating the LDL; for example, it has been reported that applying the FOX assay after rapid isolation procedures gives $3 \mathrm{nmol} / \mathrm{mg}$ LDL protein, compared with $13.3 \mathrm{nmol} / \mathrm{mg} \mathrm{LDL}$ using the sequential isolation methods (Kritharides et al. 1995).

\subsection{Oxidative damage to proteins}

Oxidative damage to proteins is of particular importance in vivo both in its own right (affecting the function of receptors, enzymes, transport proteins etc. and perhaps generating new antigens that provoke immune responses), and because it can contribute to secondary damage to other biomolecules, e.g. inactivation of DNA repair enzymes and loss of fidelity of DNA polymerases in replicating DNA. The chemical reactions resulting from attack of ROS or RNS on proteins are complex. Free-radical attack can generate protein peroxides, which can decompose in complex ways (Ambe \& Tappel, 1961; Fu et al. 1995).

Most use has been made of the carbonyl assay, a general assay of oxidative protein damage (Levine et al. 1995) to assess steady-state protein damage in human tissues and body fluids. The carbonyl assay is based on the fact that amino acid residues in proteins (particularly histidine, arginine, lysine and proline) are particularly susceptible to attack by ROS producing carbonyl functions. Such increased carbonyl content can be measured after reaction with 2,4-dinitrophenylhydrazine. The carbonyl assay has become widely used and many laboratories have developed individual protocols for it (Levine et al. 1994, 1995). Sometimes the assay procedures used in a particular laboratory are not precisely specified in published papers and even when they are, they often differ from those used originally by the group of Stadtman (Oliver et al. 1987; Levine et al. $1994,1995)$. This point is important because there is a considerable variation in the 'baseline' levels of protein carbonyls in certain tissues, depending on how the assay is performed (Cao \& Cutler, 1995; Lyras et al. 1996). Contrariwise, broadly comparable values for protein carbonyls in human plasma, of $<1 \mathrm{nmol} / \mathrm{mg}$ protein have been reported by most groups, so plasma protein carbonyls should be a useful marker of oxidative protein damage for nutritional studies. More work needs to be done to identify the molecular nature of the carbonyls, namely, which amino acid residues have been damaged and in which proteins they reside. Western-blotting assays based on the use of antidinitrophenylhydrazine antibodies have also been developed in an attempt to identify oxidatively-damaged proteins in tissues and body fluids (Keller et al. 1993; Levine et al. 1994). A cautionary note is the covalent binding of certain aldehyde end-products of lipid peroxidation to proteins, generating 'carbonyls'. Indeed, many oxidized molecules contain carbonyls which will interfere in the protein carbonyl assay.
Several in vitro assays for damage to specific amino acid residues in proteins have been developed including assays of 3-hydroxy-L-tyrosine (L-DOPA) (produced by tyrosine hydroxylation) (Giseg et al. 1993), valine hydroxides derived from valine hydroperoxides (Giseg et al. 1993), ring-opening products of tryptophan oxidation (Griffiths et al. 1992; Maskos et al. 1992) 8-oxohistidine (Uchida \& Kawakishi, 1993, 1994), dityrosine (Giulivi \& Davies, 1993) and ortho and meta-tyrosines, products of attachment of OH to phenylalanine (Karam et al. 1991; Wells-Knecht et al. 1993). The levels of any one (or, preferably, of more than one) of these products in proteins could, in principle, be used to assess the balance between oxidative protein damage and the repair or (more likely) hydrolytic removal of damaged proteins. The only products exploited to date have been the hydroxylated phenylalanines (Wells-Knecht et al. 1993).

Attack of various $\mathrm{RNS}\left(\mathrm{ONOO}^{-}, \mathrm{NO}_{2}^{-}\right.$and possibly some other species) on tyrosine (both free and in proteins) leads to production of 3-nitrotyrosine, which can be measured immunologically or by HPLC or GC-MS techniques (reviewed by Wells-Knecht et al. 1993). Reduction of nitrotyrosine to aminotyrosine increases the sensitivity of measurement, since the latter compound can be measured using highly-sensitive electrochemical detection. Nitrotyrosine is also excreted in human urine (Oshima et al. 1990), although the possible confounding effect of dietary nitrotyrosine (if any) and of dietary nitrate and/or nitrite is yet to be evaluated.

For measures of total ongoing protein damage, urinary nitrotyrosine (Oshima et al. 1990) might be useful as a generalized index of attack by RNS. Very little research has been carried out on the presence of oxidized amino acids and their metabolites in urine, except that bityrosine has been detected and can be measured by HPLC with fluorescence detection. More work needs to be done in this area, and the possible confounding effects of oxidized proteins and amino acids in the diet (e.g. in irradiated foods; Halliwell \& Chirico, 1993) must be considered.

\subsection{Measurement of antioxidant nutrients, carotenoids and flavonoids extracted from human plasma}

Several antioxidants are routinely measured in plasma: $\alpha$ tocopherol (and $\gamma$-tocopherol) whose antioxidant roles are well clarified in vivo; $\beta$-carotene, lycopene and other dietary carotenoids for which there is, as yet, little evidence of antioxidant activity in vivo; ascorbic acid, the most efficient reducing agent in vivo, its redox potential defining its central role as an aqueous phase antioxidant. There is, as yet, little information as to the importance of dietary flavonoids as antioxidants in vivo, nor evidence for such activity in vivo, although these polyphenols are highly efficacious freeradical scavengers in vitro. Furthermore, it is only recently that it has become possible to detect and identify flavonoids (and other glycosides) in human plasma, in non-supplemented subjects.

Table 1 indicates the most favourable systems for detection, identification and quantification of the antioxidants by HPLC. Plasma samples can be stored at $-70^{\circ}$. 
Table 1. The most favourable systems for detection, identification and quantitation of antioxidants by HPLC

\begin{tabular}{|c|c|c|}
\hline Antioxidant & Method & Conditions \\
\hline$\alpha$-Tocopherol and $\gamma$-tocopherol & $\begin{array}{l}\text { HPLC with fluorescence detection } \\
\qquad\left(\lambda_{e x} 296 \lambda_{e m} 340\right)\end{array}$ & $\begin{array}{l}\text { Solvent system: hexane-methyl-t-butyl ether }(92: 8, \mathrm{v} / \mathrm{v}) \\
\text { Column: Novapak Silica } 150 \times 4.6 \mathrm{~mm}(4 \mu \mathrm{m}) \\
\text { Internal standard: } \delta \text {-tocopherol } \\
\text { Detection limit: in plasma, } 1 \cdot 1 \mu \mathrm{mol} / /\end{array}$ \\
\hline Carotenoids & HPLC with diode array detection & $\begin{array}{l}\text { Solvent system: acetonitrile-methanol }(90: 10, \mathrm{v} / \mathrm{v}) \\
\text { Column: Supelco PKB } 100,5 \mu \mathrm{m} \\
\text { or } \\
\text { Solvent system: acetonitrile-methanol-dichloromethane- } \\
\text { hexane (gradient) } \\
\text { Column: Merck lichrocart man-fix, } 5 \mu \mathrm{m} \\
\text { Detection at: } 455 \mathrm{~nm} \text { ( } \beta \text {-cryptoxanthin) } \\
\qquad \begin{aligned} 468 \mathrm{~nm} \text { (canthaxanthin) } \\
474 \mathrm{~nm} \text { (lycopene) } \\
454 \mathrm{~nm} \text { ( } \beta \text {-carotene) }\end{aligned}\end{array}$ \\
\hline Flavonoids & HPLC with diode array detection & $\begin{array}{l}\text { Solvent system: } 1 \mathrm{ml} / / \mathrm{HCl} \text { in } 20 \% \text { of aqueous } \\
\text { methanol and acetonitrile (gradient) } \\
\text { Column: Nova-Pak C18 } 250 \times 4.6 \mathrm{~mm}(4 \mu \mathrm{m}) \\
\text { Internal standard: salicylic acid } \\
\text { Detection: } 280 \mathrm{~nm} \text { (selective) }\end{array}$ \\
\hline
\end{tabular}

$\lambda_{e x}$ and $\lambda_{e m}$, excitation and emission wavelengths.

\subsection{Conclusions}

There is still a long way to go until validated, precise biomarkers of oxidative damage become routinely available.

Concerning oxidative damage to DNA, both HPLC and $\mathrm{GC}-\mathrm{MS}$ have their relative merits but also their limitations. Sight must not be lost of the potential for oxidative damage to DNA during its isolation from cells and tissues. Nevertheless, artifacts arising during derivatization are also problematical and HPLC with electrochemical detection is one proposed route which circumvents the need for derivatization. Liquid chromatography-mass spectrometry techniques are also under development. An initiative by the British Ministry of Agriculture Fisheries and Food (MAFF) is seeking to coordinate, within the MAFF Antioxidants in Foods Research Programme, validation work at a number of centres within Europe, concerned with biomarkers of oxidative damage to DNA. The agenda seeks to have validated agreed biomarkers available for a new generation of human studies within 2-3 years.

With regard to ex vivo markers of lipid oxidation in vivo, the measurement of circulating isoprostanes and lipid hydroperoxides is the best approach for plasma, the latter applying HPLC with chemiluminescence detection. Where this detection system is not available, the HPLC-TBA method has been shown to be an alternative, although lacking the high precision of the former. While the principle of isoprostane analysis is highly promising, this is still in its early infancy as there is a dearth of information in the peerreviewed literature as yet, but it is rapidly gaining momentum. For urinary markers of lipid oxidation as an indicator of whole-body lipid peroxidation, the development of the isoprostane analytical techniques will be an important advance, since hydrocarbon gas exhalation has too many confounding variables to be applicable to studies on freeliving human subjects.

The potential for the application of methods for ex vivo detection of in vivo protein oxidation is promising. The outcome, of course, is the balance between damage and repair but to date very few products of oxidative damage to proteins have been exploited in this context.

There is also a lot of excitement and activity in the areas of evidence for the formation of RNS in vivo. A major stumbling block here in the accumulation of evidence for oxidative damage in vivo is the confounding effects of the presence of oxidized proteins and amino acids in the diet. A lot more work needs to be done in this area.

\section{Nutritional options modulating oxidative damage and antioxidant defence systems}

\subsection{Introduction}

The body's antioxidant defence system is capable of being altered by dietary means. A first strategy to balance oxidative damage and antioxidant defence of human cells and tissues would be to enhance the antioxidant capacity by optimizing the dietary intake of antioxidants. A second approach may be to neutralize oxidative compounds in the diet. Crucial to these strategies is knowledge of the required level of relevant antioxidants in the diet to provide protective effects. Another prerequisite is accurate information about food sources, content and bioavailability of antioxidants.

Epidemiological studies are necessary to quantify the impact of antioxidants on disease aetiology. Intervention trials formally test the efficacy of enhancing intake of antioxidants. In evaluating these health benefits preferably hard end-points (disease incidence, or recurrence and mortality) should be used. Alternatively intermediate endpoints may be effective, provided that they are genuinely predictors of the disease of interest. In research on functional foods, the development and application of biomarkers is extremely important. In the causal pathway of disease occurrence one can distinguish biomarkers of exposure (dietary intake), biomarkers of biological response and of (subclinical) disease, and biomarkers of susceptibility. For antioxidants, all types of markers have clear relevance. For example, blood levels of vitamin $E$ (an 
exposure marker) may be studied in relation to oxidation resistance of LDL (a biological response marker) or to carotid artery wall thickness (a disease marker), in subjects with familial hypercholesterolaemia, or specific genotype (both susceptibility markers). Although biomarkers have the potential for improving validity and reducing bias, several problems are encountered. Biomarkers of exposure should accurately reflect relevant dietary intake or body status and early disease markers should have predictive value for the hard end-point. Since chronic diseases have long latency periods requiring large initial numbers to evaluate health effects, biomarkers of intermediate end-points may, in certain circumstances, legitimately be used more efficiently.

An effective nutritional strategy will require knowledge of the type of antioxidants in the diet, their food sources, bioavailability and required levels of intake for protective effects. Protective effects of antioxidants have been found in mechanistic studies in vitro and in vivo, and epidemiological studies and certain intervention studies have provided useful information. However, it is appropriate to consider the totality of the evidence from basic science, epidemiology and intervention studies, rather than to rely on the evidence from any one type of study.

\subsection{Dietary antioxidants}

4.2.1. Sources of dietary antioxidants. There are both nutrient and non-nutrient antioxidants. Non-nutrient antioxidants include flavonoids (found for example in tea, red wine, onions, and apples), polyphenols and terpenes. The focus here will be on nutrient antioxidants, in particular vitamin $\mathrm{C}$, vitamin $\mathrm{E}$ and carotenoids, although possible effects of non-nutrient antioxidants must be borne in mind in reaching complete understanding. Thus, it is important to ascertain whether non-nutrient antioxidants are genuinely bioavailable, in the particular sense of whether they are delivered under normal circumstances to tissues where they might be expected to be effective. Their activity as antioxidants themselves, or whether they participate in a cyclical fashion with other antioxidants, also remains to be established.

The flavonoid content of beverages has been of great interest recently (Hertog et al. 1993). There is a large variation in the quercetin and myricetin content of red wines, which appears to depend on the type of grape and the vineyard of origin. Quercetin, kaempferol and myricetin are present in black and green teas, so that, together with fruit juices (mainly quercetin), these beverages can provide substantial amounts of flavonoids in the human diet.

Vitamin C is found in citrus fruits, peppers, potatoes and other fruits and vegetables. The principal sources of vitamin $E$ are vegetable oils and wheat germ. Other sources are nuts, seeds and leafy green vegetables. Of the over 600 carotenoids, $\beta$-carotene has been the most extensively studied. Good sources of $\beta$-carotene are yellow or orange fruits and vegetables such as carrots, sweet potatoes, apricots, and mangoes, as well as dark green leafy vegetables such as spinach. There is now increasing interest in other major dietary carotenoids including lycopene (tomatoes), lutein (spinach, broccoli, maize), zeaxanthin (maize), $\alpha$-carotene (carrots), and $\beta$-cryptoxanthin (citrus fruits). Chemical analysis of food products is steadily improving and until recently, there was a lack of reliable data for the food content of carotenoids other than $\beta$-carotene. However, the recently released carotenoid food composition database of the US Department of Agriculture has included analysis of $\alpha$-carotene, $\beta$-cryptoxanthin, lutein, zeaxanthin, and lycopene. A number of epidemiological studies of $\beta$-carotene and risk reduction of certain diseases are now being re-evaluated using information derived from this source on the other carotenoids.

4.2.2. Antioxidant intake and status. Several crosssectional surveys in a variety of population groups have been conducted. From these studies it can be concluded that according to the recommended daily allowances (RDA), the intake of antioxidants is adequate in healthy subjects. Lower levels have been observed in smokers, the elderly, and in patients with specific diseases or risk factors and several studies have demonstrated that intake of a number of antioxidants may be suboptimal in certain populations. In countries such as France and Italy antioxidant intake is largely adequate due to the abundant supply of fresh fruits and vegetables. However, in other countries where the selection of products is limited or more seasonal, a number of population groups are not able to meet the minimum requirements for vitamins $\mathrm{E}, \mathrm{C}$ or $\beta$-carotene. At particular risk are the less affluent and the elderly.

The RDA, which are not established for carotenoids, are defined to prevent nutrient deficiencies and do not take into account the reduction in risk of chronic diseases. The levels of antioxidant nutrients that are effective in the reduction in risk of chronic diseases generally lie higher than the RDA. Since antioxidants may play important roles in the prevention of chronic diseases, the question is what would be the optimal range of intake to recommend. Lachance (1996) distinguishes the following categories: 'experimental protective intake' 'amount in optimal menus', 'desirable blood level', 'calculated intake necessary to achieve desirable blood levels'. For example, for vitamin E (current RDA: $10 \mathrm{mg}$ men, $8 \mathrm{mg}$ women) the following values have been proposed: experimental protective intake $>23-100 \mathrm{mg}$; amount in optimal menus $23 \mathrm{mg}$; desirable blood level $23 \mu \mathrm{mol} / \mathrm{l}$; calculated intake necessary to achieve desirable blood levels $23 \mathrm{mg}$. For carotenoids the following values have been suggested: $>4 \mathrm{mg}, 5.7 \mathrm{mg}, 0.4 \mu \mathrm{mol} / /, 3.2 \mathrm{mg}$ respectively. Diplock (1994) reviewed epidemiological studies of antioxidants and disease and suggested that the following daily intakes were associated with a reduced risk of cancer and cardiovascular disease; $150 \mathrm{mg}$ vitamin $\mathrm{C}$, $40-60 \mathrm{mg}$ vitamin $\mathrm{E}$, and $9-12 \mathrm{mg} \beta$-carotene. Lachance (1996) has estimated optimal daily antioxidant intakes to be $145 \mathrm{mg}$ vitamin $\mathrm{C}, 23 \mathrm{mg}$ vitamin $\mathrm{E}$, and $3.2 \mathrm{mg}$ carotene. Biomarkers of antioxidant intake reflecting internal status (blood, adipose tissue, nails etc.) have been used successfully but need much further development, and these also need to be non-invasive. Conceptually it is important to know the biological relevance and exposure timeframe of the biomarker. It is not only the dietary intake but other exogenous factors such as smoking and alcohol intake, and endogenous factors, that affect antioxidant status measured by a biomarker. For many bio-active compounds intake data are hard to get and, thus, more reliance will have to be 
placed on biomarker data. It is thus imperative that the biomarkers that are used can be shown to be relevant to the outcome of the balance between oxidant and antioxidant in the majority of the population.

4.2.3. Bioavailability of antioxidants. Bioavailability of antioxidants depends on several food and host-related factors, as recently summarized by de Pee \& West (1996) for carotenoids. A well-recognized food-related factor is the amount of antioxidant in a meal; for nutrients which are absorbed by a process of passive diffusion, the proportion of antioxidant absorbed decreases with increasing amounts in the food. The molecular forms of antioxidants in foods, for example, for which isomers or molecular linkages such as esters exist, are also important determinants of bioavailability. In addition, the food matrix in which antioxidants are located often influences availability of the nutrient. For example, $\beta$-carotene is organized in a pigment-protein complex in green vegetables, but found in lipid droplets in other vegetables and fruits. The $\beta$-carotene can be released more readily, and is thus more biovailable, from a fat droplet than from a protein complex.

Host-related factors influencing bioavailability include genetic factors, nutrient status and absorption modifiers. Absorption modifiers for fat-soluble vitamins and carotenoids, are lipids in the diet. To ensure efficient absorption, sufficient fat must be present in the meal and diet.

A recent epidemiological study on lycopene intake and prostate cancer illustrates the importance of bioavailability in functional food research. Intake of tomatoes, tomato sauce, and pizza were significantly related to lower risk of prostate cancer. Tomato juice was not associated with a protective effect (Giovannucci et al. 1995). The lycopene from tomato juice has a low bioavailability, but cooking tomatoes in an oil-based medium substantially enhances intestinal absorption.

4.2.4. Fat intake and antioxidant status. Consumption of reduced-fat products in order to reduce energy intake, or of products with enhanced PUFA content (Sarkkinen et al. 1993), may also affect the antioxidant status. It was shown that vitamin $\mathrm{E}$ intake was significantly lower among subjects who had an increased intake of reduced-fat products; however, no effect was observed for $\beta$-carotene or lycopene, or for the antioxidant enzyme activities (superoxide dismutase, catalase and glutathione peroxidase) (Velthuis-te Wierik et al. 1996). There is, however, evidence that plasma antioxidant levels may not be a reliable index of body status; it was found that erythrocyte levels were unaffected in a study in men who had significantly lowered plasma levels of vitamin E (Haddad \& Blankenship, 1985). Inhibitors of dietary fat absorption, which may be used to counteract obesity, appear to have fatsoluble vitamin-lowering properties (Melia et al. 1996). Besides a reduced antioxidant intake, fat substitutes like the sucrose polyester-based products may also reduce the absorption of fat-soluble antioxidants, although this only occurs when the fat intake is below $20 \%$ of total energy. However, published data do not suggest a major impact on the absorption of antioxidants when the level of inclusion of the fat substitute in the diet is low, such as would be the case when snack foods are prepared using the fat substitute. In general, diets low in PUFA do result in lower intake of vitamin $\mathrm{E}$. On the other hand, PUFA-rich diets may affect the antioxidant-prooxidant balance requiring higher intake of antioxidants.

\subsection{Epidemiological studies on protective effects of antioxidants}

4.3.1. Cardiovascular disease. Several scientific reviews have addressed the role of antioxidants in cardiovascular disease (van Poppel et al. 1994). As discussed earlier, the primary role of antioxidants in reducing the risk of cardiovascular disease is through inhibition of peroxidation in LDL, although they may also influence other cardiovascular disease processes.

The epidemiological evidence for a protective role of antioxidants in cardiovascular disease is strongest for vitamin E. Three large-scale epidemiological studies demonstrated a relation between vitamin $\mathrm{E}$ intake and CHD. The Nurses Health Study, conducted in 87245 women, found a significant $34 \%$ reduction in CHD in women who had consumed vitamin $\mathrm{E}$ supplements containing more than $67 \alpha$-tocopherol equivalents ( $\alpha$-TE) daily for more than 2 years (for a definition of $\alpha$-tocopherol equivalents see p. S95). The Health Professionals Study of 39910 men showed vitamin $E$ to be associated with a $41 \%$ reduction in risk of CHD. Again, the greatest risk reduction was found with intakes of supplements of more than $67 \alpha-$ TE for 2 years or more.

Kushi et al. (1996) reported a significant $62 \%$ reduction in mortality from CHD in women consuming foods containing more than $6.46 \alpha-\mathrm{TE} / \mathrm{d}$. Surprisingly, there was no risk reduction seen with use of vitamin $E$ supplements. This may be due to the lack of information on duration of supplement use, since from the other studies it appears that benefits of supplements are evident only after 2 years.

Several epidemiological studies have examined the association between vitamin $\mathrm{C}$ and cardiovascular disease. A European cross-cultural study found a significant inverse relationship between CHD mortality and serum vitamin C levels. The first National Health and Nutrition Examination Survey (NHANES I) results showed a $50 \%$ reduction in cardiovascular mortality associated with a daily consumption of greater than $50 \mathrm{mg}$ vitamin C. Gale et al. (1995) reported a significant protective effect against stroke of vitamin $\mathrm{C}$ intakes more than $45 \mathrm{mg}$. However, neither the Nurses' Health Study nor the Health Professionals Study described earlier found a significant protective effect of vitamin $\mathrm{C}$.

A recent study found that use of both vitamin $E$ and $C$ supplements was significantly more protective against cardiovascular mortality than use of vitamin $\mathrm{E}$ supplements alone, or no use of supplements (Losonczy et al. 1996).

The epidemiological evidence for a role of carotenoids in cardiovascular disease prevention has been reviewed by Kohlmeier \& Hastings (1995). A number of studies consistently showed a decreased risk of CHD among subjects with high $\beta$-carotene intake or serum levels. In the Massachusetts Health Care Panel study of 1299 elderly people, Gaziano et al. (1995) found a $75 \%$ reduction in risk of fatal myocardial infarction in subjects in the highest quartile of 
carotene intake. A study of 25802 subjects showed a significant doubling of the risk of myocardial infarction in subjects with low serum $\beta$-carotene levels (Street $e t$ al. 1994). Several studies have, however, shown increased risk of $\mathrm{CHD}$ among subjects with low $\beta$-carotene status. The risk seems to be confined to current smokers. However, $\beta$ carotene intake contributes about $25 \%$ of total carotenoid intake, thus carotenoids other than $\beta$-carotene as well as other components in fruits and vegetables may be responsible. The sparse data on individual carotenoids do not allow any firm conclusions. Several non-nutritive bioactive compounds may be of relevance in the aetiology of cardiovascular disease. Epidemiological studies on flavonoids are promising, but evidence of benefit is still fragmentary (Hertog, 1994; Muldoon \& Kritchevsky, 1996).

4.3.2. Cancer. Numerous epidemiological studies have shown that individuals who regularly consume fruits and vegetables have a decreased risk of cancer. A protective effect of fruit and vegetable consumption was found in 128 of 156 dietary studies (Block et al. 1992). High intake of fruits and vegetables (in the upper one-fourth of the population) is associated with an approximately $50 \%$ reduced risk of cancer, depending on the tumour site. The most consistent evidence of risk reduction associated with fruits and vegetables has been seen in the epithelial cancers of the respiratory and gastrointestinal tract (Steinmetz \& Potter, 1991). Evidence is strong for lung cancer, with significant risk reduction found in twenty-four of twentyfive studies, as well as for stomach and pancreatic cancer with protection in twenty-six of thirty studies. For oesophageal, laryngeal and oral cancer, twenty-eight of twenty-nine studies showed a significantly reduced risk with fruit consumption. Cancers of the cervix, ovary and endometrium were associated with a significant risk reduction in eleven of thirteen studies.

Although there is, therefore, indirect evidence that antioxidants (carotenoids, vitamin $\mathrm{C}$, vitamin $\mathrm{E}$ and possibly non-nutritional antioxidants) may be beneficial (TaylorMayne, 1996), it has to be shown whether, and to what extent, this is true for individual antioxidants and which specific components are responsible. To illustrate the complexities: plasma $\beta$-carotene in the normal physiological range is inversely related to lung cancer incidence. Other carotenoids such as $\alpha$-carotene, which is strongly correlated with $\beta$-carotene, may however be the relevant factor. In addition, other bioactive components in fruits and vegetables or other aspects of lifestyle, including diet, may play a causal role. Focusing, in epidemiological studies, on food items which mainly contribute to the daily intake of a specific bioactive compound, e.g. tomatoes and tomato products in the case of lycopene, may be a promising research strategy to identify the responsible factor.

In attempts to identify the components in fruits and vegetables associated with risk reduction of cancer, epidemiological studies focusing on intake or serum levels of specified antioxidants have been undertaken. In epidemiological studies of the protective effect of vitamin $\mathrm{E}$ on various cancer sites, vitamin $\mathrm{E}$ was associated with slightly reduced risk of lung cancer in studies involving low exposure to tobacco smoke. Several studies of oral, pharyngeal and cervical cancer have also found a relationship between vitamin $\mathrm{E}$ status and cancer risk. The evidence for stomach and pancreatic cancers has not been consistent, and no association with breast cancer has been found. Diets high in vitamin $E$ intake have been less consistently shown to be associated with cancer protection. Moreover, observational studies of vitamins $\mathrm{C}$ and $\mathrm{E}$ consumed in supplements provide little support for a strong protective role against cancer.

As discussed earlier, vitamin $\mathrm{C}$ inhibits the formation of carcinogenic nitrosamines, stimulates the immune system, protects against chromosomal breakage, and regenerates vitamin $\mathrm{E}$ as part of the antioxidant defence system. The epidemiological evidence for a risk-reducing role of vitamin $\mathrm{C}$ in cancer is not as strong as for fruits and vegetables. However, an extremely strong and consistent protective effect of vitamin $\mathrm{C}$ was found in seventeen of nineteen studies of stomach, oesophageal, oral and pharyngeal cancers (Block et al. 1992). Additional studies showed that subjects with low serum levels of vitamin $\mathrm{C}$ have a $50 \%$ increased risk of gastric metaplasia or chronic gastritis, which are both precancerous lesions.

The most consistent body of epidemiological evidence showing protective effects of an antioxidant nutrient on cancer is for $\beta$-carotene. The strongest results are seen for lung cancer. Of twenty-five studies which investigated $\beta$ carotene and lung cancer rișk, twenty-four showed a significant reduction in risk with high $\beta$-carotene intakes or plasma levels (van Poppel \& Goldbohm, 1995). For stomach cancer, of fifteen studies, eight found a significant risk reduction associated with $\beta$-carotene, and six showed a non-significant risk reduction. Only one study did not find a protective effect of $\beta$-carotene against stomach cancer. No consistent associations were found with colorectal, prostate or breast cancer.

4.3.3. Other age-related diseases. Cataract and AMD are eye disorders which show increasing incidence among the elderly. Cataracts result from glycosidation of lens proteins initiated by u.v. light, which leads to opacification of the lens. It has been shown that in vitro lens proteins may be protected against oxidative attack by carotenoids and vitamins $\mathrm{C}$ and $\mathrm{E}$. Of ten epidemiological studies, nine showed strong inverse relationships with at least one antioxidant nutrient. Observations are most consistent for vitamins $\mathrm{C}$ and $\mathrm{E}$.

Macular degeneration is the leading cause of irreversible blindness among persons older than 65 years. The carotenoids lutein and zeaxanthin are present in the retina as pigments to protect against the damaging effects of light; strong inverse relationships were found between intakes of $\beta$-carotene, lutein and zeaxanthin and risk of AMD. Consumption of spinach, which is a good source of lutein and zeaxanthin, was also associated with significantly reduced risk of AMD.

Free radicals have also been implicated in the development of neurodegenerative disorders such as Parkinson's disease and Alzheimer's disease, and in diabetes, rheumatoid arthritis, and chronic obstructive pulmonary diseases. These age-related diseases may, therefore, be beneficially influenced by antioxidant consumption. However, as yet few epidemiological data exist on the association of antioxidants with these disease risks. 
The question whether it is a specific antioxidant or another component, or mixture, of fruits and vegetables that exerts the protective effect on disease cannot be definitively answered by epidemiological studies. Additional information provided by intervention trials is needed before drawing any final conclusions.

\subsection{Human intervention studies of antioxidants}

The gold standard for testing the effectiveness of specific antioxidants is a randomized placebo-controlled intervention trial, preferably with a hard end-point such as disease occurrence or cause-specific mortality. Since trials conducted with clinical end-points of disease incidence or mortality are exceedingly costly and time consuming, intermediate end-points or validated biomarkers are increasingly being used. The limitations of intervention trials are that they can often only be interpreted for the particular study population, and for the antioxidant dose provided during the trial. Most of the intervention trials have focused on cardiovascular disease and cancer; only a few studies have addressed other age-related disorders, i.e. cataract.

4.4.1. Cardiovascular disease. Intermediate endpoints. A number of studies have supplemented individuals with antioxidants and measured the reduction in lipid peroxidation. However, indices of lipid peroxidation have not yet been definitively associated with cardiovascular disease end-points. The only intervention studies which have evaluated the effect of antioxidants on cardiovascular disease using intermediate disease endpoints have been with vitamin $\mathrm{E}$. Using arterial narrowing as a marker for cardiovascular disease progression, the effect of vitamin $E$ was evaluated in 100 patients randomized to $804 \alpha$-TE or placebo following angioplasty. The group receiving vitamin $\mathrm{E}$ showed significantly less recurrence of stenosis over the 4-month supplementation period.

Carotid artery wall thickness, measured by non-invasive arterial wall imaging, has also been used as a reliable intermediate end-point for atherosclerosis. A recent study (Azen et al. 1996) demonstrated that use of vitamin $\mathrm{E}$ supplements larger than $67 \alpha$-TE/d reduced the progression of atherosclerosis.

Disease end-points. Although vitamin E shows promise in reducing cardiovascular disease, relatively few intervention trials have been conducted. The first of the trials to be reported was the $\alpha$-tocopherol $\beta$-carotene (ATBC) study which found no effect on cardiovascular disease mortality among heavy chronic smokers receiving $50 \mathrm{mg}$ vitamin $\mathrm{E}$ daily (Albanes et al. 1994).

Linxian, China was the site of a study which gave a multivitamin-multimineral supplement plus $15 \mathrm{mg} \beta$-carotene and found a $38 \%$ reduction in stroke incidence in subjects with oesophageal dysplasia. A non-significant $10 \%$ reduction in stroke incidence was also observed in a trial involving 35000 subjects from the general population of Linxian who received $15 \mathrm{mg} \beta$-carotene, $30 \mathrm{mg}$ vitamin $\mathrm{E}$ and $50 \mu \mathrm{g}$ Se (Blot et al. 1993).

A trial conducted in 161 Japanese subjects, who consumed 3 or $100 \mathrm{mg} \alpha$-tocopherol/d for 6 years, showed a significant reduction in the number of coronary disorders in the $100 \mathrm{mg} / \mathrm{d}$ group (Takamatsu et al. 1995). The recently published Cambridge Heart Antioxidant Study (CHAOS) found a significant reduction in the incidence of non-fatal myocardial infarctions in 2002 subjects randomized to 268 or $536 \alpha$-TE/d (Stephens et al. 1996). However, there was a non-significant excess of cardiovascular deaths in the vitamin E-supplemented group.

$\beta$-Carotene supplementation resulted in increased cardiovascular disease incidence or mortality in two studies conducted in high-risk populations of smokers and asbestos-exposed workers, ATBC and the $\beta$-carotene and retinol efficacy trial (CARET) (Albanes et al. 1994; Omenn et al. 1996). In a trial of healthy male US doctors, the Physicians' Health Study (Hennekens et al. 1996) showed no effect on cardiovascular disease following 12 years of supplementation with $50 \mathrm{mg} \beta$-carotene every second day. This was in contrast to earlier findings in a subgroup of the study which found a significant $50 \%$ reduction in secondary coronary events in subjects randomized to $\beta$-carotene (Gaziano et al. 1995).

It is known that in vitro vitamin $\mathrm{C}$ can regenerate vitamin $E$ from the vitamin $E$ radical which is formed during the inhibition of lipid peroxidation. Several large-scale trials are currently underway to study a combination of vitamins $E$ and $\mathrm{C}$, and $\beta$-carotene, in the prevention, or amelioration, of risk of cardiovascular disease.

4.4.2. Cancer. Intermediate end-points. Most of the intervention trials investigating the potential role of antioxidants in reducing the risk of cancer have focused on precancerous lesions. Relatively little work has been done on biomarkers for cancer, such as DNA damage. Recent reviews have summarized the use of antioxidants in oral leukoplakia, a precancerous lesion of the oral cavity (Kaugars et al. 1994; Garewal, 1995). Significant impact on lesion size or occurrence was found in seven intervention studies with a supplement of between $30 \mathrm{mg}$ and $180 \mathrm{mg} \beta$ carotene. $\beta$-Carotene has also been shown to reduce cervical as well as gastric dysplasia, and there are a number of intervention studies currently in progress.

Several promising biomarker candidates for cancer have been suggested. Ideal characteristics of these biomarkers include: they should appear earlier than, or more frequently before, tumour development; they should be directly associated with tumour progression; they should be shown to be reversible; they should have inexpensive, accurate and simple methods of detection; they should be validated. Biomarkers of cellular proliferation and differentiation e.g. colonic adenomatous polyps and premalignant lesions such as dysplasia have been used to evaluate the preventive role of nutrient antioxidants. In contrast to observations using microscopic measures of cellular proliferation, the major antioxidants vitamins $E$ and $C$, and $\beta$-carotene seem to be ineffective in decreasing the recurrence of colonic adenomas. Clarification is needed of changes in biomarkers that have been shown to occur with progression of proliferation, and in subsequent steps in the carcinogenic process, before definite conclusions can be drawn from these intermediate biomarker data.

The preventive potential of antioxidants has been tested with intermediate end-points for other tumour sites, such as 
lung, mouth, oral cavity and cervix. It is difficult to summarize the overall findings. However, until now, no really strong benefits have been identified. There is a need for a second generation of chemoprevention trials that include biomarkers, provided that these markers have fulfilled the criteria described earlier.

Disease end-point. There have been a number of intervention trials which studied the potential for $\beta$-carotene to reduce the risk of cancer. The 5-year Linxian trial of 29584 adults in China found significant reductions in mortality from total and stomach cancer of $13 \%$ and $21 \%$ respectively, in the group randomized to $15 \mathrm{mg} \beta$-carotene, $30 \mathrm{mg}$ vitamin $\mathrm{E}$ and $50 \mu \mathrm{g}$ Se (Blot et al. 1993). In 3318 adults with oesophageal dysplasia in the same community, there was a non-significant $8 \%$ decrease in oesophageal cancer in subjects randomized to a multivitamin, multimineral supplement with $15 \mathrm{mg} \beta$-carotene.

The Finnish ATBC study of 29133 chronic heavy smokers tested the effects of $20 \mathrm{mg} \beta$-carotene, either alone or in combination with $33.5 \alpha$-TE for an average of 6 years. There was a significant increase of lung cancer incidence $(16 \%)$ in the groups which received $\beta$-carotene (ATBC Cancer Prevention Study Group, 1994). A more detailed analysis of the results revealed that the increased risk of lung cancer appeared to be restricted to participants who had smoked more than twenty cigarettes daily over an average period of 30 years (Albanes et al. 1996).

The CARET study of 18314 subjects at high risk of lung cancer (heavy smokers, and asbestos-exposed workers) evaluated the combination of $30 \mathrm{mg} \beta$-carotene and 25000 IU vitamin A (equivalent to $7.5 \mathrm{mg}$ retinol) over an average of 4 years. The intervention group had a significantly increased risk of lung cancer (relative risk 1.36; Omenn et al. 1996). A reduced risk of lung cancer (relative risk $0 \cdot 80$ ) was seen in subjects who were former smokers at the beginning of the study. Interestingly, participants with high initial serum $\beta$-carotene concentrations had a $31 \%$ reduction in risk of lung cancer $(P=0.003)$, regardless of which group they were randomized to. This effect was also seen in the ATBC study and Physicians' Health Study which was conducted over 12 years in 22071 male physicians who consumed $50 \mathrm{mg} \beta$-carotene every second day. There was no beneficial influence of $\beta$-carotene on cancer incidence. However, due to the long duration of the trial it is important to note that there were also no negative effects seen (TaylorMayne, 1996).

\subsection{Conclusions}

Epidemiological studies support the hypothesis that the antioxidants vitamin $\mathrm{E}$, vitamin $\mathrm{C}$ and $\beta$-carotene may play a beneficial role in reducing the risk of several chronic disorders. More research is needed on the impact of other non-nutrient compounds, such as other carotenoids and flavonoids, on human health.

Human intervention trials testing the efficacy of antioxidants do not allow firm conclusions because of inconsistent findings, an insufficient number of studies and the use of varying doses. However, there is some evidence that large doses of $\beta$-carotene may be deleterious to the health of heavy habitual smokers.
In general, human intervention studies using hard endpoints should be regarded as the gold standard. However, for diseases with a long induction period, such as cancer and cardiovascular disease, these types of studies may not be very feasible because of high costs, and intermediate endpoints need to be sought to overcome this difficulty. The relationship between the disease and the nutritional factors that may have been involved at an early stage of its aetiology is, however, very complex and difficult to interpret. The development of biomarkers may, however, help in understanding the complexity of degenerative diseases at their different stages.

\section{Potential safety implications related to antioxidant nutritional enhancement}

\subsection{Introduction}

The substances to be considered are the antioxidant nutrients vitamin $\mathrm{C}$ (ascorbic acid), vitamin $\mathrm{E}$ ( $\alpha$-tocopherol): the carotenoids (particularly $\beta$-carotene): and the non-nutrient antioxidants, mainly flavonoids, which occur in food and which may be significant in the overall antioxidative protection afforded by the diet. This benefit may be conferred in three main ways: (1) as antioxidants in food during storage and in the gastrointestinal tract; (2) as antioxidants in the human body in vivo; (3) by providing protection in food against oxidation as well as acting as true antioxidants in vivo. From the point of view of safety, the effect of antioxidants must strictly be concerned with their effects in vivo; however, the possibility that a given compound may be converted by chemical reaction, or bacterial action, into a toxic substance within the gastrointestinal tract, or during storage of the food that contains it, must also be borne in mind because such products may themselves be absorbed and exert their toxicity in vivo.

\subsection{Vitamin $C$}

The tolerance and safety of ingested vitamin $\mathrm{C}$ in human subjects has been reviewed several times (Hanck, 1982; Rivers, 1989; Diplock, 1995). In their review Bendich \& Langseth (1995) consider in detail the fourteen controlled clinical studies that have reported no side-effects of vitamin $\mathrm{C}$ dosage, consistent with uncontrolled anecdotal reports which have appeared. In a more recent detailed review (Bendich, 1997), twenty-two placebo-controlled doubleblind studies are reviewed that indicate no consistent detrimental side-effects of dosages of vitamin $\mathrm{C}$ up to daily doses of $6 \mathrm{~g}$. This conclusion is supported by the findings of a further eight less-well-controlled studies. The fact that very large numbers of people regularly take large doses of vitamin $\mathrm{C}$ without reports of any adverse effects is anecdotal support for the view that vitamin $C$ is very safe and free from any adverse side-effects. It is clear that overload with vitamin $C$ cannot occur in man even at very high levels of dietary intake (Rivers, 1989). Absorption, tissue concentration, metabolic pathways in which ascorbate participates and renal elimination are all controlled by homeostatic mechanisms. The amount of a dose of vitamin $\mathrm{C}$ that is absorbed is inversely proportional to the size of the 
dose and saturation was achieved at a $K_{\mathrm{m}}$ of $5.44 \mathrm{~mm}$ in a human study using intestinal perfusion. A consistent body pool size of ascorbate in man of about $20 \mathrm{mg} / \mathrm{kg}$ body weight, which appeared to change little irrespective of increases in intake to very high levels, has been reported (Kallner et al. 1979). However, a recent detailed study (Levine et al. 1996) provides more information; steadystate plasma concentrations were determined in normal subjects following administration of $30-2500 \mathrm{mg}$ vitamin C daily. Steady-state plasma concentrations followed sigmoid kinetics, the steep portion of the curve occurring between 30 and $100 \mathrm{mg} / \mathrm{d}$, and complete saturation did not occur until a daily intake of $1000 \mathrm{mg}$. Different kinetics were obtained in blood cells.

Possible adverse effects on human health have been reported from time to time. However, an exhaustive search of the literature has failed to find confirmation of this, and in each case evidence exists which refutes the finding which has led to the suggestion. This is summarized as follows. (1) The formation of urinary oxalate stones in subjects ingesting large amounts of vitamin $\mathrm{C}$ over a long period proved to be without foundation. Although human subjects do metabolize some ascorbate to $\mathrm{CO}_{2}$, they excrete considerable amounts of unchanged ascorbate, and a range of metabolites among which is a small amount of oxalate; intake of ascorbate at levels in excess of the level required to maintain plasma levels at about $10 \mathrm{mg} / 1$ results in excretion of the excess ascorbate unchanged (Kallner et al. 1979). Approximately $35-40 \%$ of the daily excretion of oxalate is derived from ascorbate, but ingestion of large amounts of vitamin $C$ results in a very small increase in the excretion of oxalate. It was shown clearly (Schmidt et al. 1981) that there is no dose-response relationship between administered vitamin $\mathrm{C}$ and excreted oxalate. Part of the explanation of the difference between this result and that of earlier workers is that in the early experiments the alkalinity of urine samples that arises on standing caused conversion of some ascorbate to oxalate; if steps are taken to avoid alkalinity then this conversion is minimal (Wandzilak et al. 1994). Recent work (Levine et al. 1996) indicates, however, that both oxalate and urate excretion are elevated beyond a daily intake of $1000 \mathrm{mg}$. (2) Similar anxieties were expressed with regard to urate excretion with the possibility that ascorbate might therefore indirectly exacerbate the effect of urate on gout. Two studies demonstrate that in healthy subjects ascorbate ingestion has no effect on the excretion of urate (Mitch et al. 1981; Schmidt et al. 1981). When high non-physiological plasma levels of ascorbate were induced by continuous infusion of ascorbate in gouty, as compared with normal, subjects (Berger et al. 1977), there was no effect on the clearance of urate indicating that it is highly improbable that high dietary intake of ascorbate has any effect on the urinary excretion of urate in subjects with gout. (3) Low plasma levels of vitamin $\mathbf{B}_{12}$ were reported (Herbert \& Hacob, 1974) to occur in subjects taking large doses of ascorbic acid but this was shown to be explained by analytical error. Erroneously low levels of plasma vitamin $B_{12}$ can occur if no cyanide is added to the assay to liberate protein-bound cobalamins and to stabilize the cobalamins so released (Newmark et al. 1976; Markus et al. 1980). (4) High ascorbic acid intake has been shown to have only a small effect on Fe absorption in healthy Fereplete subjects (see review by Bendich, 1997) which repudiates suggestions that Fe overload could be a consequence of high ascorbate intake (Cook et al. 1984). It appears that the regulation of body Fe stores is unaffected by any increased availability of $\mathrm{Fe}$ from the diet that might be caused by an effect of the excess ascorbate on Fe. (5) Early reports (Cochrane, 1965; Rhead \& Schrauzer, 1971) of rebound scurvy in a small number of subjects following withdrawal of high vitamin C supplements were uncontrolled and have not been substantiated. Studies in guinea-pigs showed no evidence for these claims even when the study was designed to demonstrate a rebound effect. No increased catabolism of ascorbate was demonstrated during high vitamin $C$ dosage nor was there any such increase when the vitamin $\mathrm{C}$ dosage was withdrawn (Norkus \& Rosso, $1975,1981)$. Although the human data available remain contradictory, evidence available at present leads one to conclude that the phenomenon, if it exists at all, does not constitute a significant health problem. (6) Ascorbic acid added to cells in vitro in culture increases the rate of mutagenesis (for review see Rivers, 1989). Detailed reports exist of increased DNA fragmentation, increased DNA repair and chromosome aberrations in cells cultured in media that include added ascorbate. However, these effects only occur in cultures that contain added $\mathrm{Cu}^{2+}$ or $\mathrm{Fe}^{3+}$ ions and when steps were taken to ensure very low levels of these metals in the culture medium, no detrimental effect on DNA was observed. It can be concluded that in any such system in vitro the mutagenic effect of ascorbate is probably due to an ascorbate-metal ion-driven generation of $\mathrm{O}$-derived free radicals. There is no evidence of ascorbate-induced mutagenicity in vivo so that it is highly improbable that any effect that depends on metal ion-driven generation of free radicals caused by ascorbate has any significance. Efficient freeradical scavenging and repair systems protect DNA in vivo from such effects, and intracellular concentrations of ascorbate, and concentrations of metals, which are efficiently sequestered on binding proteins, are so low as to be unlikely to be harmful (Halliwell \& Gutteridge, 1989).

The conclusion from an exhaustive survey of the literature is that oral intake of high (up to $600 \mathrm{mg} / \mathrm{d}$, i.e. six times the current RDA) levels of vitamin $\mathrm{C}$ are safe and entirely free from side-effects (Bendich, 1997). Very high levels (up to $2000 \mathrm{mg} / \mathrm{d}$ ) have not been consistently reported to result in side-effects, although some reports of low reliability suggest that minor side-effects may occur.

\subsection{Vitamin $E$}

There have been four reliable reviews of the toxicological safety of oral intake of vitamin E (Bendich \& Machlin, 1988, 1993; Kappus \& Diplock, 1992; Diplock, 1995). A problem in comparing studies of this nature has been the confusion that exists in the literature between different forms of vitamin E. In particular, IU are often quoted as a measure of quantity. The IU was abandoned by WHO in 1957 (cited by Diplock, 1985) and studies which cite this measure are often ambiguous because it is not possible to determine the precise amount of vitamin $\mathrm{E}$ that was administered. 
The international standard was based on 2-ambo- $\alpha$-tocopheryl acetate, which was an early sample of vitamin $E$ which contained RRR- $\alpha$-tocopheryl acetate with an unknown content of '2-epi- $\alpha$-tocopheryl acetate' (i.e. $S, R, R-\alpha$-tocopheryl acetate). Because of the uncertainty as to the precise composition of this standard, the IU was abandoned, and in 1983 WHO recommended the use of precise descriptions of pure compounds. Despite this, the IU has continued to be used for labelling purposes, particularly in the US and Canada. There is no description of the various stereoisomers present, which may have widely differing biological activity, and this causes serious confusion. Vitamin E activity is expressed in the present paper as $\mathrm{mg}$ RRR- $\alpha$-tocopherol equivalents ( $\alpha$-TE) wherever this is possible. To estimate the total $\alpha$-TE the number of mg of tocopherols present is multiplied by a factor as follows:

$\begin{array}{ll}\text { RRR- } \alpha \text {-tocopherol } & \times 1.0 \\ \text { RRR- } \alpha \text {-tocopheryl acetate } & \times 0.91 \\ \text { RRR- } \alpha \text {-tocopheryl succinate } & \times 0.81 \\ \text { all rac- } \alpha \text {-tocopherol } & \times 0.74 \\ \text { all rac- } \alpha \text {-tocopheryl acetate } & \times 0.67\end{array}$

Study of conventional aspects of the toxicity of vitamin $\mathrm{E}$ in animals was undertaken by many workers over a long period of time (Demole, 1939; Weissberger \& Harris, 1943; Levander et al. 1973; Dysma \& Park, 1975; Krasavage \& Terhaar, 1977; Abdo et al. 1986). There is no evidence of any detrimental effect attributable to vitamin $\mathrm{E}$, and similar conclusions are possible with respect to the teratogenicity and reproductive toxicity of vitamin $\mathrm{E}$ in animals at even large levels of intake of the vitamin (Hook et al. 1974; Krasavage \& Terhaar, 1977). The possibility that vitamin E might have anticlastogenic and mutagenic effects has been studied extensively, and its potential mutagenicity was tested in several different ways (Shamberger et al. 1979; Beckman et al. 1982; Gebhart et al. 1985). Vitamin E was conclusively demonstrated to have no mutagenic properties, and indeed appears to have some effect in reversing the mutagenic effects of other compounds. Although early literature suggested that impure fractions containing vitamin $\mathrm{E}$ had tumour-promoting capability, there is a very large body of evidence that refutes this claim in studies where pure compounds were used (Yang \& Desai, 1977; Weldon et al. 1983). Even at very high levels of inclusion in the feed (up to $25000 \mathrm{IU} / \mathrm{kg}$ feed), vitamin $\mathrm{E}$ was not shown to have any carcinogenic activity.

Many reports purport to deal with the toxicity in human subjects of vitamin $\mathrm{E}$, but there are different levels of reliability that can be attributed to them. Published reports include those that describe single observations on one subject, and planned toxicological studies which may or may not include placebo groups, but which often include bias because they lack blinding. There are fewer highly reliable studies which have been planned and carried out with all necessary rigour, which include sufficient numbers of subjects to enable proper statistical evaluation of the results; they use placebo groups and careful double-blinding, so that the results obtained are valid and reliable. Sufficient such studies have been undertaken to enable an authoritative view of the human toxicity, or lack of toxicity, of vitamin E. There are a few studies (Farrell \& Bieri, 1975; Corrigan, 1982; Ernst \& Matrai, 1985) which do not have adequate controls but are nevertheless of some interest. No consistent adverse effects of vitamin $E$ emerge from these uncontrolled studies. However, the study of Corrigan (1982) indicates that vitamin $\mathrm{E}$ supplementation may aggravate vitamin $\mathrm{K}$ deficiency induced by warfarin anticoagulant therapy (see next paragraph).

With regard to controlled double-blind studies of vitamin E toxicity in man, several reports show conclusively that vitamin $E$ has very low toxicity in human subjects with no consistent adverse effects being reported in most subjects (Anderson \& Reid, 1974; Gillian et al. 1977; Inagaki et al. 1978; Tsai et al. 1978; Stampfer et al. 1983; Bierenbaum et al. 1985; Kitagawa \& Mino, 1989). However, some adverse effects were observed on prothrombin time, or on other factors associated with blood clotting. This question was reviewed carefully (Kappus \& Diplock, 1992; Diplock, 1995); in several studies no effect was observed on blood clotting whereas in other studies there was a marked effect of vitamin $\mathrm{E}$ on some aspects of blood-clotting mechanistics. Following a study of all the reports available it was concluded that vitamin $E$ at a high level of intake may affect the coagulation variables if a low level of vitamin $K$ is also present. Therefore, it is clear that vitamin $\mathrm{E}$ cannot be recommended for administration under these conditions. Vitamin E does not, by itself, cause coagulation abnormalities in persons who have no pre-existing coagulation abnormalities, and in individuals who are the majority of the population, vitamin E supplementation is entirely free from these adverse effects on blood-clotting. This view has been challenged recently (Kim \& White, 1996). In a doubleblind study in twenty-five patients given warfarin therapy, vitamin $\mathrm{E}$ (either 536 or $804 \alpha$-TE/d) was given for 4 weeks without any measurable effects on blood coagulation variables. However, in view of the short time span of this study and the small number of subjects (four per experimental group) it would be unwise to overturn the consensus view that vitamin $\mathrm{E}$ therapy is contra-indicated in subjects with coagulation abnormalities.

The question has been raised (Steinberg, 1993) of the safety of long-term ingestion of amounts of vitamin $E$ in doses of $100 \mathrm{mg} / \mathrm{d}$ which were found (Rimm et al. 1993; Stampfer et al. 1993) to confer significant protection against the risk of coronary artery disease. Higher doses than this (up to about $500 \mathrm{mg}$ vitamin $\mathrm{E}$ daily) have also been recently shown to confer benefit in apparently causing improvement in subjects with angiographically proven cardiovascular disease (Stephens et al. 1996). Results (Takamatsu et al. 1995) of a trial in which $100 \mathrm{mg} \mathrm{d}-\alpha-$ tocopheryl acetate was administered to human subjects for 6 years indicate that there were no adverse effects of the treatment during clinical follow-up. In another study about $250 \mathrm{mg}$ vitamin $\mathrm{E}$ was given daily for 5 years without any side-effects (Greenberg \& The Polyp Prevention Study Group, 1994). While it is not possible at present to state categorically that oral ingestion of large amounts of vitamin $E$ is entirely safe for long periods, because no one has been able to test the toxicity of the vitamin for very long periods of time, application to vitamin $\mathrm{E}$ of the usual criteria of 
safety applied to any drug suggests that not only will longterm supplementation prove to be free from harmful sideeffects but that this may also convey considerable health benefit.

The following conclusions, which were reached earlier (Kappus \& Diplock, 1992; Diplock, 1995) with respect to the safety of oral intake of vitamin $\mathrm{E}$ by human subjects, can be endorsed here. (1) The toxicity of vitamin $E$ is very low. (2) Animal studies show that vitamin $\mathrm{E}$ is not mutagenic, carcinogenic or teratogenic. (3) Reported increases in serum lipids in human subjects following high oral dosage are inconsistent and of little significance. (4) In double-blind human studies, oral dosage resulted in few side-effects, even at a dosage as high as $3 \cdot 2 \mathrm{~g} / \mathrm{d}$. (5) Dosage up to $1000 \mathrm{mg} / \mathrm{d}$ is considered to be entirely safe and without side-effects. (6) Oral intake of high levels of vitamin $E$ can exacerbate the blood coagulation defect of vitamin $\mathrm{K}$ deficiency: high vitamin $\mathrm{E}$ intake is contra-indicated in these subjects.

\subsection{Carotenoids}

Present knowledge of the human toxicology of carotenoids derives almost exclusively from work on $\beta$-carotene; the assumption that other carotenoids have similar toxicology to $\beta$-carotene may not be justified. For instance the absorption, uptake and tissue distribution may differ among the different carotenoids, some of which are bioavailable and others of which are not. Reviews about the safety of $\beta$-carotene (Bendich, 1988; Diplock, 1995) are supported by a review (Wang, 1994) on its absorption and metabolism. Use of $\beta$ carotene as a food and cosmetic colourant and as a drug and nutrient has necessitated extensive reliable toxicity studies done using a range of techniques (Bagdon et al. 1960; Heywood et al. 1985). The Ames test revealed no mutagenicity, which was confirmed by studies using the mouse bone-marrow micronucleus test. Embryotoxicity was not found in rats and rabbits, and in a multiple-generation study in rats given up to $1 \mathrm{~g} / \mathrm{kg}$ per d orally, reproductive function was normal, and there was no interference with embryonic morphology. A study conducted over a 2 -year period in dogs revealed no tumourigenicity or chronic toxicity of any kind and in a mouse carcinogenicity study $\beta$-carotene was without any tumourigenicity. In several organs of dogs and mice given high doses of $\beta$-carotene vacuolated cells were seen due to the formation of fat storage cells; this was not dose-related and was thought to be harmless. These toxicity trials led to $\beta$-carotene being placed in the US Food and Drug Administration category of 'foods generally recognized as safe' for use as a food colourant, in drugs and cosmetics and as a dietary supplement and nutrient (Office of Life Sciences Research, 1979). In addition $\beta$-carotene has been used for 30 years to treat patients with genetically inherited photosensitivities; in this context the ingestion of large amounts of pure $\beta$-carotene has not produced toxic sideeffects (Matthews-Roth, 1986). Some individuals taking supplements of $>30 \mathrm{mg} / \mathrm{d}$ may experience hypercarotenaemia but this disappears quickly after discontinuing the treatment, and it is a benign condition without permanent adverse effects. Anecdotal reports, linked to $\beta$-carotene, of leukopaenia, reproductive disorders, increased prostatic cancer incidence, retinopathy, and allergic reactions have not been substantiated in proper clinical trials. A short-term phase I toxicity trial of supplemental $\beta$-carotene in a small number of human volunteers (Xu et al. 1992) demonstrated a progressive statistically significant decrease in serum vitamin $E$ concentration during supplementation for 9 months with $15,30,45$ and $60 \mathrm{mg} \beta$-carotene/d. However, other studies have demonstrated no such interaction (Willett et al. 1983; Albanes et al. 1992; McLarty, 1992; Goodman et al. 1994; Nierenberg et al. 1994; Ribaya-Mercado et al. 1995). There is no satisfactory explanation available for the results of Xu et al. (1992), but the balance of probability is that there is no likelihood of any interaction between $\beta$ carotene and $\alpha$-tocopherol that would alter the nutritional availability of vitamin $\mathrm{E}$ to human subjects. Careful monitoring of nutrient interactions should become part of all long-term intervention studies.

It can be concluded that supplementation of normal individuals in the population with moderate amounts of $\beta$ carotene can be undertaken safely. The safety of this practice by heavy smokers, who are at high risk of developing lung cancer, has been put in question by two recent studies (ATBC Cancer Prevention Study Group, 1994; Omenn et al. 1996), details of which are given in section 4. The increase in incidence of lung cancer (18\% and $28 \%$ respectively in the two studies) has no easy explanation. Both studies were conducted among subjects who had a high risk of developing lung cancer and may have been at an advanced precancerous state when the $\beta$-carotene administration was commenced. Observational epidemiological evidence suggests that subjects who are not in this state may benefit from $\beta$-carotene administration which is likely to exert a protective role at an early stage of the cancer process. Until further work clarifies the situation in heavy smokers with respect to taking supplements, larger doses should be avoided by such individuals. However, it should be stressed that for normal subjects who do not smoke, $\beta$ carotene supplementation is entirely safe.

\subsection{Non-nutrient antioxidants (flavonoids and other related compounds)}

Few data exist on absorption, metabolism and possible adverse effect of flavonoids and flavonoid-related compounds, such as genistein, daidzein or phenolic acids. Since these compounds are present in our daily diet they are believed to carry no health problems. Obviously, reliable comprehensive safety data, practically non-existent today, will be required, should it appear that increased intakes of specific flavonoids and other phenolic compounds would confer significant health benefits. As concentrations of phenolic compounds in edible plants, fruits and vegetables vary considerably, estimates of human daily intakes range from approximately $100 \mathrm{mg}$ to $1000 \mathrm{mg}$ (Kuhnau, 1976), depending on the diet.

5.5.1. Absorption. It appears that two major metabolic routes operate in man in the absorption of dietary flavonoids. Intestinal micro-organisms hydrolyse the flavonoid glycosides (nearly all the flavonoids are present as glycosides) to their constituent aglycone and sugar. Most of the aglycones are subsequently metabolized by microorganisms. A minor portion is absorbed as aglycones 
(Hackett, 1986). A study on the absorption of quercetin in volunteers found no detectable amount of quercetin or of its metabolites in plasma or in urine (Gugler et al. 1975).

In a more recent study less than $0.25 \%$ of ingested quercetin was found to be excreted, either unchanged or as conjugates, in the urine of healthy ileostomy volunteers (Hollman et al. 1995). In contrast, $0 \cdot 1-1 \cdot 4 \%{ }^{4} \mathrm{C}$-labelled catechin was excreted in urine in the form of unchanged compound. However, $55 \%$ of the ingested dose was excreted as its methylated derivatives (Hackett et al. 1983). There appear to be no human studies available on the absorption and metabolism of anthocyanins.

5.5.2. Possible adverse effects. When tested in vitro in cell cultures, flavonols and especially quercetin have a high cytotoxicity (Babich et al. 1993). However, their toxicity in vivo was shown to be remarkably low when tested in rats (National Toxicological Program, 1992). Most of the flavonols including quercetin are also mutagenic in the Ames test and other short-term tests in vitro (Sugimura et al. 1977; International Agency for Research on Cancer, 1983). However, other tests in vitro have shown quercetin to be a potent inhibitor of mutagenic activity of food carcinogens, such as heterocyclic aromatic amines (Stavric et al. 1990). The same anti-mutagenic activity was found in the livers of mice when quercetin was added to their feed (Stavric, 1994; Stavric et al. 1990). Another test on mice showed the mutagenic effect of heterocylic aromatic amines to be enhanced by quercetin (Rowland, 1993).

Studies on three flavonols, quercetin, myricetin and kaempferol carried out by the molecular toxicology branch of the US Food and Drug Administration point to the prooxidant properties of these compounds and 'suggest a dual role for these flavonoids in mutagenesis and carcinogenesis'. The authors stress that these polyphenolic flavonoids are generally considered to be both antioxidants and anticarcinogens (Sahu, 1992; Sahu \& Gray, 1993, 1994); these studies were performed in isolated rat liver nuclei under aerobic conditions.

A study on quercetin conducted by the US National Toxicological Program (1992) concluded that there was 'some evidence of carcinogenic activity' in male rats receiving $40000 \mathrm{mg}$ quercetin $/ \mathrm{kg}$ diet for 2 years; there was no evidence of carcinogenicity in the lower dose groups. It is important to note that the statistical evaluation of the National Toxicological Program study was not without controversy (Ito, 1992) and a number of previous long-term, well-controlled feeding experiments with rats, mice and hamsters did not show any carcinogenic activity of quercetin (Hirono et al. 1981; Ito et al. 1989). It should also be emphasized that quercetin is one of the most abundant flavonoids, being present in most common vegetables and fruits with an average daily intake estimated to be approximately $25 \mathrm{mg} /$ person (National Toxicological Program, 1992). The consumption of quercetin from a regular diet appears not to induce health problems. The observed very low bioavailability of quercetin in human subjects may explain the apparent contradiction between the results of the tests carried out in vitro and its apparent beneficial effects in man, although the available evidence for beneficial effects of quercetin needs further confirmation. Whereas flavonoids are not mutagenic, anthocyanins have not been tested regarding their potential mutagenic properties. No carcinogenic effects have been reported for other flavonoids.

Tannins, which may exert anticarcinogenic effects by acting as free-radical scavengers, have also been reported to be associated with an increased incidence of oesophageal cancer (Mortin, 1989), but there is no definite evidence to support this assertion. The acute toxicity of tannins was studied in rats, mice and rabbits. Median lethal dose $\left(\mathrm{LD}_{50}\right)$ values for a single dose of orally administered tannins range from 2.25 to $6.00 \mathrm{~g} / \mathrm{kg}$ body weight (Singleton \& Kratzer, 1989). These results are difficult to apply to the human diet since in food the chemical structures and the molecular masses of tannins vary considerably. Anthocyanin extracts are already widely used by the food industry. Anthocyanins obtained by extraction of vegetables and edible fruits have been approved worldwide as food colours (see, for Europe, European Parliament and Council Directive, 1994). They are most commonly based on the following anthocyanidins: peonidin, malvidin, delphinidin, petunidin, pelargonidin, cyanidin. There are two reviews (World Health Organization, 1982; Timberlake, 1988) of the limited toxicological data concerning anthocyanidins. These include data on mutagenicity, reproductive toxicity, and teratogenicity and conclude that anthocyanin-containing extracts are of a very low order of toxicity. The only negative effects were reduced organ and body weights associated with reduced energy intakes at the highest dose, probably reflecting reduced palatability (Clifford, 1996). An average daily intake of $2.5 \mathrm{mg} / \mathrm{kg}$ body weight per $\mathrm{d}$ was allocated for anthocyanin colour from grape skin extracts (World Health Organization, 1982), the composition of which may vary depending on grape variety and extraction process. Anthocyanin colours from grape skin extracts also contain other flavonoids and some tannins. Recently epigallocatechin gallate and green-tea extract have been shown to prevent gastrointestinal carcinogenesis in volunteers; no adverse effects were reported (Yamana et al. 1996).

In conclusion, it is not possible to provide a definitive statement as to the toxicity of flavonoids and other nonnutrient antioxidants, because the literature is either controversial or lacking. A key question that needs to be resolved is the uptake and tissue distribution of non-nutrient substances, which has been hampered by the lack of suitable methodology. Recently a reliable and reproducible method has been published which shows that flavonoids are indeed absorbed in human subjects and enables the measurement in human plasma of flavonoids as their glycosides at concentrations of 0.5-1.6 mmol/l (Paganga \& Rice-Evans, 1997). It seems advisable therefore to test rigorously well-defined flavonoids in toxicological programmes for possible adverse effects if new scientific evidence confirms potential beneficial effects on human health.

\section{Role of food technology in nutritional and safety aspects of antioxidants}

\subsection{Introduction}

Our food supply is safer and offers more variety today than it ever has in the past and this can be largely attributed to the 
applications of food technology to food raw materials. Food processing preserves foods safely so that they can maintain high nutritional and organoleptic values during storage and achieve a wide distribution.

After microbial spoilage, oxidation is the second most important cause of food spoilage, even in those products which might be considered low in oxidizable substrates, such as potato flakes. Important nutrients such as unsaturated lipids, vitamins and proteins can be lost through oxidation. This has been known for a long time and therefore attention to the control of oxidative processes in foods has been one of the highest priorities of the food industry. Until recently antioxidants have been viewed as tools in this fight against oxidation but now, because of growing recognition that antioxidants are themselves important for the maintenance and optimization of health, special efforts may be required to protect the antioxidant nutritional value of food.

$\mathrm{O}_{2}$ is the enemy of antioxidants. This is evident but bears mentioning because the protection of foods from interaction with $\mathrm{O}_{2}$ is the basic principle on which antioxidant protective technologies are based. Many of these have been drawn from experience with lipid oxidation. These technologies can be conveniently broken down into two sections: physical and chemical.

\subsection{Physical processes}

6.2.1. Structural integrity. The structural integrity of the food plays an important role in protecting antioxidants from contact with $\mathrm{O}_{2}$ and thus potential oxidative destruction. In its whole food form, the antioxidant is encased in its protective liposome or cell membrane structures and is out of contact with both $\mathrm{O}_{2}$ and oxygenases. For example, intact oilseeds are quite stable but once they are crushed, extracted and heated in the refining process, resistance of the oils to oxidation decreases. Thus, one nutritional consequence of this loss of structural integrity is negative in that some antioxidants are lost to oxidation. However, the positive nutritional consequence is that antioxidants become more bioavailable.

It has been known for some time that in human subjects the gastrointestinal absorption of carotenoids from vegetables is inversely proportional to particle size (Rodriguez \& Irwin, 1972). Heat treatment also improves absorption: more lycopene appeared in plasma when human subjects drank heated tomato juice compared with the same dose of raw tomato juice (Stahl \& Sies, 1992). In another recent study, ascorbic acid was more bioavailable from cooked broccoli than it was from the raw form (Mangels et al. 1993a). This appears to be a human attribute: studies in other species such as rats (Sweeney \& Marsh, 1974) and preruminant cows (Poor et al. 1993) have shown, respectively, no differences or only small differences in absorbability of cooked $v$. uncooked vegetables. Therefore it may not be appropriate to extrapolate information obtained using animal models to man.

6.2.2. Moisture content. The moisture content of foods should be judiciously chosen and carefully controlled if the antioxidant content of the food is to be optimized since oxidation shows a U-shaped curve, i.e. rapid rates of oxidation when moisture content is low as well as when it becomes too high. Most dehydration processing will destroy lipoxidases but if water content falls below a level which permits the formation of a protective monolayer of water over the surface of the food increased auto-oxidation can result (Labuza, 1971).

According to Labuza (1971), a higher level of water can act as a solvent which mobilizes catalysts and reactants to sites of oxidation. Water may also interact chemically or by $\mathrm{H}$-bonding with other molecular species.

6.2.3. Temperature. Thermal treatment holds an important place in food processing because of the many benefits it brings to food preservation. It also has both a negative and a positive impact on antioxidants. The positive effects include inactivation of oxidases, and breakdown of food structures leading to improved bioavailability. These topics have been dealt with earlier.

Degradation. Thermal treatment also has an important negative impact on antioxidants in foods. Carotenoids and anthocyanins are sensitive to heating: in many studies of foods and food models, these compounds progressively degrade as time and temperature increase. With radical treatments this effect can be radical too. For example at $100^{\circ}$ for $5 \mathrm{~min}$, only $0.93 \% \beta$-carotene was lost in a glycerol model, but $97.8 \%$ was lost when the thermal treatment was carried on for $4 \mathrm{~h}$ at $210^{\circ}$ (Onyewu, 1985). In palm oil thermal destruction of $\beta$-carotene doubles with every $20^{\circ}$ rise in processing temperature (Jideani, 1992). However, technologies exist to protect $\beta$-carotene, tocopherols and tocotrienols. Using a modification of the refining method one Malaysian supplier guarantees specifications of $400 \mathrm{mg}$ $\beta$-carotene $/ \mathrm{kg}$ and $800 \mathrm{mg} / \mathrm{kg}$ minimum of total tocopherols and tocotrienols. This is quite a good level given that most crude palm oils contain on average only $500-750 \mathrm{mg} / \mathrm{kg}$ (Hood, 1995). Water-soluble antioxidants such as ascorbic acid are also sensitive to heating. Blanching of green beans, spinach, broccoli, or peas before freezing resulted in, respectively, losses of $0,20,20$ and $33 \%$ of this vitamin (Unilever, 1995).

Isomerization. Another effect of heating is to increase isomerization of carotenoids in foods. In one study, thermal processing of guava juice increased $c i s$-lycopene levels by 5 -fold and decreased trans-lycopene, although in this food, $\beta$-carotene remained unchanged (Padula \& Rodriguez, 1987). Supplemental $\beta$-carotene added to wholewheat breads and crackers before baking showed significant trans to cis isomerization (4-15\% for bread and 18-23\% for crackers) (Ranhota et al. 1995). More isomerization was observed in the crackers because of the more severe thermal treatment, and less water was left in the final product compared with the bread product. The processing associated with canning is sufficient to cause isomerization of carotenoids: $18-30 \%$ of $\beta$-carotene is converted to cis isomers by usual treatment of typical fruits and vegetables (Quackenbush, 1987). The nutritional significance of this is that the cis isomer of $\beta$-carotene is markedly less well absorbed by the gastrointestinal tract and markedly less well transported in the body (Gaziano et al. 1995). Thus, cis and trans isomers of carotenoids are not biologically equivalent.

Storage temperature. Losses of antioxidants from fresh fruits and vegetables during storage can be quite significant. 
At ambient temperature $90 \%$ of vitamin C was lost from spinach leaves within $3 \mathrm{~d}$. At refrigerator temperatures this loss was greater than $50 \%$. In one recent study, freezing effectively halted any decrease in vitamin $\mathrm{C}$ losses in peas, broccoli, green beans and spinach over 3 months (Unilever, 1995).

6.2.4. Minimizing oxygen. There are some processes which can eliminate or minimize the presence of $\mathrm{O}_{2}$ in the foods. This is quite important as, for example, the rate of degradation of L-ascorbic acid in orange juice (Kennedy $e t$ al. 1992) and in canned, sterilized green beans (Bloeck et al. 1986) depends on $\mathrm{O}_{2}$ present in the sample. Fruit juices containing entrapped air are deaerated by being sprayed into a vacuum deaerator which minimizes the potential for destructive changes to ascorbic acid and other oxidizable components due to $\mathrm{O}_{2}$. Other products e.g. dried red peppers (Lee et al. 1992) can be prepared or packaged under a $\mathrm{N}_{2}$ atmosphere with successful retention of antioxidants.

One of the newer approaches is the potential to use enzymes as antioxidative agents in food systems. Enzymes can act by removing $\mathrm{O}_{2}$, ROS such as $\mathrm{H}_{2} \mathrm{O}_{2}$ and superoxide radicals or by reducing lipid hydroperoxides. The enzymes presently under most study are glucose oxidase $(E C$ 1.1.3.4), thiol oxidase, galactose oxidase ( $E C$ 1.1.3.9), pyranose oxidase and hexose oxidase in conjunction with catalase, superoxide dismutase and glutathione transferase and glutathione peroxidase (for review see Meyer \& Isaksen, 1995). These systems are under study but are not currently of commercial significance in the food industry.

6.2.5. Protection from light. At wavelengths of less than $500 \mathrm{~nm}$, light is an important generator of lipid oxidation and vitamin destruction. Improving packaging by adding colour (red, brown, black) or obscuring material is one of the most effective strategies for protecting foods from light. Some components of foods act as photosensitizers: riboflavin, chlorophyll, myoglobin, haemoglobin pigment, pheophytin and certain conjugated double-bond systems are examples. Sandmeier (1996) has recently shown that curcumin and curcumoid compounds, which are often present in curry mixes, have rather potent photosensitizing properties. Removing these photosensitizers or incorporating food-grade quenchers such as $\beta$-carotene, and $\alpha$ tocopherol, together with chelators of trace metals, can also be effective means of controlling the damaging effects of light.

6.2.6. Irradiation. The effects of irradiation are somewhat variable depending on the food being irradiated, the nutrient under study and the strength and length of time of irradiation. The irradiation of peppers demonstrated no change in vitamin $\mathrm{C}$ content in doses up to $300 \mathrm{~Gy}$ (Mitchell et al. 1992). In fact, post-irradiation storage resulted in increases in total vitamin $\mathrm{C}$ in intact fruits and vegetables (Mitchell et al. 1992).

In one study of chicken meat, $\beta$ - and $\alpha$-tocopherol decreased linearly with irradiation in direct relationship to dose. At $3 \mathrm{kGy}$, which is the maximum recommended by the Food and Drug Administration for chicken, $15 \% \beta$-tocopherol and $30 \% \alpha$-tocopherol were lost (Lakritz \& Thayer, 1992). However, a newer study by the same authors (Lakritz \&
Thayer, 1994) showed different results: only a $6 \%$ reduction in $\alpha$-tocopherol and no significant change for $\beta$-tocopherol in chicken meat at $3 \mathrm{kGy}$ and $2^{\circ}$. Irradiation with a low dose $(0.010 \mathrm{kGy})$ had no effect on the polyphenol content of herb teas (Katusin et al. 1988).

\subsection{Chemical processes}

6.3.1. Enzymes. The control of polyphenol oxidases has been a priority in food science for a long time because oxidized polyphenols are responsible for undesirable brown colours in cut fruits and vegetables, and for the development of off-colours and off-flavours in frozen foods over time. Inactivation of these enzymes can be achieved by rapid high-temperature heat treatment (blanching) while the fresh character of the food can still be retained. Rapid inactivation of the oxidases after pressing apples is a prerequisite for the long-term stability of apple juice (Anonymous, 1993).

Extrusion processes, because of their conditions of high temperature and pressure, destroy oxidases and increase lipid binding to protein and carbohydrate elements in the food which acts to protect from lipid oxidation (Artz et al. 1992).

Cloudy fruit juices are more stable than clear juices because of their content of polyphenols and their glycosides. These latter compounds are not substrates for polyphenol oxidase ( $E C$ 1.10.3.1) (Baruah \& Swain, 1959). For the preparation of clear juices such as apple or raspberry, pectinases are often used. These enzymes hydrolyse the glycosidic side-chains of flavonoids which are then less oxidatively stable in the juice (Rommel \& Wrolstad, $1993 a, b)$. However, glycosides of flavonols and isoflavones are poorly absorbed by the small intestine compared with their aglycones (Brown, 1988). Therefore the nutritional impact of using pectinases is difficult to gauge: on the one hand the polyphenols become more bioavailable, and therefore potentially capable of having an impact on health, but on the other hand there may be fewer of them in the food, as being less stable, they are oxidized.

6.3.2. Supplementation. Supplemental antioxidant nutrients can be added to foods for technological reasons. Ascorbic acid is widely used to increase the resistance to oxidation of many foods. For example, it is used as a dip for cut fruit and vegetables as it is preferentially oxidized instead of the catechol-tannin compounds and effectively inhibits enzymic browning reactions (Potter \& Hotchkiss, 1995). In hydrophobic food matrices, vitamin $\mathrm{E}$ is also frequently used to provide antioxidant protection. Since vitamin $\mathrm{C}$ recycles vitamin $\mathrm{E}$, it is desirable to use both of these antioxidants together. However, since vitamin $\mathrm{C}$ is hydrophilic and vitamin E lipophilic, it is difficult to use them together effectively in food systems. One of the solutions is to use ascorbyl palmitate, a hydrophobic vitamin $\mathrm{C}$ which has been shown to be useful in food lipid systems (Klaui \& Pongracz, 1981).

A mixture of vitamin $\mathrm{E}$, vitamin $\mathrm{C}$ and phospholipids is an effective antioxidant partly because the phospholipid acts as an emulsifier, allowing the two vitamins to be in contact with one another and also because the phospholipid itself actively participates in the antioxidant process (Loeliger et al. 1996). Both vitamins can be incorporated into liposomes 
which can be used as a delivery system in oil-in-water emulsions (Pothakamury \& Barbaosa-Canovas, 1995).

Sometimes higher levels of antioxidants can be incorporated indirectly into a foodstuff. Feeding chickens supplemental vitamin $\mathrm{E}$ increased $\alpha$-tocopherol levels in the meat and this was associated with greater stability (less formation of volatiles) of the meat when it was later irradiated (Patterson \& Stevenson, 1995).

Supplementation with nutrient antioxidants is, thus, a reasonable strategy in many situations for the improvement of the stability and nutritional value of a processed food. Indiscriminate supplementation with antioxidants, as with any nutrient, would however be quite undesirable as there are many unknown factors concerning potential interrelationships in vivo between different antioxidants and eventual long-term effects. To illustrate this point, in a recent study, $\beta$-carotene doses resulted in less lycopene in LDL suggesting that the two related hydrocarbons compete for a similar absorption or transport mechanism (Gaziano et al. 1995).

Non-nutrient antioxidants such as $\mathrm{SO}_{2}$ can be used to advantage to protect those with known or suspected nutritional benefit. For example, the phenolic and procyanidin composition of grape juice processed with $\mathrm{SO}_{2}$ is consistently higher than that of grape juice processed without $\mathrm{SO}_{2}$ addition (Spanos \& Wrolstad, 1990), although there appears to be no effect on quercetin. $\mathrm{SO}_{2}$ will also prevent oxidation of carotenoids but causes bleaching of anthocyanins (Sian \& Soleha, 1991).

Supplemental antioxidants from natural sources such as herbs and spices are effective in conserving foods from oxidation. Many plant extracts have antioxidant activity: rosemary, sage, thyme, oregano, ginger, turmeric, cloves and bay leaves all are active in descending potency (Loliger et al. 1996). Different compounds have very different activities. For example three components of thyme extract, thymol, carvacrol and p-cymene-2,3-diol, were decreasingly resistant to thermal stress in a lard model (Ternes et al. 1995). Mixtures of antioxidants can be obtained from natural sources, for example, by mixing with oil and pressing (Aeschbach \& Wille, 1993), or by using supercritical $\mathrm{CO}_{2}$ extraction of antioxidants. This latter technique has aroused interest because the solvent involved $\left(\mathrm{CO}_{2}\right)$ is nontoxic, non-flammable and of low cost (Tsuda et al. 1995). $\mathrm{O}_{2}$ is almost eliminated from the system. Polyphenols can be recovered through membrane technologies, for example from the waste water from olive oil processing (Trägardh, 1995). It is presently poorly understood whether compounds from this or other fruit and/or vegetable sources have antioxidant ability in vivo similar to that they demonstrate in vitro and therefore could potentially contribute direct health benefits.

\subsection{Conclusions}

The food industry has long experience in the control of oxidative damage in foods and this experience can be used to advantage for the protection of food antioxidants which are beneficial. Some of these, such as vitamins $C$ and $E$ and $\beta$-carotene are known, and strategies for their protection in foods are already exploited by many food technologies.
However, there are many compounds with antioxidant activity which may, or equally may not, have biological activity. Since oxidation cannot be eliminated completely, efforts must be made to identify those antioxidants which are important to health, and in what form they are most useful. This requires that more information must be obtained in the much neglected field of their bioavailability as well as on their bioactivity. With this kind of information, food technology strategies for the preservation of those antioxidants which are beneficial to health could then be applied in a cost-effective manner.

\section{Critical assessment of the science base and conclusions}

\subsection{Identification of criteria}

The foregoing sections have surveyed the science base that underpins the argument that oxidative damage is a significant causative factor in the development of human diseases; that antioxidants are capable of preventing or ameliorating these disease processes; that the administration of antioxidants to human subjects is safe; and that food technology can adapt to meet the needs for nutritional quality and safety in the use of antioxidants in foods. It is now necessary to evaluate clearly what the impact of this survey is on the question of whether an identified specific antioxidant nutrient can be said to positively affect function and have a riskreducing role. This must involve consideration of whether a cause-effect relationship can be established between a dietary antioxidant and a health benefit, and what is the optimum level of intake of the antioxidant in conferring that benefit.

There are four questions the answers to which are considered to be fundamental to providing the required critical assessment.

(1) What is needed to establish that free-radical events are involved in detriment to health which eventually can be associated with the pathology of an identified disease?

(2) What is needed to establish that antioxidants have specific health benefits?

(3) What is needed to make a claim about the functional, nutritional or health benefits of antioxidant nutrients?

(4) What is needed to bring an antioxidant product to the market?

The technology base is very relevant because it is important, in bringing a product to the market, that the active ingredients in the food are still active when the consumer consumes them. A further matter also needs careful consideration. There is a clear difference between, on the one hand optimizing health, by improving intake of food constituents, and on the other hand preventing disease, although the boundaries between these two objectives are not clear-cut. Furthermore, the difference between an effect of a nutrient as a food, and its pharmacological effect, usually at a higher level of intake, needs to be borne in mind. It is important to distinguish between healthy products which optimize well-being and health, and those which may be capable of preventing disease. A single functional food is unlikely by itself to prevent disease; if it were to do so it would be at a very high level of dietary 
intake and questions of toxicity might become important. The disease is the paradigm, the 'experimental model', by means of which it is possible to determine the importance to human subjects of the nutrient. Antioxidants appear to be useful for optimizing well-being, such as in ageing and in simple stress situations, not preventing disease. The future emphasis for functional foods that contain antioxidants must be in providing a suitable level of intake that enhances wellbeing and health. A physician examines a patient by a set of established signs, as a result of which the patient is pronounced 'well'. There is, however, a category beyond this which may be referred to as 'well-being'; this can be defined by objective criteria (McDonald \& Newell, 1996; Spilker, 1996). The optimal level of intake of an antioxidant might be demonstrated to be higher than that which is capable of being delivered by conventional food, in which case the 'functional food' might have the level of the antioxidant enhanced during manufacture by fortification or some other means.

The eventual goal of all this is to determine whether specified foods can be considered as 'functional foods' in that they may be able to confer health benefit following their consumption. For the purposes of this discussion, a functional food is considered to be a food which delivers a physiological benefit, and description of it should convey unambiguous information that is without deception to the consumer about physiological or health benefit. In order that a claim about health benefit can be made, in an ideal world information should be available, which can be critically substantiated from the literature, which establishes the following criteria, each of which, although inter-related with the others, should be individually satisfied.

Criterion 1. A plausible and validated basic science rationale for: (a) the involvement of free radicals in those biochemical and cellular processes which have been shown to lead to detriment to well-being and health, and to specified human diseases; and (b) the involvement of dietary antioxidants in the prevention of these free-radical-involving biochemical processes. This should include results from studies carried out in vitro, in cells in culture, in in vitro/ex vivo models, and in animal models.

Criterion 2. The existence of human population epidemiological data which demonstrate a statistically validated inverse relationship between intake (or preferably serum concentration), of individually specified antioxidants and the risk of, or mortality from, particular diseases.

Criterion 3. The existence of prospective statistically validated epidemiological evidence that links intake (or serum concentration), of identified antioxidants at an early stage of the disease process, with risk of human disease which may develop some time after the exposure to the antioxidants. In such studies both intermediate end-points that have been clearly shown to predict subsequent disease, as well as final end-points, may be used.

Criterion 4. The existence of biomarkers for evaluating free-radical events in human subjects, and the modulating effect on them of antioxidants, which are of interest in maintaining well-being as well as the balance between health and disease. This will include validation of biomarkers, which must preferably be methodologically uncomplicated, by inter-laboratory studies of the same material which has been shown, when investigated in different laboratories, to give the same answers, preferably by more than one method. A further requirement is that the chosen biomarker must be directly relevant, that is it must have functional significance; the biomarker must have been shown by unequivocal techniques to have some significance to both function and the health maintenance or disease risk to which it is linked.

Criterion 5 . The existence of statistically validated interventional human evidence in large groups of human subjects (a) which clearly shows that enhancement of intake of specified antioxidants is associated with improvement in a valid index of well-being and health, or a lowered risk of subsequent disease; (b) which demonstrates optimal levels of intake of antioxidants, derived from indices measured by chosen biomarkers. This would imply that such biomarkers have been established which demonstrate a valid relationship between intake of the antioxidant and the index evaluated by the chosen biomarker(s).

Criterion 6. The existence of clear evidence that the intervention that is proposed with an antioxidant nutrient is safe. This will include evidence that a conclusion as to safety applies with equal force to all groups in the population, including those that are indulging in behaviour which might be expected to increase the risk of the disease concerned.

These are clearly extremely stringent criteria. What is required in coming to a conclusion as to the health benefit of antioxidants is to evaluate the 'state of the art' with respect to them, and to come to a conclusion as to how and to what extent these criteria are satisfied at present.

\subsection{Critical evaluation of the present knowledge base}

Each of the foregoing sections 2-6 has examined in depth one aspect of the science database that exists concerning the discussion here. The following conclusions are derived from those given in detail in the appropriate section.

7.2.1. Conclusions from section 2. An imbalance of ROS and antioxidant defence systems may lead to chemical modifications of biologically relevant macromolecules. This imbalance provides a logical pathobiochemical mechanism for the initiation and development of several disease states. Experimental data obtained in vitro provide evidence that antioxidants function in systems that scavenge ROS and that these are relevant to what occurs in vivo. The relevance in vivo of these observations depends inter alia on knowledge of the uptake and distribution of the antioxidant within the human body, and on what tissue levels of the antioxidant may be expected in relation to dietary levels. Epidemiological studies show a correlation between consumption of foods rich in antioxidants and a decreased risk of several diseases. In particular, diets rich in fruit and vegetables are associated with low risk of several diseases and antioxidants are among the responsible constituents of such a diet. Data on antioxidant supplementation are contradictory and further research is necessary to establish whether supplementation beyond normal dietary intake levels is of benefit.

7.2.2. Conclusions from section 3. There is some way to go until validated precise methods are available for measuring biomarkers of oxidative damage in human 
subjects in vivo under minimally invasive conditions. With respect to oxidative damage to DNA, HPLC and GC-MS methods have both merits and limitations. Oxidation artifacts also arise in sample preparation and in derivatization of semi-purified samples. Lipid oxidation products in plasma are best measured as isoprostanes or as lipid hydroperoxides using specific HPLC techniques. Development of isoprostane measurement will advance specificity and precision and will enable measurement of lipid peroxidation products in urine, which will give a measure of whole-body lipid peroxidation. Measurement of hydrocarbon exhalation is a technique fraught with artifacts and is unsuitable for human studies. The measurement of oxidative damage to proteins has some potential but such methods have not been effectively exploited. Measurement of metabolites derived from RNS has potential, but at present there are many confounding factors which restrict its use.

7.2.3. Conclusions from section 4. Epidemiological studies support the hypothesis that the major antioxidant nutrients vitamin $\mathrm{E}$ and vitamin $\mathrm{C}$, and $\beta$-carotene, which may or may not be acting as an antioxidant in vivo, may play a beneficial role in prevention of several chronic disorders. More research is needed on the impact of other non-nutrient compounds, such as other carotenoids and flavonoids, on human health. In general, human intervention studies using hard end-points are the gold standard. Trials are restricted mainly to the major antioxidants and do not allow firm conclusions because of inconsistent findings, an insufficient number of studies and the use of varying doses. However, there is evidence that large doses of $\beta$-carotene may be deleterious to the health of certain subgroups of the population such as heavy habitual smokers. In functional food research, preventive trials with intermediate end-points may be of help for testing the efficacy of antioxidants. Bioavailability studies and dose-finding studies in combination with the development and application of biomarkers are required for a successful research strategy.

7.2.4. Conclusions from section 5 . Vitamin $\mathrm{C}$ is safe at levels of supplementation up to $600 \mathrm{mg} / \mathrm{d}$, and higher levels, up to $2000 \mathrm{mg} / \mathrm{d}$, are without risk. Vitamin $\mathrm{E}$ has a very low human toxicity and an intake of $1000 \mathrm{mg} / \mathrm{d}$ is without risk; $3200 \mathrm{mg} / \mathrm{d}$ has been shown to be without any consistent risk. Large intakes of $\beta$-carotene must be viewed with caution because they have been shown to confer detriment to a population at high risk of lung cancer when administered after many years of high-risk (smoking) behaviour. Until further work clarifies the situation in heavy smokers with respect to taking supplements, larger doses should be avoided by such individuals. There is little reliable information about the human toxicology of flavonoids and related non-nutrient antioxidant constituents of the diet. A key question is whether these substances are taken up by human subjects and distributed to the tissues in quantities sufficient to confer biological effect.

7.2.5. Conclusions from section 6. The food industry has long experience in the control of oxidative damage in foods and this experience can be used to advantage for the protection of food antioxidants which are beneficial. Some of these, such as vitamins $\mathrm{C}$ and $\mathrm{E}$ and $\beta$-carotene, are well known, and strategies for their protection in foods are already exploited by food technologies. There are, however, many compounds with antioxidant activity which may, or equally may not, have biological activity. Since oxidation during manufacture cannot be eliminated completely, efforts must be made to identify those antioxidants which may be important to health and to discover in what form they are most useful. This requires that more information must be obtained in the much neglected field of their bioavailability as well as of their bioactivity. With this information, food technology strategies for the preservation of those antioxidants which have been shown to be beneficial to health can be applied in a cost-effective manner.

\subsection{Evaluation of criteria}

By using what is revealed in section 7.2 , it is now possible to evaluate the criteria set out in section 7.1.

Criterion 1. There is excellent scientific evidence, derived from several kinds of investigation, for the involvement of free-radical events, either as initiating cause of, or at a later stage in, biochemical and cellular processes that lead to health detriment. Antioxidants that are consumed in the human diet are involved in modulating these free-radicalinvolving events and, thus, they promote health. Valid animal models exist for some forms of cancer and these have been used to demonstrate an apparent preventive effect of some antioxidants against the development of cancer, which gives general support to the contention of health benefit and cancer prevention by antioxidants. There is no acceptable animal model for arteriosclerotic cardiovascular disease. However, there are models which reproduce some aspects of atherosclerosis and limited studies with these support the view that vitamin E may have a role with other factors in delaying the arteriosclerotic process.

Criterion 2. There is human epidemiological evidence for an inverse association between intake of antioxidant nutrients, or the fruits and vegetables that contain them, sometimes supported by measures of serum concentration, and risk of specified diseases in the population. Although there are some instances of lack of such correlations being reported, most studies support this conclusion. The validity of many studies would have been greatly strengthened by inclusion of measures of serum concentrations of the antioxidants under study, and the validity of the conclusion varies in strength for the individual antioxidants under consideration.

Criterion 3. There is abundant prospective epidemiological evidence for a clear correlation between dietary intake of fresh fruit and vegetables and a lowered subsequent risk of cancer. Some data also show a similar correlation with vascular disease. This is a widely accepted view and a claim that increased fruit and vegetable intake is associated with lowered incidence of some forms of cancer and atheromatous cardiovascular disease is entirely valid. Although it is generally thought that this health benefit is conferred by the antioxidant nutrient and non-nutrient substances in fruit and vegetables, no conclusive proof of this contention exists and much confusion has been caused by statements to this effect; data that show an association between high serum levels of specified antioxidants and lowered disease incidence provide no answer to the question 
because the antioxidant may merely be a marker for some active agent derived from fruit and vegetables, or a lifestyle difference associated with high fruit and vegetable intake. The use of 'cancer' or 'cardiovascular disease' as biomarkers of free-radical causation, or antioxidant efficacy, are also unreliable because both diseases are multifactorial and develop over a long period of time with the possibility of multiple inputs into their aetiology, in which antioxidants may be only one relevant factor. There is some evidence, particularly with respect to cardiovascular disease, that links very high intakes of vitamin $E$, derived from food supplements, with a dramatically lowered incidence of disease. However, the likely level of intake in this case is beyond what might be feasibly obtained from the diet, and at normal dietary levels of intake there appears to be little health benefit in this respect. Such an effect could, however, be obtained by the consumption of a functional food fortified to contain a high level of vitamin $E$.

Criterion 4. At the present time biomarkers exist for evaluating oxidative damage to DNA and PUFA; some biomarkers for oxidative damage to proteins also exist. However, there are many anomalies with respect to these biomarkers in that there are both internal inconsistencies in measurement of the same material by different methods, and external inconsistencies in that some laboratories report values that differ widely from those obtained elsewhere using apparently similar methods. There is a great need for validation studies in which methodology is tested within the same laboratory, but also most importantly between different laboratories which must be able to obtain measurements that are consistent with the results of others using the same material. The predictive value of the biomarker in evaluating its relevance to the aetiology of a particular disease also requires validation. Only when this kind of validation has been done and all experts agree as to methodology will it be possible to utilize the methods in a new generation of human studies, which will assess the quantitative relationship between antioxidant intake and health benefit in human subjects.

Criterion 5. The results of intervention studies have been very mixed and at present the situation is confused. There are intervention studies in specified populations from which some conclusions can be drawn. However, it is not valid, and indeed may be highly dangerous, to extrapolate to other populations. For example, intervention studies in China have used end-points which relate to a particularly high natural incidence locally of a particular cancer; the observed data are of interest insofar as they refer to a particular cancer in a specific location. The fact that the incidence of that cancer elsewhere is very low implies that there are other factors involved which may prevent widening the conclusions from China to other locations. Second, the trials involving $\beta$-carotene supplementation in smokers should only be perceived as applying to heavy smokers and must not be applied to other sections of the population who do not smoke or who only smoke moderately. A further disadvantage of present interventional evidence is the fact that, because cost considerations have driven experimental design, individual antioxidants have often not been examined but instead a cocktail of several has been employed. There are no valid studies that demonstrate optimal levels of antioxidant intake in human subjects, and no data exist concerning dose-response relationships of nutrient intake and biological efficacy. Thus, this criterion may prove to be too stringent, although it is important to identify some kind of 'gold standard' in this respect. In order to give informed advice as soon as possible about satisfaction of this criterion it is necessary to shorten the time that is required to conduct and evaluate human studies because those that rely on final end-points are very long and extremely costly to carry out. The development and use of intermediate end-points that give genuine information about the final end-point must, therefore, be an important consideration.

Criterion 6. There is excellent evidence that antioxidant nutrients are safe and can be included in the food of human subjects up to quite high levels without discernible risk. It is necessary to cite some specific cautions, for example, in administering large levels of vitamin $E$ to persons with blood coagulation disorders, and $\beta$-carotene to heavy smokers; such caveats are, however, rare, specific and easily identified. There is less certainty with respect to non-nutrient antioxidants and, if these substances are shown to have potential functional health benefit, then it will be necessary to devote attention to their safety.

\subsection{Final conclusions}

(1) There is evidence that mechanisms that involve free radicals are implicated at some stage of the development of human diseases, and that the maintenance of well-being depends on the supply through the diet of antioxidant nutrients and $\beta$-carotene which modulate free-radical processes in vivo. If it is shown that non-nutrient antioxidants are biologically available then they too may contribute to the total antioxidant effects of the diet in vivo, as well as contributing antioxidant potential during processing, storage and in the gastrointestinal tract.

(2) Present epidemiological evidence is incomplete but in general it supports the basic hypothesis that antioxidant nutrients contribute to well-being and health. There is no evidence to the contrary which would negate this conclusion. There is minimal similar evidence with respect to the non-nutrient antioxidants.

(3) The development of biomarkers for use in human studies is well advanced but biomarkers that are becoming established need critical evaluation before they can be used in a new generation of human studies to examine and evaluate a quantitative cause-and-effect relationship between antioxidant intake and health benefit.

(4) There are few reliable human intervention studies which establish cause-effect relationships, and there is no evidence which shows clearly the optimal amounts of nutrient and non-nutrient antioxidants that are needed in the human diet. The identification and development of intermediate end-points for evaluating the effect of intervention needs further careful work.

(5) Increasing human dietary intake of antioxidants is safe and without undesirable side-effects except in rare welldefined instances.

(6) There is a need to ensure that measures adopted by food technologists in the processing of foods maintain the antioxidant content of the food, and that the antioxidants are 
still active at a suitable level when the food is consumed by the public. If it can be shown with certainty that a certain level of antioxidants in the food is associated with health benefit it may be necessary for food producers to consider means of enhancing the content or form of the antioxidant up to or beyond that usually found in the natural food.

\section{Recommendations for future research}

\subsection{Introduction}

The foregoing section 7 has compared the available scientific database with the evidence that is required to achieve scientific consensus as to what may be needed in order to make a specific claim about the role of antioxidants in maintenance of health and well-being. This enables the identification of areas where further research is required. Improvement in dietary antioxidant intake in human populations is expected to result in lowering of the risk of a number of degenerative diseases. While desirable as an ultimate objective per se, the impact on public health and the resultant decrease in health-care costs make it imperative that substantial sums of money should be spent on research in this important area.

\subsection{Specific recommendations}

Specific recommendations for further research are warranted in each of the areas covered by the foregoing sections 2 to 5 . With regard to section 6 which deals with the role of food technology, there is no particular direction that research needs to take at the present time. Developments in food technology will be based on, and adapt to, nutritional recommendations resulting from biologically driven work. For example, if it is found that the bioactivity of certain flavonoids is high, then steps will have to be taken to preserve, or even enhance, the amounts of these compounds in food products. Similarly, if glycosides of certain compounds prove to be more bioavailable than the free antioxidant then similar considerations will apply to the handling of glycosidic derivatives by food technologists to maximize their content in the food.

8.2.1. Oxidative damage and antioxidant defence systems of the human organism. It is axiomatic to the present considerations that prooxidants cause, or are implicated in the development of, human disease. Direct measurement of prooxidants in vivo is difficult or impossible. It is imperative to establish which are the critical free-radical 'hits' that are the relevant ones; for cancer, which of the multiple modifications of DNA caused by oxidative insult are those that lead to detriment; for atherogenesis, what is the relative relevance of protein and lipid modifications to LDL which contribute to the process, and what is their relationship to the overall multifactorial process of arteriosclerosis? Are the processes examined really relevant to the disease causation? For cancer, there is strong evidence that prooxidants are involved in the initiation stage of the complex process that leads to disease. But what is the relevance of the different forms of oxidative damage to the process? Investigation of this should clarify the role of different antioxidants in controlling carcinogenesis. We need simpler and more specific methods to track ROS both in the initiation and progression of the cancer process. Studies on free-radical involvement in atherosclerosis need to focus more clearly on the relevance of oxidative damage to other factors involved, and on the relative importance of each to the overall complex biochemical process. Noninvasive techniques need to be developed to study these phenomena in human subjects in vivo and this applies particularly to the field of eye diseases which do not readily lend themselves to direct investigation. We need to identify which are the important antioxidants in terms of the maintenance of health, and what is their relationship to one another. We need to clarify whether it is the antioxidant role of the substance that is important, or whether it is some other function, and the possible non-antioxidant effects of antioxidants, in particular with respect to modulation of gene expression, also need further research. We need to know whether there are combined effects of synergy or antagonism both with respect to the antioxidants themselves and to other food components. The primary aim is to identify the active components in the overall system that promotes health. With respect to single antioxidants there is a need for more information regarding regeneration of vitamin $E$ from its radical under cellular conditions, and the role of $\beta$-carotene in apparently exacerbating the cancer process, when given at a late stage to heavy smokers, needs investigation.

8.2.2. Ex vivo methodologies for quantitating and validating damage in vivo to biological macromolecules. Before meaningful work can proceed on providing evidence of the level of antioxidants needed to maintain health and well-being, it is necessary to refine and validate methods that are already available for measurement of oxidative damage in human subjects in a non-invasive manner. This will enable real measurement of variables related to oxidative damage and its attenuation by antioxidants to be made. With respect to biomarkers for oxidative damage to DNA, methods available need to be developed further to prevent oxidation during workup, and formation of artifacts during derivatization of samples, and careful validation studies in a number of different laboratories need to be undertaken using identical test material and methodology. Studies of mitochondrial, as opposed to nuclear, DNA may prove to be of value because there is some evidence that suggests that mitochondrial DNA oxidation correlates with other variables of oxidative damage. Similarly, for biomarkers of lipid oxidation the relatively new isoprostane method needs development and comparison with older established, but less reliable, methods, and this needs to be examined both in plasma and urine as well as other accessible biological material. Validation in many centres by measurement of oxidative damage in the same biological material needs to be undertaken using the same methodology. There is a somewhat longer-term need for development of techniques to be used ex vivo as measures of protein oxidation in vivo, which will, however, probably be able only to reflect the difference at a steady state between damage and repair. It is possible that the present early indications of biomarkers of RNS may be capable of development into useful methodology.

8.2.3. Nutritional options modulating oxidative damage. For proper epidemiological research, as well as for human intervention studies, it would be desirable to put special 
emphasis on the following. (1) Chemical analysis of the antioxidant content of foods so that more realistic food composition tables can be compiled; ideally this should take account of agricultural practices, industrial processing and food preparation. (2) Studies of bioavailability of antioxidants from the diet, and the factors that influence the absorption, distribution and tissue uptake of the compounds and the likely impact of the antioxidants on metabolic processes. This will include studies of the metabolism of antioxidants and the possible metabolic interactions between them. (3) Development and validation of biomarkers of intermediate end-points, both biological response markers and early disease markers, and emphasis on the relevance of the biomarker to the disease end-point as well as the disease process. (4) Application of the validated biomarkers of intermediate end-points in randomized controlled trials testing the efficacy of antioxidants in functional foods for the maintenance of health and wellbeing.

8.2.4. Safety implications of nutritional enhancement of antioxidants. The detailed evidence that is already available which demonstrates that vitamin $\mathrm{C}$ and vitamin $\mathrm{E}$ are safe at quite high levels of inclusion in the human diet, means that it is unnecessary to recommend further work in this area. The safety of $\beta$-carotene was not questioned before the results of the Finnish and American intervention studies, which showed an apparent exacerbation in the incidence of lung cancer in heavy smokers who were given supplements of $\beta$-carotene. This observation needs urgent clarification, and this should also include work on the safety of other carotenoids which have been shown to have biological activity in human subjects. With respect to the flavonoids and other polyphenols, it is likely that their bioactivity will be explored, and the key question of their bioavailability clarified, in the near future. It will be necessary to examine the safety of such bioflavonoid, and other similar compounds, which may be shown to have bioactivity in human subjects, as information becomes available about their bioactivity.

\subsection{Priorities for the recommendations made}

The most urgent requirement for further research is the validation of available biomarkers of oxidative damage. Much useful work has been done already but several anomalies remain and new questions have emerged. Before these biomarkers can be used, in particular as biomarkers of intermediate end-points, in a new generation of human studies, which especially need to assess the level of inclusion of antioxidants in the diet that is optimal for health and well-being, it is necessary to engage in a programme of validation of the biomarkers that are available. These validation studies will also necessitate the inclusion of studies on the analysis of antioxidants and their metabolites. Three types of validation are essential: first there is a need for comparison of results obtained in the same laboratory on identical material using different but complementary methodology so that any numerical differences in the results obtained can be eliminated or at least minimized. Different methods sometimes appear to give different results for what at first sight should be the same measurement by different means. Where numerical differences remain following evaluation, there must be clear reasons given as to the scientific explanation of the difference; for example, the different methods may be measuring slightly different aspects of the same oxidative damage. Second, there is a need for comparison of results obtained in as many different laboratories as possible of identical material which is exchanged between participating laboratories, each of which should use as many agreed methods as possible to assess the degree of oxidative damage in the samples employed. Third, there is a need for a different kind of validation, which is that measurements made must be shown to be clearly linked to those phenomena which give rise to disease in human subjects. For example, is the DNA oxidative damage that is measured a real indicator of the involvement of such damage in mutagenesis and eventual carcinogenicity? Is the oxidation of LDL a reliable indicator of atherogenesis and eventual vascular disease? The development of a disease must be the ultimate paradigm by which the relevance of a biomarker is judged.

As a logical second stage of the work described in the foregoing paragraph, the validated and accepted biomarkers will be used in a new generation of human studies. These need not be as lengthy or time-consuming, and thus not as expensive, as previous prospective epidemiological intervention studies, because the use of intermediate end-points should enable answers to key questions to be obtained considerably more quickly than in earlier studies whose end-points were disease phenomena occurring many years after the initiation of the study. These new studies will provide, for the first time, rigidly controlled evidence of the benefit to be gained from antioxidants in the human diet, and will enable quantitation of the optimal levels of intake of antioxidants. In this connection, care must be taken to ensure that the importance of the antioxidant contribution of the whole diet, as distinct from that of each individual antioxidant, will be evaluated.

The proposals for further research set out in section 8.2 will need to be addressed as and when funds are available, but this should be done at the same time as the major programme of research elaborated in the first two paragraphs of this section. The proposed work set out in the first paragraph could be set in motion with minimal delay and it is anticipated that preliminary results could be available within 2 years of its inception, with reliable final results being available shortly thereafter. The interventional studies in human subjects that are proposed in the second paragraph could commence at the end of 3 years and results would begin to accumulate within 5 years of the initiation of the studies.

Although it is possible to be quite specific with respect to the objectives and proposed methodology of the research that is envisaged, and the time-frame within which specified outcomes may be expected to be achieved, it is beyond the capabilities of those concerned with this report to attempt to evaluate the costs that might be involved. Research costs are a highly volatile quantity and there is considerable variation in cost that might be expected even within different institutions of a single Member State of the European Union. Cost comparisons between different Member States are even more difficult because labour costs, and cost of delivery of 
research, differ very widely. It would be invidious to attempt to identify locations where the research might be carried out more cheaply than in another, and it is the considered view of this group that money identified by the European Union for supporting this research should be made available on the usual competitive basis, with invitation of specific proposals from researchers concerned with their own specific areas of expertise. These can then be assessed both with respect to their scientific merit, as well as their cost, and appropriate awards of money made on this basis, which follows accepted practice in all Member States of the Union.

\section{References}

Abdo KM, Rao G \& Montgomery CA (1986) Thirteen week toxicity study of D-alpha-tocopheryl acetate (vitamin E) in Fischer 344 rats. Food and Chemical Toxicology 24, 10431050.

Aeschbach R \& Wille HJ (1993) Procédé d'extraction d'antioxydant d'origine végétale sous forme liquide (Procedure for extraction of antioxidants of vegetable origin in liquid form). European Patent 639336.

Akasaka K, Ohata A, Ohrui H \& Meguro H (1995) Automatic determination of hydroperoxides of phosphatidycholine and phosphatidylethanolamine in human plasma. Journal of Chromatography B 665, 37-43.

Albanes D \& 22 others (1996) Alpha-tocopherol and beta-carotene supplements and lung cancer incidence in the ATBC cancer prevention study: effects of baseline characteristics and study compliance. Journal of the National Cancer Institute 88, 15601570.

Albanes D, Virtamo J, Rautalahti M, Haukka J, Palmgren J, Gref CG \& Heinonen OP (1992) Serum $\beta$-carotene before and after $\beta$-carotene supplementation. European Journal of Clinical Nutrition 46, 15-24

Ambe KS \& Tappel AL (1961) Oxidative damage to amino acids peptides and proteins by radiation. Journal of Food Science and Technology 26, 448-451.

Ames BN (1989) Endogenous oxidative DNA damage, ageing and cancer. Free Radical Research Communications 7, 121-128.

Anderson TW \& Reid DB (1974) A double-blind trial of vitamin E in angina pectoris. American Journal of Clinical Nutrition 27, 1174-1178.

Anonymous (1993) Production of light colored cloudy juice. Food Marketing and Technology, December issue, 28-32.

Artz WE, Rao SK \& Sauer RM (1992) Lipid oxidation in extruded products during storage as affected by extrusion temperature and selected antioxidants. In Food Extrusion Science and Technology, pp. 55-97 [JL Kokini, CT Ho and MV Karwe, editors]. New York: Marcel Dekker Inc.

ATBC: Alpha Tocopherol, Beta Carotene Cancer Prevention Study Group (1994) The effect of vitamin $E$ and $\beta$-carotene on the incidence of lung cancer and other cancers in male smokers. New England Journal of Medicine 330, 1029-1035.

Azen SP, Qian D, Mack WJ, Sevanlan A, Selzer RH, Liu Ch, Liu Ci \& Hodis HN (1996) Effect of supplementary antioxidant vitamin intake on carotid arterial wall intima-media thickness in a controlled clinical trial of cholesterol lowering. Circulation 94, 2369-2372.

Azzi A, Boscoboinik DO, Marilley D, Ozer NK, Stauble B \& Tasinato A (1995) Vitamin E: a sensor and an information transducer of the cell oxidation state. American Journal of Clinical Nutrition 62, 1337S-1346S.

Babich H, Borenfreund E \& Stern A (1993) Comparative cytotoxicities of selected minor dietary non-nutrients with chemopreventive properties. Cancer Letters 73, 127-133.

Bagdon RE, Zbinden G \& Studer A (1960) Chronic toxicity studies of $\beta$-carotene. Toxicology and Applied Pharmacology 2, 225236.

Baruah P \& Swain T (1959) The action of potato phenolase on flavonoid compounds. Journal of the Science of Food and Agriculture 10, 125.

Beckman JS (1996) Oxidative damage and tyrosine nitration from peroxynitrite. Chemical Research in Toxicology 9, 836-844.

Beckmann C, Roy RM \& Sproule A (1982) Modification of radiation-induced sex-linked recessive lethal mutation frequency by tocopherol. Mutation Research 105, 73-77.

Bendich A (1988) The safety of $\beta$-carotene. Nutrition and Cancer 11, 207-214

Bendich A (1990) Antioxidant nutrients and immune functions introduction. Advances in Experimental Medicine and Biology 262, 1-12.

Bendich A (1997) Vitamin C safety in humans. In Vitamin $C$ in Health and Disease, pp. 367-379 [L Packer and K Fuchs, editors]. New York, NY: Marcel Dekker.

Bendich A \& Langseth L (1995) The effects of vitamin C supplementation: a review. American Journal of Clinical Nutrition 14, 124-136.

Bendich A \& Machlin LJ (1988) Safety of oral intake of vitamin E. American Journal of Clinical Nutrition 48, 642-649.

Bendich A \& Machlin L (1993) The safety of oral intake of vitamin $\mathrm{E}$ : data from clinical studies from 1986 to 1991. In Vitamin $E$ in Health and Disease, pp. 411-417 [L Packer and K Fuchs, editors]. New York, NY: Marcel Dekker.

Bendich A, Machlin LJ, Scandurra O, Burton GW \& Wayner DDM (1986) The antioxidant role of vitamin C. Free Radical Biology and Medicine 2, 419-444.

Berger L, Gerson CD \& Yu TY (1977) The effect of ascorbic acid on uric acid excretion with a commentary on the renal handling of ascorbic acid. American Journal of Medicine 62, 71-76.

Berliner JA \& Heinecke JW (1996) The role of oxidized lipoproteins in atherogenesis. Free Radical Biology and Medicine 20, $707-727$.

Bertram JS \& Bortkiewicz H (1995) Dietary carotenoids inhibit neoplastic transformation and modulate gene expression in mouse and human cells. American Journal of Clinical Nutrition 62, 1322S-1326S.

Bierenbaum ML, Noonan FN, Machlin LJ, Machlin S, Stier A, Watson PB \& Naso AM (1985) The effect of supplemental vitamin $\mathrm{E}$ on serum parameters in diabetics, postcoronary and normal subjects. Nutrition Reports International 31, 11711180 .

Block G, Patterson B \& Subar A (1992) Fruit, vegetables and cancer prevention: a review of the epidemiological evidence. Nutrition and Cancer 18, 1-29.

Bloeck M, Iseli-Winters S, Perren M, Escher F \& Solms J (1986) Quality changes during storage of heat sterilized vegetables. In The Shelf Life of Beverages, pp. 393-411 [G Charalambous, editor]. Amsterdam: Elsevier.

Blot WJ, Li JY, Taylor RR, Guo W, Dawsey S, Wang GQ, Yang CS, Zhrug SF, Gali M, Li GY, Liu BQ, Fangren J, Sum YH, Liu F, Fraument JF, Zhang YH \& Li B (1993) Nutrition intervention trials in Linxian, China. Supplementation with specific vitamin and mineral combinations, cancer incidence and disease-specific mortality in the general population. Journal of the National Cancer Institute 85, 1483-1492.

Brown ED, Morris VM, Rhodes DG, Sinha R \& Levander OA (1995) Urinary excretion of malondialdehyde in subjects fed meat cooked at high or low temperatures. Lipids 30 , 1053-1056.

Brown JP (1988) Hydrolysis of glycosides and esters. In Role of the 
Gut Flora in Toxicity and Cancer, pp. 109-144 [IR Rowlands, editor]. San Diego, CA: Academic Press.

Burk RF \& Ludden TM (1989) Exhaled alkanes as indices of in vivo lipid peroxidation. Biochemistry and Pharmacology 38, 1029-1032.

Cao G \& Cutler RG (1995) Protein oxidation and ageing. Difficulties in measuring reactive protein carbonyls in tissues using 2, 4-dinitrophenylhydrazine. Archives of Biochemistry and Biophysics 320, 106-114.

Cadet J, Ravanat J-L, Buchko GW, Yeo H \& Ames BN (1994) Singlet oxygen DNA damage: chromatographic and mass spectrometric analysis of damage products. Methods in Enzymology 234, 79-88

Chirico S \& Halliwell B (1994) High performance liquid chromatography-based TBA tests. Methods in Enzymology 233, 314-318.

Chu YH \& Lin JY (1993) Factors affecting the content of tocopherol in soyabean oil. Journal of the American Oil Chemists' Society 70, 1263-1268.

Claycamp HG (1992) Phenol sensitization of DNA to subsequent oxidative damage in 8-hydroxydeoxyguanosine assays. Carcinogenesis 13, 1289-1292.

Clifford MN (1996) Anthocyanins in foods. In Proceedings of the Symposium of Polyphenols and Anthocyanins as Food Colorants and Antioxidants, pp. 1-19 [W Phannhauser and A Strigl, editors]. Vienna: University of Vienna.

Cochrane HA (1965) Overnutrition in prenatal life: a problem? Canadian Medical Association Journal 93, 893-899.

Cook JD, Watson SS, Simpson KH, Lipschitz DA \& Skikne BS (1984) The effect of high ascorbic acid supplementation on body iron stores. Blood 64, 721-726.

Corrigan JJ (1982) The effect of vitamin E on warfarin-induced vitamin K deficiency. Annals of the New York Academy of Sciences 393, 361-368.

De Pee S \& West CE (1996) Dietary carotenoids and their role in combating vitamin A deficiency: review of the literature. European Journal of Clinical Nutrition 50, Suppl. 2, 237-259.

Demole V (1939) Pharmakologisches über Vitamin E (Pharmacology of vitamin E). Internationale Zeitschrift für Vitaminforschung 8, 338-341.

Dhanakoti SN \& Draper HH (1987) Response of urinary malondialdehyde to factors that stimulate lipid peroxidation in vivo. Lipids 22, 643-646.

Diplock AT (1985) Fat Soluble Vitamins: Their Biochemistry and Applications. London: Heinemann.

Diplock AT (1994) Antioxidants and disease prevention. In Molecular Aspects of Medicine, vol. 15, pp. 295-376 [H Baum, editor]. Oxford and New York: Pergamon Press.

Diplock AT (1995) Safety of antioxidant vitamins and $\beta$-carotene. American Journal of Clinical Nutrition 62, Suppl. 6, 1510S1516 S.

Dizdaroglu M (1993a) Chemistry of free radical damage to DNA and nucleoproteins. In DNA and Free Radicals, pp. 19-39 [B Halliwell and OJ Aruoma, editors]. Chichester: Ellis Horwood.

Dizdaroglu M (1993b) Quantitative determination of oxidative base damage to DNA by stable isotope-dilution mass spectrometry. FEBS Letters 315, 1-6.

Dysma HA \& Park J (1975) Excess dietary vitamin E in rats. FASEB Journal 34, 912.

Eldin AK \& Appelqvist LA (1996) The chemistry and antioxidant properties of tocopherols and tocotrienols. Lipids 32, 671-701.

El-Sadaani M, Esterbauer H, El-Sayed M, Goher M, Nassar AY \& Jurgens G (1989) A spectrophotometric assay for lipid peroxides in serum lipoproteins using a commercially available reagent. Journal of Lipid Research 30, 627-630.

European Parliament and Council Directive (1994) European Parliament and Council Directive 94/36/EC on colours for use in foodstuffs. L239, 13-29.
Erdman JW, Bierer TL \& Gugger ET (1993) Absorption and transport of carotenoids. Annals of the New York Academy of Sciences 691, 76-85.

Ernst E \& Matrai A (1985) Einfluss von $\alpha$-Tocopherol (Vitamin E) auf die Fliesseigenschaft des Blutes (Influence of $\alpha$-tocopherol (vitamin E) on blood flow characteristics). Therapiewoche 35, 5701-5702.

Esterbauer H, Gebicki J, Puhl H \& Jurgens G (1992) The role of lipid peroxidation and antioxidants in oxidative modification of LDL. Free Radicals in Biology and Medicine 13, 341-390.

Farrell PM \& Bieri JG (1975) Megavitamin E supplementation in man. American Journal of Clinical Nutrition 28, 1381-1386.

Finnegan MTV, Herbert KE, Evans MD, Griffiths HR \& Lunec J (1996) Evidence for sensitization of DNA to oxidative damage during isolation. Free Radicals in Biology and Medicine 20, 9398.

Flagg EW, Coates RJ \& Greenberg RS (1995) Epidemiologic studies of antioxidants and cancer in humans. Journal of the American College of Nutrition 14, 419-426.

Floyd RA, Watson JJ, Wong PK, Altmiller DH \& Rickard RC (1986) Hydroxy-free radical adduct of deoxyguanosine: sensitive detection and mechanisms of formation. Free Radical Research Communications 1, 163-172.

Fridovich I (1986) Superoxide dismutases. Advances in Enzymology 58, 61-97.

Fu S, Gebicki S, Jessup W, Gebicki J \& Dean RT (1995) Biological fate of amino acid, peptide and protein hydroperoxides. Biochemical Journal 311, 821-827.

Gale CR, Martyn CN, Winter PD \& Cooper C (1995) Vitamin C and risk of death from stroke and coronary heart disease in a cohort of elderly people. British Medical Journal 310, 1563-1566.

Garewal H (1995) Antioxidants in oral cancer prevention. American Journal of Clinical Nutrition 62, Suppl. 6, 1410S-1416S.

Gaziano JM, Johnson EJ, Russell RM, Manson JE, Stampfer MJ, Ridker PM, Frei B, Henekens CH \& Krinsky NI (1995) Discrimination in absorption or transport of $\beta$-carotene isomers after oral supplementation with either all-trans- or 9-cis- $\beta$ carotene. American Journal of Clinical Nutrition 61, 1248-1252.

Gebhart E, Wagner H, Grziwok K \& Behnsen M (1985) The actions of anticlastogens in human lymphocyte cultures and their modification by rat liver S9 mix. Chapter II. Studies with vitamins $C$ and E. Mutation Research 149, 83-94

Gerster $H$ (1995) $\beta$-Carotene, vitamin $E$ and vitamin $C$ in different stages of experimental carcinogenesis. European Journal of Clinical Nutrition 49, 155-168.

Gillian RE, Mondell B \& Warbasse JR (1977) Quantitative evaluation of vitamin $E$ in the treatment of angina pectoris. American Heart Journal 93, 444-449.

Giovanucci E, Ascherio A, Rimm EB, Stampfer MJ, Colditz GA \& Willett WC (1995) Intake of carotenoids and retinol in relation to risk of prostate cancer. Journal of the National Cancer Institute 87, 1767-1776.

Giseg SP, Simpson JA, Charlton TS, Duncan MW \& Dean RT (1993) Protein-bound, 3,4-dihydroxyphenylalanine is a major reductant formed during hydroxyl radical damage to proteins. Biochemical Journal 32, 4780-4786.

Giulivi C \& Davies KJA (1993) Dityrosine and tyrosine oxidation products are endogenous markers for the selective proteolysis of oxidatively modified red blood cell hemoglobin by (the 19S) proteasome. Journal of Biological Chemistry 268, 8752-8759.

Goodman GE, Metch BJ \& Omenn GS (1994) The effect of longterm $\beta$-carotene and vitamin $\mathrm{A}$ administration on serum concentrations of $\alpha$-tocopherol. Cancer Epidemiology Biomarkers Prevention 3, 429-432.

Greenberg ER \& The Polyp Prevention Study Group (1994) A clinical trial of antioxidant vitamins to prevent colorectal adenoma. New England Journal of Medicine 331, 141-147. 
Griffiths HR, Lunec J \& Blake DR (1992) Oxygen radical induced fluorescence in proteins: identification of the fluorescent tryptophan metabolite, $N$-formylkynurenine, as a biological index of radical damage. Amino Acids 3, 183-194.

Gugler RM, Leschik M \& Dengler HJ (1975) Disposition of quercetin in man after single oral and intravenous doses. European Journal of Clinical Pharmacology 9, 229-234.

Gutteridge JMC \& Tickner TR (1978) The characterisation of thiobarbituric acid reactivity in human plasma and urine. Analytical Biochemistry 91, 250-257.

Hackett AM (1986) The metabolism of flavonoid compounds in mammals. In Progress in Clinical and Biological Research, vol. 213, pp. 177-194 [VE Cody, JR Middleton and HB Harborne, editors]. New York, NY: A.R. Liss.

Hackett AM, Griffiths LA, Broillet A \& Wermeille M (1983) The metabolism and excretion of $1-\left({ }^{14} \mathrm{C}\right)$-cyanidanol-3 in man following oral administration. Xenobiotica 13, 279-286.

Haddad E, Blankenship JW \& Register UD (1985) Short-term effect of a low-fat diet on plasma retinol and $\alpha$-tocopherol and red cell $\alpha$-tocopherol levels in hyperlipidemic men. American Journal of Clinical Nutrition 41, 599-604.

Halliwell B (1995) Antioxidant characterization; methodology and mechanism. Biochemical Pharmacology 49, 1341-1348.

Halliwell B (1996) Oxidative stress, nutrition and health. Free Radical Research 25, 57-74.

Halliwell B \& Chirico S (1993) Lipid peroxidation: its mechanism, measurement and significance. American Journal of Clinical Nutrition 57, 715S-725S.

Halliwell B \& Dizdaroglu M (1992) The measurement of oxidative damage to DNA by HPLC and GC/MS techniques. Free Radical Research Communications 16, 75-87.

Halliwell B \& Gutteridge JMC (1989) Free Radicals in Biology and Medicine, 2nd ed. Oxford: Clarendon Press.

Hamberg M \& Zhang LY (1995) Quantitative determination of 8hydroxyguanine and guanine by isotope dilution mass spectrometry. Analytical Biochemistry 229, 336-344.

Hanck A (1982) Tolerance and effects of high doses of ascorbic acid. Dosis facit venenum. International Journal of Vitamin and Nutrition Research, Suppl. 23, 221-238.

Harris G, Bashir S \& Winyard PG (1994) 7,8-Dihydro-8-oxo2' deoxyguanosine present in DNA is not simply an artefact of isolation. Carcinogenesis 15, 411-413.

Hennekens CH, Buring JE, Manson JE, Stampfer M, Rosner B, Cook NR, Belanger C, LaMotte F, Gaziano JM, Ridker PM, Willett W \& Peto R (1996) Lack of effect of long-term supplementation with $\beta$-carotene on the incidence of malignant neoplasms and cardiovascular disease. New England Journal of Medicine 334, 1145-1149.

Herbert KE, Evans MD, Finnegan MTV, Griffiths HR \& Lunec J (1996) A novel HPLC procedure for the analysis of 8-oxoguanine in DNA. Free Radicals in Biology and Medicine 3, 467-473.

Herbert V \& Hacob E (1974) Destruction of vitamin $B_{12}$ by ascorbic acid. Journal of the American Medical Association 230, 241-242.

Hertog MGL (1994) Flavonols and flavones in foods and their relation with cancer and coronary heart disease risk. $\mathrm{PhD}$ Thesis, Wageningen University, The Netherlands.

Hertog MGL, Hollman PCH \& van de Putte B (1993) Content of potentially anticarcinogenic flavonoids in tea infusions, wines and fruit juices. Journal of Agricultural and Food Chemistry 41, $1242-1246$.

Heywood R, Palmer AK, Gregson RL \& Hummler H (1985) The toxicity of $\beta$-carotene. Toxicology 36, 91-100.

Hirono I, Ueno I, Hosaka S, Takanashi H, Matshushima T, Sugimura T \& Natori S (1981) Carcinogenicity examination of quercetin and nutin in ACI rats. Cancer Letters 13, 15-21.

Holley AE \& Slater TF (1991) Measurement of lipid hydroperoxides in normal human blood plasma using HPLCchemiluminescence linked to a diode array detector for measuring conjugated dienes. Free Radical Research Communications 15, 51-53.

Hollman PC, de Vries JH, van Leeuwen SD, Mengelers MJ \& Katan MB (1995) Absorption of dietary quercetin glycosides and quercetin in healthy ileostomy volunteers. American Journal of Clinical Nutrition 62, 1276-1282.

Hood RL (1995) Tocotrienols and cholesterol metabolism. In Nutrition, Lipids, Health and Disease, pp. 133-137 [ASH Ong, E Niki and L Packer, editors]. Champaign, IL: AOCS Press.

Hook EB, Healey KM, Niles AM \& Shalko RC (1974) Vitamin E: teratogen or anti-teratogen? Lancet, 809.

Inagaki Y, Kinoshita MO, Nakamura Y \& Masuda YA (1978) Double-blind controlled study of the efficacy of DL- $\alpha$-tocopherylnicotinate in patients with vascular disease. In Tocopherol, Oxygen and Biomembranes, pp. 339-349 [C DeDuve and O Hayashi, editors]. Amsterdam and New York: ElsevierNorth Holland Biomedical Press.

International Agency for Research on Cancer (1983) Quercetin. IARC Monographs on the Evaluation of the Carcinogenic Risk of Chemicals to Humans no. 31, pp. 213-229. Lyon: IARC.

Ito N (1992) Is quercetin carcinogenic? Japanese Journal of Cancer Research 83, 312-313.

Ito N, Hagiwara A, Tamano S, Kagawa M, Shibata M, Kurata Y \& Fukushima $S$ (1989) Lack of carcinogenicity of quercetin in F344/ Du Crj rats. Japanese Journal of Cancer Research 80, 317-325.

Jacob RA \& Burri BJ (1996) Oxidative damage and defense. American Journal of Clinical Nutrition 63, 985S-990S.

Jiang ZY, Woodland ACS \& Wolff SP (1992) Lipid hydroperoxide measurement by oxidation of $\mathrm{Fe}^{2+}$ in the presence of xylenol orange. Comparison with the TBA assay and iodometric method. Lipids 26, 853-857.

Jideani VAE (1992) Carotene retention in palm oil by mechanised and traditional processes. Journal of Food Science and Technology 29, 68-69.

Kallner A, Hartmann D \& Hornig D (1979) Steady-state turnover and body pool of ascorbic acid in man. American Journal of Clinical Nutrition 32, 530-539.

Kanofsky JR (1989) Singlet oxygen production in biological systems. Chemical and Biological Interactions 70, 1-28.

Kappus H \& Diplock AT (1992) Tolerance and safety of vitamin E: a toxicological position report. Free Radicals in Biology and Medicine 13, 55-74.

Karam LS, Bertgold DS \& Simic MG (1991) Biomarkers of OH radical damage in vivo. Free Radical Research Communications 12/13, 11-16.

Katusin RB, Matic S, Razem D \& Mihokovic V (1988) Radiation decontamination of tea herbs. Journal of Food Science and Technology 53, 1120-1126.

Kaugars GE, Silverman S, Lovas JG, Brandt RB, Riley WT, Dao C, Singh VN \& Gallo J (1994) A clinical trial of antioxidant supplements in the treatment of oral leukoplakia. Oral Surgery, Oral Medicine, Oral Pathology 78, 462-468.

Keller J, Halmes NC, Hinson JA \& Humford NR (1993) Immunochemical detection of oxidised proteins. Chemical Research in Toxicology 6, 430-433.

Kennedy JF, Rivera ZS, Lloyd LL, Warner FP \& Jumel K (1992) Ascorbic acid stability in aseptically processed orange juice in TetraBrik cartons and the effect of oxygen. Food Chemistry 45, 327-331.

Kennedy TA \& Liebler DC (1992) Peroxyl radical scavenging by $\beta$-carotene in lipid bilayers. Journal of Biological Chemistry 267, 4658-4663.

Kim JM \& White RH (1996) Effect of vitamin E on the anticoagulant response to warfarin. American Joumal of Cardiology 77, 545-546. 
Kiritsakis A (1990) Chemistry of olive oil. In Olive Oil, pp. 25-55. Champaign, IL: American Oil Chemists Society.

Kitagawa M \& Mino M (1989) Effects of elevated D- $\alpha$-(RRR)tocopherol dosage in man. Journal of Nutritional Science and Vitaminology 35, 133-142.

Klaui H \& Pongracz G (1981) Ascorbic acid and its derivatives as antioxidants in oils and fats. In Vitamin C (Ascorbic Acid), pp. 139-166 [JN Counsell and DH Hornig, editors]. London and New Jersey: Applied Science Publishers.

Kohlmeier L \& Hastings SB (1995) Epidemiologic evidence of a role of carotenoids in cardiovascular disease prevention. American Joumal of Clinical Nutrition 62, 1370S-1376S.

Kondo T (1996) Parkinsons disease and free radicals. Annals of the New York Academy of Sciences 786, 206-216.

Krasavage WJ \& Terhaar CJ (1977) D-alpha-tocopheryl-polyethyleneglycol 1000 succinate. Acute toxicity, subchronic feeding, reproduction and teratologic studies in the rat. Journal of the Science of Food and Agriculture 25, 273-278.

Kritharides L, Jessup W, Gifford J \& Dean RT (1994) A method for defining the stages of oxidation by the separation of cholesterol and cholesterol ester oxidation products using HPLC. Analytical Biochemistry 213, 79-89.

Kuhnau J (1976) The flavonoids. A class of semi-essential food components: their role in human nutrition. World Review of Nutrition and Dietetics 24, 117-191.

Kushi LH, Fulsom AR, Prineas RJ, Mink PJ, Wu Y \& Bostick RM (1996) Dietary antioxidant vitamins and death from coronary heart disease in postmenopausal women. New England Journal of Medicine 334, 1156-1162.

Labuza TP (1971) Kinetics of lipid oxidation in foods. CRC Critical Review of Food Technology, 355-405.

Lachance PA (1996) Future vitamin and antioxidant RDAs for health promotion. Preventive Medicine 25, 46-47.

Lagouri V \& Boskou D (1996) International Journal of Food Sciences and Nutrition 47, 493-497.

Lakritz L \& Thayer DW (1992) Effect of ionising radiation on unesterified tocopherols in fresh chicken breast muscle. Meat Science 32, 257-265.

Lakritz L \& Thayer DW (1994) Effect of gamma radiation on total tocopherols in fresh chicken breast muscle. Meat Science 37, 439-448.

Landrum JT, Bone RA \& Kilburn MD (1997) The macular pigment: a possible role in protection from age-related macular degeneration. Advances in Pharmacology 38, 537-556.

Lee DS, Chung SK \& Yam KL (1992) Carotenoid loss in dried red pepper products. International Journal of Food Science and Technology 27, 179-185.

Levander OA \& Burk RF (1996) Selenium. In Present Knowledge in Nutrition, pp. 320-328 [EE Ziegler and LJ Filer, editors]. Washington, DC: ILSI Press.

Levander OA, Morris VC, Higgs DJ \& Varma RN (1973) Nutritional relationships among vitamin $\mathrm{E}$, selenium, antioxidants and ethyl alcohol in the rat. Journal of Nutrition 103, 536-542.

Levine M, Conry-Cantilena C, Wang Y, Welch RW, Washko PW, Dhariwal KR, Park JB, Lazarev A, Graumlich JF, King J \& Cantilena LR (1996) Vitamin C pharmacokinetics in healthy volunteers: evidence for a recommended dietary allowance. Proceedings of the National Academy of Sciences USA 93, 3704-3709.

Levine RL, Garland D, Oliver CN, Amici A, Climent I, Lenz AG, Ahn BW, Shaltiel S \& Stadtman ER (1995) Determination of carbonyl content in oxidatively modified protein. Methods in Enzymology 186, 464-487.

Levine RL, Williams JA, Stadtman ER \& Shacter E (1994) Carbonyl assay for determination of oxidatively modified proteins. Methods in Enzymology 233, 346-357.

Loeliger J, Lambelet P, Aeschbach R \& Prior E (1996) Natural antioxidants: from radical mechanisms to food stabilisation. In Food Lipids and Health, pp. 68-77 [RE McDonald and DB Min, editors]. New York, NY: Marcel Dekker Inc.

Losonczy KG, Harris TB \& Havlik RJ (1996) Vitamin E and vitamin $\mathrm{C}$ supplement use and risk of all-cause and coronary heart disease mortality in older persons: the Established Populations for Epidemiologic Studies of the Elderly. American Journal of Clinical Nutrition 64, 190-196.

Luis A \& Navab M (1993) Lipoprotein oxidation and gene expression in the artery wall. Biochemical Pharmacology 46, 2119-2126.

Lunec J, Herbert K, Blount S, Podmore ID \& Griffiths HR (1994) 8-Hydroxydeoxyguanosine, marker of oxidative DNA damage in systemic lupus erythematosus. FEBS Letters 348, 131-138.

Lyras L, Shaw PJ, Evans PJ \& Halliwell B (1996) Oxidative damage and motor neurone disease. Difficulties in the measurement of protein carbonyls in human brain tissue. Free Radical Research 24, 399-406.

McDonald I \& Newell C (1996) Measuring Health. A Guide to Rating Scales and Questionnaires, 2nd ed. Oxford: Oxford University Press.

McLarty JW (1992) An intervention trial in high-risk asbestosexposed persons. In The Biology and Prevention of Aerodigestive Tract Cancer, pp. 141-149 [GR Newell and WK Hongs, editors]. New York, NY: Plenum Press Inc.

Malins DC \& Haimonot R (1991) Major alterations in the nucleotide structure of DNA in cancer of the female breast. Cancer Research 51, 5430-5432.

Mangels AR, Block G, Frey CM, Patterson BH, Taylor PR, Norkus EP \& Levander OA (1993a) The bioavailability to humans of ascorbic acid from oranges, orange juice and cooked broccoli is similar to that of synthetic ascorbic acid. Journal of Nutrition 123, 1054-1061.

Mangels AR, Holden JM, Beecher GR, Forman R \& Lanza E (1993b) Carotenoid content of fruits and vegetables: an evaluation of analytic data. Journal of the American Dietetic Association 93, 284-296.

Markus M, Prabhudesai M \& Wassef S (1980) Stability of vitamin $B_{12}$ in the presence of ascorbic acid in food and serum: restoration by cyanide of apparent loss. American Journal of Clinical Nutrition 33, 137-143.

Maskos Z, Rush JD \& Koppenol WH (1992) The hydroxylation of tryptophan. Archives of Biochemistry and Biophysics 296, 514520.

Matthews-Roth MM (1986) $\beta$-Carotene therapy for erythropoietic protoporphyria and other photosensitivity diseases. Biochemie 68, 875-884.

Melia AT, Kosstwardy SG \& Zhi JG (1996) The effect of Orlistat, an inhibitor of dietary fat absorption on the absorption of vitamins $\mathrm{A}$ and $\mathrm{E}$ in healthy volunteers. Journal of Clinical Pharmacology 36, 647-653.

Meyer AS \& Isaksen A (1995) Application of enzymes as food antioxidants. Trends in Food Science and Technology 6, 300304.

Mitch WE, Johnson MW, Kirshenbaum JM \& Lopez RE (1981) Effect of large oral doses of ascorbic acid on uric acid excretion by normal subjects. Clinical Pharmacology and Therapeutics 29, 318-321.

Mitchell GE, McLauchlin RL, Isaacs AR, Williams DJ \& Nottingham SM (1992) Effect of low dose irradiation on composition of tropical fruits and vegetables. Journal of Food Composition and Analysis 5, 291-311.

Mo JY, Maki H \& Sekiguchi M (1992) Hydrolytic elimination of mutagenic nucleotide, 8-oxodGTP, by human 18-kilodalton protein, sanitization of nucleotide pool. Proceedings of the National Academy of Sciences USA 89, 11021-11025.

Morrow JD, Frei B, Longmire AW, Gaziano JM, Lynch SM, 
Shys Y, Strauss WE, Oakes TA \& Roberts LJ (1995) Increase in circulating products of lipid peroxidation ( $F_{2}$ isoprostanes) in smokers. New England Journal of Medicine 332, 1198-1203.

Morrow JD \& Roberts LJ II (1994) Mass spectrometry of prostanoids: $F_{2}$-isoprostanes produced by non-cyclooxygenase free radical-catalyzed mechanism. Methods in Enzymology 233, $163-174$

Morrow JD \& Roberts LJ (1996) The isoprostanes. Biochemical Pharmacology 51,1-9.

Mortin JF (1989) Tannin as carcinogen in bush-tea: tea, mate and khat. In Chemistry and Significance of Condensed Tannins, pp. 403-421 [RW Hemingway and JJ Karchesy, editors]. New York, NY: Plenum Press.

National Toxicological Program (1992) NTP Technical Report on the Toxicology and Carcinogenesis Studies of Quercetin in F344/N Rats. National Institutes of Health Publication no. 91. Washington, DC: National Institutes of Health.

Muldoon M \& Kritchevsky SB (1996) Flavonoids and heart disease. British Medical Journal 312, 458-459.

Newmark HL, Scheiner J, Markus M \& Prabhudesai M (1976) Stability of vitamin $\mathrm{B}_{12}$ in the presence of ascorbic acid. American Journal of Clinical Nutrition 29, 645-649.

Nierenberg DW, Stukel TA, Mott LA \& Greenberg ER (1994) Steady-state serum concentration of alpha tocopherol not altered by supplementation with oral beta carotene. The Polyp Prevention Study 1 Group. Journal of the National Cancer Institute 86, $117-120$.

Niki E (1987) Antioxidants in relation to lipid peroxidation. Chemistry and Physics of Lipids 44, 227-253.

Niki E, Kawakami A, Yamamoto Y \& Kamiya Y (1985) Oxidation of lipids: VIII. Synergistic inhibition of oxidation of phosphatidylcholine liposome in aqueous dispersion by vitamin $\mathrm{E}$ and vitamin C. Bulletin of the Chemical Society of Japan 58, 19711975.

Niki E, Tsuchiya J, Tanimura R \& Kamiya Y (1982) Regeneration of vitamin $\mathrm{E}$ from $\alpha$-chromanoxyl radical by glutathione and vitamin C. Chemistry Letters 27, 789-792.

Norkus EP \& Rosso P (1975) Changes in the ascorbic acid metabolism of the offspring following high maternal intake of this vitamin in the pregnant guinea pig. Annals of the New York Academy of Sciences 258, 401-409.

Norkus EP \& Rosso P (1981) Effects of maternal intake of ascorbic acid on the postnatal metabolism of this vitamin in the guinea pig. Journal of Nutrition 11, 624-630.

Olson JA \& Krinsky NI (1995) Introduction: the colorful fascinating world of carotenoids: important biological modulators. FEBS Letters 9, 1547-1550.

O'Leary V, Darley-Usmar V, Rusell L \& Stone D (1992) Prooxidant effects of lipoxygenase-derived peroxides and antioxidants on the copper-initiated oxidation of low density lipoprotein. Biochemical Journal 282, 631-634.

Office Life Sciences Research (1979) Evaluation of the health aspects of carotene ( $\beta$-carotene) as a food ingredient. Federation of European Biochemistry and Societies' Letters Bethesda Md, USA Contract Number (FDA) 223-75-2004.

Olinski R, Zastawny T, Bubzbon J, Foksincki M, Barecki A \& Dizdaroglu M (1992) DNA base modifications in chromatin of human cancerous tissues. FEBS Letters 309, 193-198.

Oliver CN, Ahn BA \& Moerman EJ (1987) Age related changes in oxidised proteins. Journal of Biological Chemistry 262, 54885491.

Omenn GS, Goodman GE, Thornquist MD, Balmes J, Cullen MR, Glass A, Keogh JP, Meyskens FL, Valanis B, Williams JH, Barnhardt S \& Hammar S (1996) Effects of combination of $\beta$ carotene and vitamin $\mathrm{A}$ on lung cancer and cardiovascular disease. New England Journal of Medicine 334, 1150-1155.

Onyewu PN (1985) Thermal degradation of beta carotene under simulated time and temperature conditions of various food processes. Dissertation Abstracts International B 46, 716.

Oshima H, Freisen M, Brouet I \& Bartsch H (1990) Nitrotyrosine as a new marker for endogenous nitrosation and nitration of proteins. Food and Chemical Toxicology 28, 647-652.

Özer NK, Boscoboinik DO \& Azzi A (1995) New roles of lipoproteins and vitamin $\mathrm{E}$ in the pathogenesis of atherosclerosis. Biochemistry and Molecular Biology International 35, 117-124.

Packer L (1996) Nitric oxide. Part A: sources and detection of NO; NO synthase. Methods in Enzymology 268, 331-340.

Padula M \& Rodriguez ADB (1987) Changes in individual carotenoids and vitamin $\mathrm{C}$ on processing and storage of guava juice. Acta Alimentaria 16, 209-216.

Paganga G \& Rice-Evans CA (1997) The identification of flavonoids as glycosides in human plasma. FEBS Letters 401, 78-82.

Palozza P \& Krinsky NI (1992) Antioxidant effects of carotenoids in vivo and in vitro: an overview. Methods in Enzymology 268, $127-136$

Parker RS (1989) Dietary and biochemical aspects of vitamin E. Advances in Food Nutrition and Research 33, 157-232.

Patterson RLS \& Stevenson MH (1995) Irradiation-induced off odour in chicken and its possible control. British Journal of Poultry Science 36, 425-441.

Poor CL, Bierer TL, Merchen NR, Fahey GC \& Erdman J (1993) The accumulation of $\alpha$ and $\beta$ carotene in serum and tissues of preruminant calves fed raw and steamed carrot slurries. Journal of Nutrition 123, 1296-1304.

Pothakamury UR \& Barbaosa-Canovas GV (1995) Fundamental aspects of controlled release in foods. Trends in Food Science and Technology 6, 397-406.

Potter NN \& Hotchkiss JH (1995) Food Science. New York, NY: Chapman and Hall.

Pryor WA, Squadrito GL \& Friedman M (1995) The cascade mechanism to explain ozone toxicity: the role of lipid ozonation products. Free Radicals in Biology and Medicine 19, 935-941.

Quackenbush FW (1987) Reverse phase HPLC separation of cisand trans-carotenoids and its application to $\beta$-carotenes in food materials. Journal of Liquid Chromatography 10, 643-653.

Ranhota GS, Gelroth J, Langemeier A \& Rogers DE (1995) Stability and contribution of beta carotene added to whole wheat bread and crackers. Cereal Chemistry 72, 139-141.

Ravanat JL, Turesky RJH, Gremaud E, Trudel LJ \& Stadler RH (1995) Determination of 8-oxoguanine in DNA by gas chromatography-mass spectrometry and HPLC-electrochemical detection: overestimation of the background level of the oxidised base by the gas chromatography-mass spectrometry assay. Chemical Research in Toxicology 8, 1039-1045.

Reaven PD \& Witztum JL (1996) Oxidized low density lipoproteins in atherogenesis: role of dietary modification. Annual Reviews in Nutrition 16, 51-71.

Rhead WA \& Schrauzer GN (1971) Risks of long-term ascorbic acid overdosage. Nutrition Reviews 29, 262-263.

Ribaya-Mercado JD, Ordovas JM \& Russell RM (1995) Effect of $\beta$-carotene supplementation on the concentrations and distribution of carotenoids, vitamin $E$, vitamin $A$ and cholesterol in plasma lipoprotein and non-lipoprotein fractions in healthy older women. Journal of the American College of Nutrition 14, 614620.

Rice-Evans C, Diplock AT \& Symons MCR (1991) Techniques in Free Radical Research, pp. 143-147. Amsterdam, London and New York: Elsevier.

Rice-Evans C, Leake D, Bruckdorfer KR \& Diplock AT (1996) Practical approaches to low density lipoprotein oxidation: whys, wherefores and pitfalls. Free Radical Research 25, 285-311.

Rice-Evans C \& Miller NJ (1996) Antioxidant activities of flavonoids as bioactive components of food. Biochemical Society Transactions 24, 790-795. 
Rice-Evans C, Miller NJ, Bolwell PG, Bramley PM \& Pridham JB (1995) The relative antioxidant activity of plant-derived polyphenolic flavonoids. Free Radical Research 22, 375-383.

Rimm EB, Stampfer MJ, Ascherio A, Giovannucci E, Colditz GA \& Willett WC (1993) Vitamin E consumption and the risk of coronary heart disease in men. New England Journal of Medicine 328, 1450-1456.

Rivers JM (1989) Safety of high-level vitamin C ingestion. International Journal of Vitamin and Nutrition Research 30, Suppl., 95-102.

Rock CL, Jacob RA \& Bowen PE (1996) Update on the biological characteristics of the antioxidant micronutrients: vitamin $\mathrm{C}$, vitamin E, and the carotenoids. Journal of the American Dietetics Association 96, 693-702.

Rodriguez MS \& Irwin MI (1972) A conspectus of research on vitamin A requirements in man. Journal of Nutrition 102, 909-968.

Rommel A \& Wrolstad RE (1993a) Ellagic acid content of red raspberry juice as influenced by cultivar, processing and environmental factors. Journal of Agricultural and Food Chemistry 41, 1951-1960.

Rommel A \& Wrolstad RE (1993b) Composition of flavonols in red raspberry juice as influenced by cultivar, processing and environmental factors. Journal of Agricultural and Food Chemistry 41, 1941-1950.

Rowland I (1993) Dietary modification of the activity of food mutagens. Mutagenesis $8,476$.

Sahu SC (1992) Effect of ascorbic acid and curcumin on quercetin induced nuclear DNA damage, lipid peroxidation and protein degradation. Cancer Letters 83, 237-241.

Sahu SC \& Gray GC (1993) Interaction of flavonoids, trace metals and oxygen. Nuclear damage and lipid peroxidation induced by myricetin. Cancer Letters 70, 73-79.

Sahu SC \& Gray GC (1994) Kaempferol-induced nuclear DNA damage and lipid peroxidation. Cancer Letters 85, 159-164.

Sakumi K, Furuichi M, Tsuzuki T \& Kakuma T (1993) Cloning and expression of cDNA for a human enzyme that hydrolyzes 8 oxo-dGTP, a mutagenic substrate for DNA synthesis. Journal of Biological Chemistry 268, 23524-23530.

Sandmeier D (1996) Prooxidative Effekte von Gewürzen unter Lichteinfluss (Prooxidative effects on spices under the influence of light). Fett/Lipid 98, 199-202.

Sarkkinen ES, Uusitupa MI, Nyyssonen K, Parviainen M, Pentitila I \& Salonen J (1993) Effects of two low-fat diets, high and low in polyunsaturated fatty acids, on plasma lipid peroxides and serum vitamin $E$ levels in free-living hypercholesterolaemic men. European Journal of Clinical Nutrition 47, 623-630.

Schalch W (1992) Carotenoids in the retina - a review of their possible role in preventing or limiting change caused by light and oxygen. In Free Radicals and Ageing, pp. 280-289 [I Emerit and B Chance, editors]. Basel: Birkhauser Verlag.

Schmidt KH, Hagmaier V, Hornig DH, Vuilleumier JP \& Rutishauser G (1981) Urinary oxalate excretion after large intakes of ascorbic acid in man. American Journal of Clinical Nutrition 34, 305-311.

Schreck R \& Baeuerle PA (1994) Assessing oxygen radicals as mediators in activation of inducible eukariotic transcription factor NF- $\kappa$ B. Methods in Enzymology 234, 151-163.

Seddon JM, Ajani UA, Sperduto RD, Hiller R, Blair N, Burton TC, Farber MD, Gragoudas ES, Haller J, Miller DT, Yannuuzzi LA \& Willett WC (1994a) Dietary carotenoids, vitamins A, C, and $\mathrm{E}$, and advanced age-related macular degeneration. Journal of the American Medical Association 272, 1413-1420.

Seddon JM, Christen WG, Manson JE, LaMotte FS, Glynn RJ, Buring JE \& Hennekens CH (1994b) The use of vitamin supplements and the risk of cataract among US male physicians. American Journal of Public Health 84, 788-792.
Sen CK \& Packer L (1996) Antioxidant and redox regulation of gene transcription. FEBS Letters 10, 709-720.

Shamberger RJ, Corlett CL, Beaman KD \& Kasten BL (1979) Antioxidants reduce the mutagenic effect of malonaldehyde and $\beta$-propiolactone. Part IX. Antioxidants and cancer. Mutation Research 66, 349-355.

Sharoni Y \& Levi J (1996) Anticarcinogenic properties of lycopene. In Natural Antioxidants and Food Quality in Atherosclerosis and Cancer Prevention, pp. 378-385 [JT Kumpulainen and J Salonen, editors]. Cambridge: Royal Society of Chemistry.

Shigenaga MK, Aboujaoude EN, Chen Q \& Ames BN (1994) Assays of oxidative DNA damage biomarkers. Methods in Enzymology 234, 16-33.

Sian NK \& Soleha I (1991) Carotenoid and anthocyanin contents of papaya and pineapple: influence of blanching and predrying treatments. Food Chemistry 39, 175-185.

Sies H (1991) Oxidative stress: from basic research to clinical application. American Journal of Medicine 91, 31S-39S.

Sies H (1993) Strategies of antioxidant defence. European Journal of Biochemistry 215, 213-219.

Sies H (1997) Antioxidants in Disease Mechanisms and Therapy. London: Academic Press.

Sies H \& Stahl W (1995) Vitamins E and C, $\beta$-carotene, and other carotenoids as antioxidants. American Journal of Clinical Nutrition 62, 1315S-1321S.

Simonian NA \& Coyle JT (1996) Oxidative stress in neurodegenerative diseases. Annual Reviews of Pharmacology and Toxicology 36, 83-106.

Singleton VL \& Kratzer FH (1989) Toxicity and related physiological activity of phenolic substances of plant origin. Journal of Agricultural and Food Chemistry 17, 497-512.

Sokol RJ (1996) Vitamin E. In Present Knowledge in Nutrition, pp. 130-136 [EE Ziegler and LJ Filer, editors]. Washington, DC: ILSI Press.

Spanos GA \& Wrolstad RE (1990) Influence of processing and storage on the phenolic compositions of Thompson seedless grape juice. Journal of Agricultural and Food Chemistry 38, 565-571.

Spilker B (1996) Quality of Life and Pharmacoeconomics in Clinical Trials, 2nd ed. London: Lippincott Raven.

Springfield JR \& Levitt MD (1994) Pitfalls in the use of breath pentane measurements to assess lipid peroxidation. Journal of Lipid Research 35, 1497-1504.

Stahl W \& Sies H (1992) Uptake of lycopene and its geometrical isomers is greater from heat processed than from unprocessed tomato juice in humans. Journal of Nutrition 122, 2161-2166.

Stahl W \& Sies H (1993) Physical quenching of singlet-oxygen and cis-trans isomerization of carotenoids. Annals of the New York Academy of Sciences 691, 10-19.

Stampfer MJ, Hennekens CH, Manson JE, Colditz GA, Rosner B \& Willett WC (1993) Vitamin E consumption and the risk of coronary disease in women. New England Journal of Medicine 328, 1444-1449.

Stampfer MJ, Willett W, Castelli WP, Taylor JO, Fine J \& Hennekens VCH (1983) Effect of vitamin E on lipids. American Journal of Clinical Nutrition 79, 714-716.

Stavric B (1994) Quercetin in our diet: from potent mutagen to probable anticarcinogen. Clinical Biochemistry 27, 245-248.

Stavric B, Mutula TI, Klassen R \& Downie RH (1990) Inhibitory effect of flavonoids against mutagenic and carcinogenic xenobiotics in foods. In Flavonoids in Biology and Medicine. III Current Issues in Flavonoid Research, pp. 515-529 [NP Das, editor]. Singapore: National University of Singapore.

Steinberg D (1993) Antioxidant vitamins and coronary heart disease (editorial; comment). New England Journal of Medicine 328, 1487-1489. 
Steinmetz A \& Potter JD (1991) Review: vegetables, fruit, and cancer. 1. Epidemiology. Cancer Causes and Control 2, 325-357.

Stephens NG, Parsons A, Schofield PM, Kelly F, Cheeseman K \& Mitchinson MJ (1996) Randomised controlled trial of vitamin E in patients with coronary disease: Cambridge Heart Antioxidant Study (CHAOS). Lancet 347, 781-786.

Stillwell WG, Xu HX, Adkins JA, Cramp FT \& Jenski LJ (1989) Analysis of methylated and oxidised purines in urine by capillary gas chromatography-mass spectrometry. Chemical Research in Toxicology 2, 94-99.

Stocker R, Bowry V \& Frei B (1991) Ubiquinol-10 protects human low density lipoprotein more efficiently against lipid peroxidation than does alpha tocopherol. Proceedings of the National Academy of Sciences USA 88, 1646-1650.

Street DA, Comstock GW, Salkeld RM, Schuep W \& Klag MJ (1994) Serum antioxidants and myocardial infarction. Are low levels of carotenoids and $\alpha$-tocopherol risk factors for myocardial infarction? Circulation 90, 1154-1161.

Sugimura T, Nagao M, Matshushima T, Yahagi T, Selno Y, Shiral A, Sawamura M, Natori S, Yoshihira K, Fukuoka M \& Kuroyanagi M (1977) Mutagenicity of flavone derivatives. Proceedings of the Japanese Academy of Science B 853, 194-197.

Sweeney JP \& Marsh AC (1974) Liver storage of vitamin A by rats fed carrots in various forms. Journal of Nutrition 104, 115-120.

Takamatsu S, Takamatsu M, Satoh K, Imaizumi T, Yoshida H, Hiramoto M, Koyama M, Ohgushi Y \& Mizuno S (1995) Effects on health of dietary supplementation with $100 \mathrm{mg} \mathrm{d}$-alphatocopheryl acetate daily for 6 years. Journal of International Medical Research 23, 342-357.

Taylor A (1993) Cataract: relationship between nutrition and oxidation. Journal of the American College of Nutrition 12, 138-146.

Taylor-Mayne S (1996) $\beta$-Carotene, carotenoids, and disease prevention in humans. FASEB Journal 10, 690-701.

Ternes W, Gronemeyer M \& Schwartz K (1995) Determination of pcymene-2,3-diol, thymol, and carvacrol in different foodstuffs. Zeitschrift Lebensmittel Unterschrift Forschungen 201, 544-547.

Thomas SM, Jessup W, Gebicki JM \& Dean RT (1989) A continuous-flow automated assay of iodometric estimation of hydroperoxides. Analytical Biochemistry 176, 353-359.

Timberlake CF (1988) The biological properties of anthocyanins. National Quarterly Information Bulletin, 4-15.

Traber MG \& Sies H (1996) Vitamin E in humans: demand and delivery. Annual Reviews of Nutrition 16, 321-347.

Trägardh G (1995) New developments in membrane processing. In Food Processing: Recent Developments, pp. 231-237 [AG Gaonkar, editor]. Amsterdam: Elsevier Science BV.

Truscott TG (1990) The photophysics and photochemistry of the carotenoids. Journal of Photochemistry and Photobiology 6, 359-371.

Tsai AC, Kelly JJ, Peng B \& Cook N (1978) Study on the effect of megavitamin supplementation in man. American Journal of Clinical Nutrition 31, 831-838.

Tsuda T, Mizuno K \& Ohshima K, Kawakishi S \& Osawa T (1995) Supercritical carbon dioxide extraction of antioxidative components from tamarind (Tamarindus indica $\mathrm{L}$ ) seed coat. Journal of Agricultural and Food Chemistry 43, 2803-2806.

Turk PW \& Weitzman SA (1995) Free radical DNA adduct, 8-hydroxydeoxyguanosine affects activity of HPAII and MSPI restriction endonucleases. Free Radical Research 23, $255-258$.

Uchida K \& Kawakishi S (1993) 2-Oxo-histidine as a novel biological marker for oxidatively modified proteins. FEBS Letters 332, 208-210.

Uchida K \& Kawakishi S (1994) Identification of oxidised histidine generated at the active site of $\mathrm{Cu}, \mathrm{Zn}$-superoxide dismutase exposed to $\mathrm{H}_{2} \mathrm{O}_{2}$. Journal of Biological Chemistry 269, 2405-2410.

Unilever (1995) Diet and Health News, vol. 1. Vlardingen: Unilever Research Laboratories.

van Poppel G \& Goldbohm RA (1995) Epidemiologic evidence for $\beta$-carotene and cancer prevention. American Journal of Clinical Nutrition 62, Suppl., 1393S-1402S.

van Poppel G, Kardinaal A, Princen H \& Kok FJ (1994) Antioxidants and coronary heart disease. Annals of Medicine 26, 429-434.

Velthuis-te Wierik EJ, Van den Berg H, Weststrate JA, Van het Hof KH \& deGraaf C (1996) Consumption of reduced-fat products: effects on parameters of antioxidative capacity. European Journal of Clinical Nutrition 50, 214-219.

Wandzilak TR, D’Andre SD, Davis PA \& Williams HE (1994) Effect of high dose vitamin C on urinary oxalate levels. Journal of Urology 151, 834-837.

Wang XD (1994) Review: absorption and metabolism of $\beta$-carotene. Journal of the American College of Nutrition 13, 314-325.

Wang Z, Ciabattoni G \& Creminon C (1995) Immunological characterization of urinary 8-epi-prostaglandin $F_{2 a}$ excretion in man. Journal of Pharmacology and Experimental Therapeutics 275, 94-100.

Weber P, Bendich A \& Schalch W (1996) Vitamin C and human health - a review of recent data relevant to human requirements. International Journal of Vitamin and Nutrition Research 66, 19-30.

Weissberger LH \& Harris PL (1943) Effect of tocopherols on phosphorus metabolism. Journal of Biological Chemistry 151, 543-551.

Weldon GH, Bhatt A, Keller P \& Hummler H (1983) dl- $\alpha$ Tocopheryl acetate (vitamin E): a long-term toxicity and carcinogenicity study in rats. International Journal of Vitamin and Nutrition Research 53, 287-296.

Wells-Knecht MC, Huggins TG, Dyer DG, Thorpe SR \& Baynes JW (1993) Oxidised amino acids in lens proteins with age. Measurement of o-tyrosine and dityrosine in the ageing human lens. Journal of Biological Chemistry 268, 12348-12352.

Willett WC, Stampfer MJ, Underwood BA, Taylor JO \& Hennekens CH (1983) Vitamins A, E and carotene: effect of supplementation on their plasma levels. American Journal of Clinical Nutrition 38, 559-566.

Wiseman H \& Halliwell B (1996) Damage to DNA by reactive oxygen and nitrogen species: role in inflammatory disease and progression to cancer. Biochemical Journal 313, 17-29.

Wolff SP (1994) Ferrous ion oxidation in the presence of the ferric ion indicator xylenol orange for measurement of hydroperoxides. Methods in Enzymology 233, 182-189.

World Health Organization (1982) Anthocyanins. Toxicological Evaluation of Certain Food Additives. Technical Report Series no. 17 , pp. 42-49. Geneva: WHO.

Xu MJ, Plezia PM, Alberts DS, Emerson SS, Peng YM, Sayers SM, Liu Y, Ritenbaugh C \& Gensler HL (1992) Reduction in plasma or skin $\alpha$-tocopherol concentration with long-term oral administration of $\beta$-carotene in humans and mice. Journal of the National Cancer Institute 84, 1559-1565.

Yamamoto Y (1994) Chemiluminescence-based high performance liquid chromatography assay of lipid hydroperoxides. Methods in Enzymology 233, 319-324.

Yamana T, Nakatani H, Kiknoka B, Matsumoto H, Iwata Y, Kitao Y, Oya K \& Takahashi T (1996) Inhibitory effects and toxicity of green tea polyphenols for gastrointestinal carcinogenesis. Cancer 8, 1662-1667.

Yang NY \& Desai ID (1977) Effect of high levels of dietary vitamin $\mathrm{E}$ on hematological indices and biochemical parameters in rats. Journal of Nutrition 107, 1410-1417. 